

\section{HARVARD UNIVERSITY}

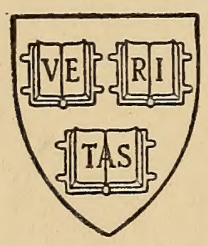

LIBRARY OF THE MUSEUM OF COMPARATIVE ZOÖLOGY GIFT OF

THOMAS BARBOUR 




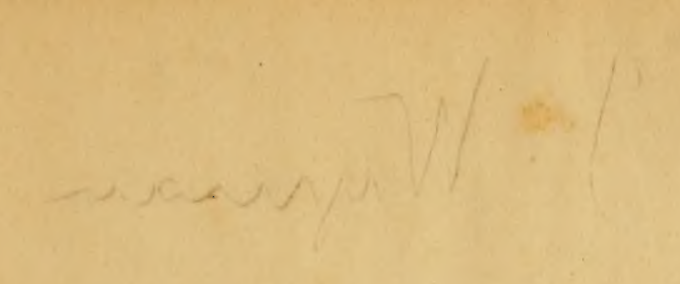




\section{LES ARACHNIDES. $-\infty$}

AVEG UN ATLAS,

PAR M. ANT. DUGÈS,

Professeur à la Faculté de Miédecine de Montpellier, correspondant de l’Acadérnie des Sciences,

ET

PAR M. MILNE EDWARDS,

Membre de I'Institut, professeur au Muséum d'Eistoire Naturelle.

Ku Artas. W 


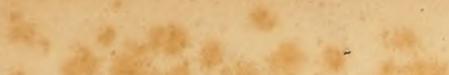

W.

Q

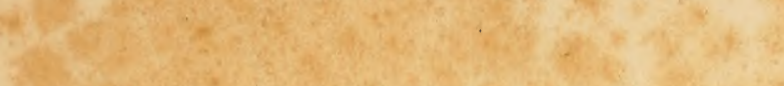

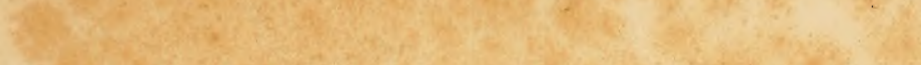

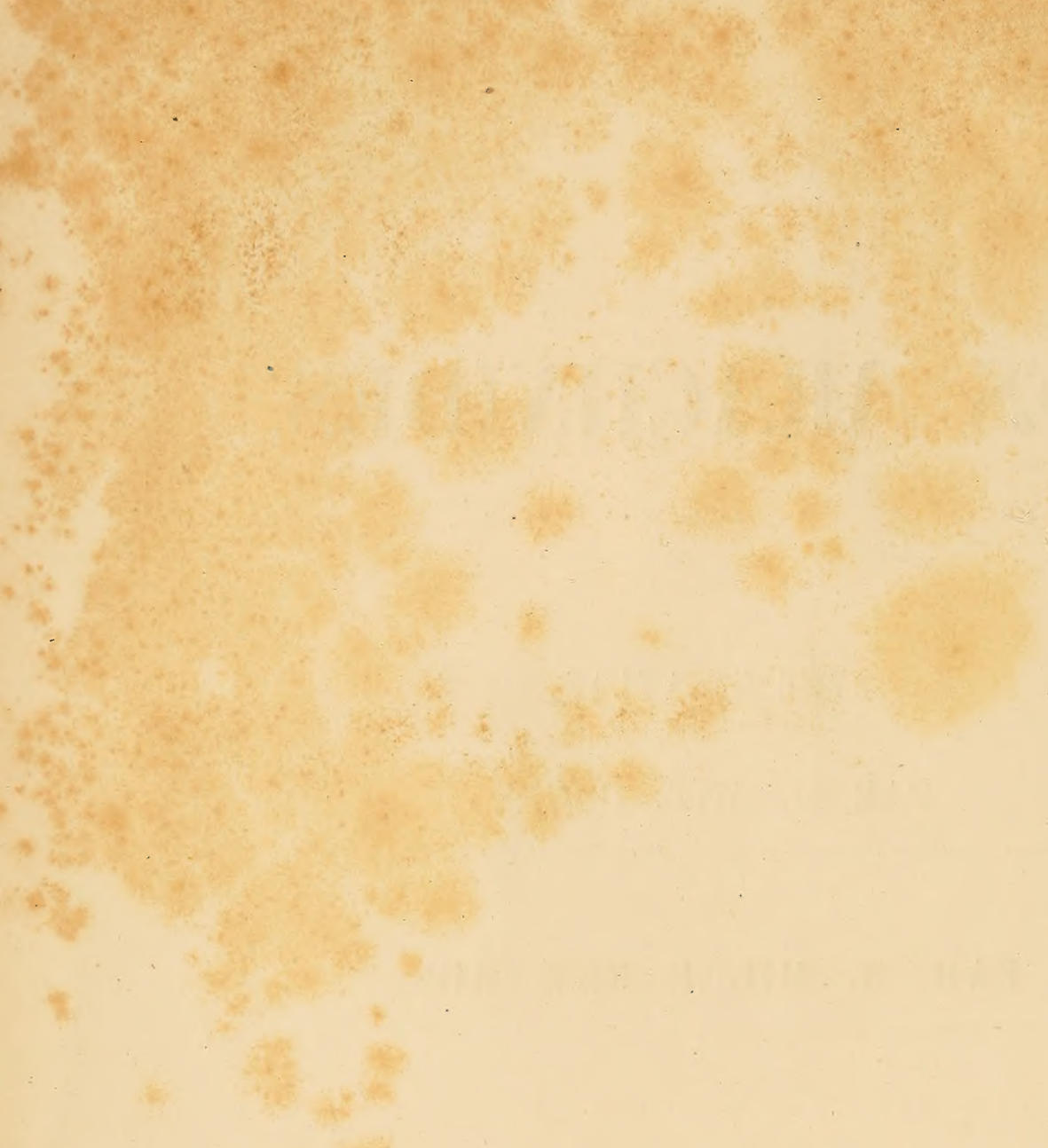

48

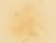

$$
\begin{aligned}
& x^{2}+
\end{aligned}
$$

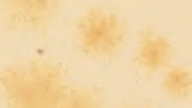

$$
\begin{aligned}
& 3+2 x+2=
\end{aligned}
$$

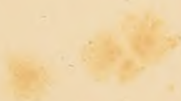


LE

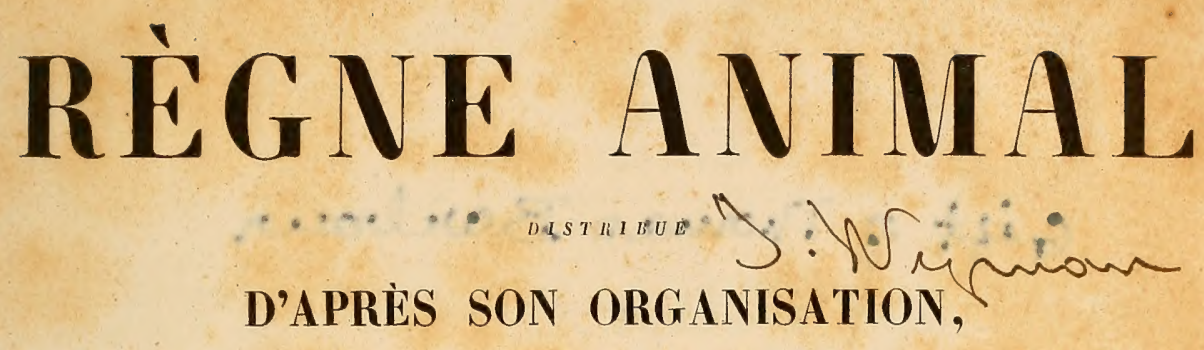

POUR SERVIR DE BASE A L'IIISTOIRE NATURELLE DES ANIMAUX, ET D'intRoduction a L'ANatonie CoMpanée, pAR

\section{GEORGES CUVIER.}

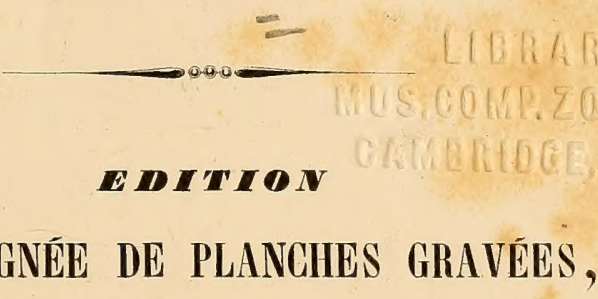

\section{ACCONPAGNÉE DE PLANGHES GRAVÉES,}

LES TYPES DE TOUS LES GENRES,

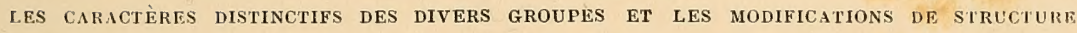
SUR LESQUeLLes REPOSE CETTE ClasSification;

PAR

UIE RÉUNION DE DISCIPLES DE GUVIER,

MM. Audonin, Blanchard, Deshayes, Alcide D'Orbigny, Doyère, Duğès, Duvernoy, Laurillard,

Milne Edwards, Roulin et Valenciennes.

\section{PARIS}

FORTIN, MASSON ET Cie, LIBRAIRES,

PLACE DE L'ÉCOLE-DE-MÉdECINE, N. I.

Imprimé chez Paul Renouard,

Rue Garancière, n. 5. 
Gift of Thomas Barbour

gen of Comper
Zoolagy
APB 5 . 2948

YRAGEIL

rinonogesmog Eum

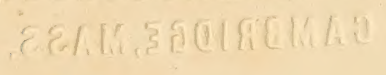





\title{
ARACHNIDES PULMONAIRES.
}

\author{
AN ATOMIE.
}

Fig. 1. Mygale maçonne. Mygale comentaria. Male, pour servir de type à la famille des Aranéides. Grandeur naturelle.

L'aniual est ru par le dos, les pattes étendues, les palpes déployées; entre ces dernièresse voient les mandibules (chelicères ou antennes-pinces de Latreille) attachées au corselet (ou mieux céphaludère), cepkalothorax ou thorax de Latreille. Derrière celui-ci est un abdomen (ou micux thoraeıgastre) pédiculé.

Fig. 1 a. Le corps du même vu en dessous et grossi.

$a$. Mandibules. $b^{t} . l^{\prime}$. Hanches. $c$. Plastron avec la lèvre qui lui est soudée (caractère générique important); huit petites taches brunes répondeut aux attaches de l'entuthorax. $d$. abdomen terrniné par la filière. $e$. Les epercules des poumons ou pneumo-branchies (Latreille) de la paire postérieure. $f$. Sillon où l'on voit i'orifice génital, et qui sépare les opercnles susdites de la plaque destinée à recouvrir les deux poumons antérieurs; des taches brunes sur cette plaque répondent intérieurement à des attaches de muscles.

Fig. 1 b. Parlie antérieure du céphalothorax très grossie et vue de côté.

$a$. La carapace. $b . c$. $d$, Hanches des trois premières paires de pattes du côté ganche. A la première font suite: $x^{\circ}$ le trochanter; $2^{\circ}$ la cuisse, $3^{\circ}$ la jambe, $4_{0}$ le tarse composé de trois articles, dout le dernier armé de griffes. Selon Latreille, le premier article du tarse, armé ici d'uue pointe recourbée, serait une deuxième pièce de la jambe, qui différerait ainsi beaucoup de celle des insectes. II est facile de reconnaître dans les scorpions la vérité de la détermination donseee ci-dessus et qui peut s'appliquer de même aux crustacés. $e$. Le maxille, ou hanche du palpe, quiest composé des mêmes articles que les pattes, mais le dernier modifié en organe copulateur; $f$. crochet de la mandibule.

Fig. I $c$. Longueur des pattes à partir de la ligne médiane du corps.

F zg. $1 d$. Une des deux griffes qui teriminent le tarse, avec l'ergot inséré à leur base.

Fig. 1 e. Le dernier article du palpe très grossi; c'est un des appareils copulateurs les plus simples.

Fig. $1 \mathrm{f}$. L'orifice des organes sécréteurs du sperme, plus grossi que dans la figure $1 a$.

Fig. $1 \mathrm{~g}$. Ces mèmes organes vus en place sur la paroi inférieure de l'abdomen.

Ils n'ont aucune communication avec le palpe, et cependant celui-ci est le seul organe copulateur; son tube corné s'introduit seul dans la vulve de la fornelle; il communique toujours au moius avec une vésicule; mais d'où celle-ci reçit-elle le sperme? Est-il puisé directement dans fe pore génital? Il est certain du moins que ce pore n'est jamais appliqué sur la vulve, comme l'a cru 'Tréviranus; nous l'affirmons positivement pour l'Araignée labyrinthe, le Tétragnathe étendu, et plusieurs autres Aranéides.

Fig. $1 \mathrm{~h}$. Mygale maconne femelle, grandeur naturelle. Comme dans toutes les Aranéides, elle est plus grande et plus trapue que le mâle.

Fig. 1 i. Longueur des pattes.

Fig. $1 k$. Une des griffes avec l'ergot, vue au microscope.

Fig. 1 l. La grifle unique du palpe, qui dilfère par là un peu des pattes et qui manque d'ailleurs de l'avant-dernier arlicle.

Fig. $1 \mathrm{~m}$. Portion de la peau du ventre vue au microseope pour montrer les poils et leur insertion.

Fig. I $n$. Natte musculaire qui double cette peau et dont les faisceaux adhèrent à l'insertion des grands poils; on en voit une au milieu de ce lambeau.

Fig. 1 o. Terrier de la Mygale maconne avec son couvercle; celui du mâle est ordinairement entouré de plusieurs appartenant à des femelles. On le trouve aussi assez souvent vagabond. 


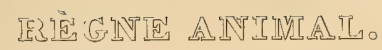
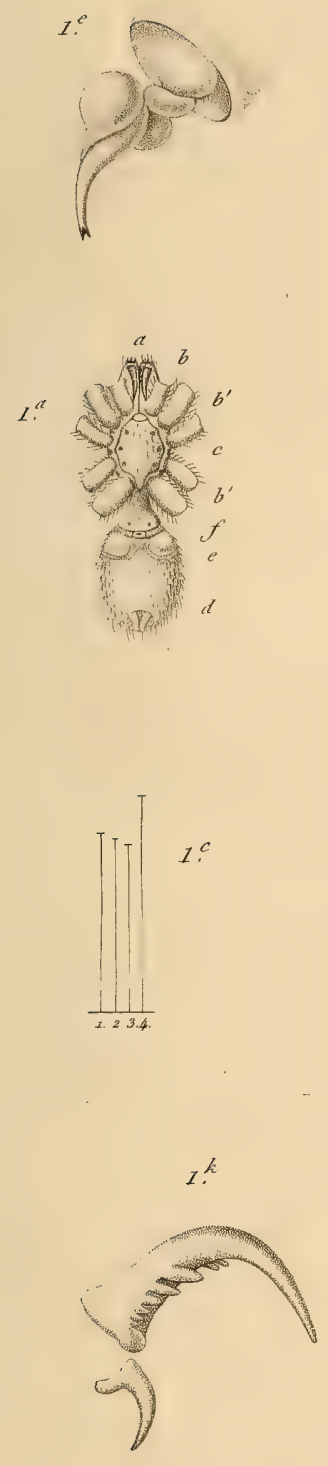

I.

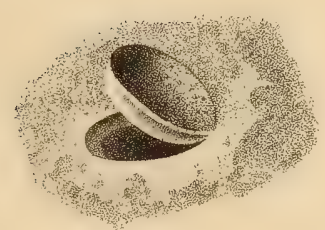

A. Drugies del.
Qrachuides.

Pl. 1.

1.

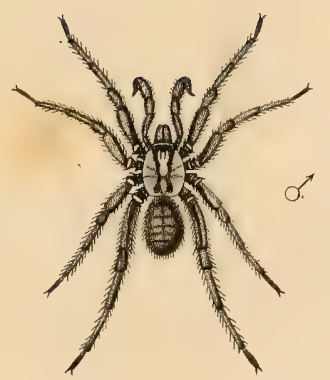

$f^{1}{ }^{d}$
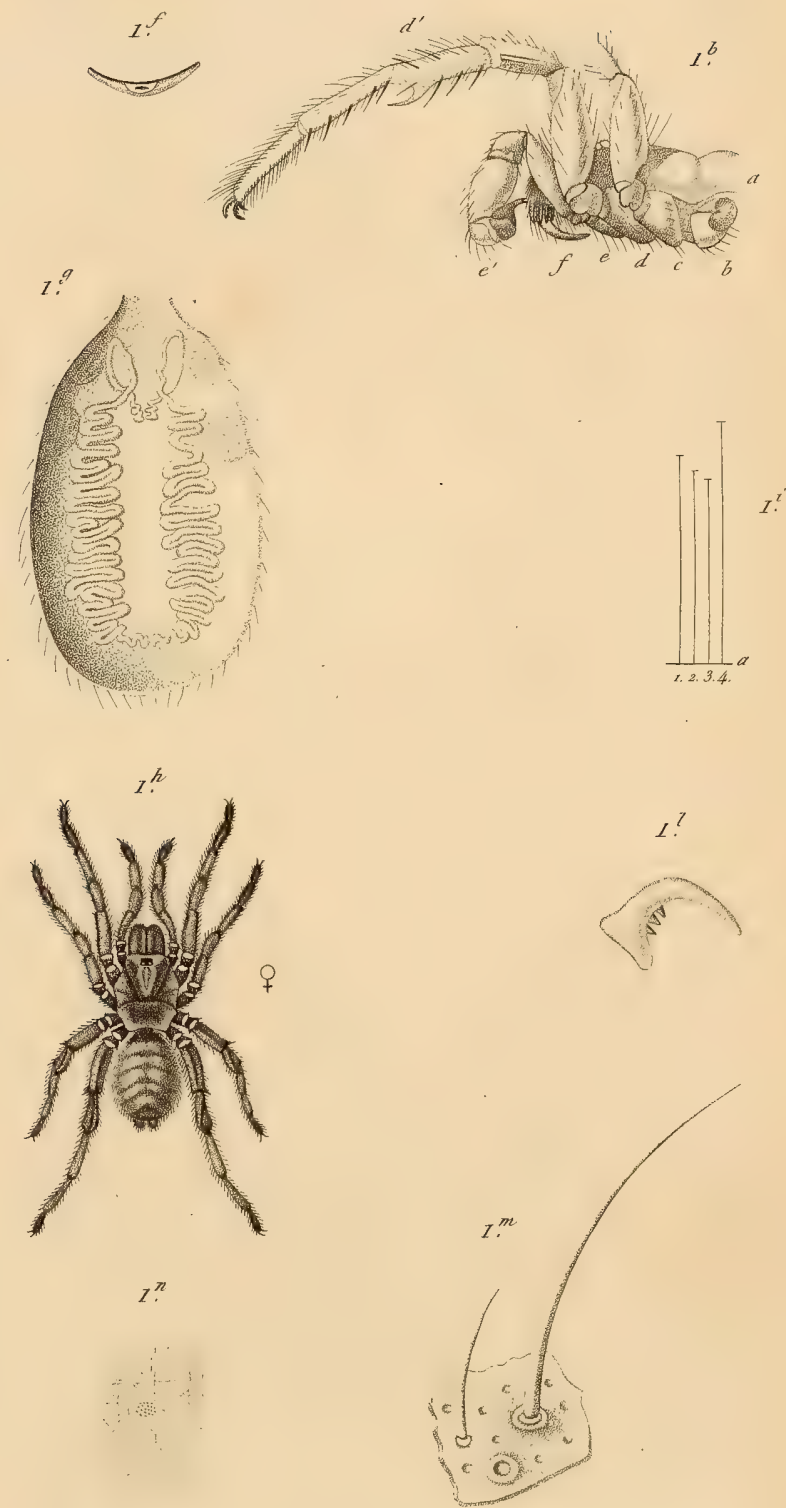

Schmelz so.

1. MYGALE MAÇONNE. (Mygale comentaria. Latr.) 



\title{
ARANÉIDES.
}

\author{
ANATOMIE.
}

Fig. 1. Le devant du céphalothorax de la Mrgale unçonse femelle, très grossi.

a. Le front vu de face, avec l'élévation qui porte les yeux. b. c. Mandibule droite avec ses poils raides et son râteau. $\not$. La gauche, dépouillée des uns et des autres. $e$. Son crochet vu en raccourci.

Fig. 2. Les yeux médians vus au microscope sur une coupe perpendiculaire du front.

En $a$. le cristallin est en place dans la cornée, et séparé de la chorö̈de é de Ja rétine; en $b$. la cornée est isolée; Je cristallin $c$. est enveloppé d'une capule molle représentant à-la-fois la choroïde, la rétine et le corps vitré; le nerf optique s'y perd en s'élargissant.

Fig.3. L'éminence oculifère vue perpendiculairement, plus grossie que dans la figure 1 et exprimant mieux la différence des axes de chacun des yeux.

Fig. 4. Mandibule gauche vue par la face interne.

$a$. le corps. $b$. le crochet au dessus duquel se voient 7 dents qui bordent en dedan 3 la gouttière où il se loge.

Fig. 5. Une des dents du râteau qui est vu de profil dans la figure précédente, et de face dans la figure 1.

Fig. 6. Le crochet mandibulaire très grossi, portant la glande venimeuse. Elle est ici fort petite, et logée dans la mandibule. La plupart des autres Aranéides l'ont grande et logée dans le corselet; mais on ne doit pas croire que leur piqùre puisse êtrepour cela bearcoup plus dangereuse, encore moins qu'elle ait jamais été mortelle pour l'homme.

Fig. 7. Extrémité de ce crochet beaucoup plus grossie et vue de face pour montrer la fente et le trou vénénifères.

Fig. 8. Vue anatomique de la face inférieure du corps de la même Mygale, le plastron largement perforé, et une partie de la peau de l'abdomen renversée en dehors.

a. Mandibules (ou chélicères. Latr.). - - b. Maxille offrant un creux bordé de dents en arcade, au voisinage de la lèrre. - c.c. Hanches. Entre elles se voit le ganglion nerveux principal, ses branches et son double cordon postérieur, terminé par un autre renflement ganglionnaire. Un des nombreux filets donnés par celui-ci va jusqu'aux filières, en côtoyant l'ovaire qui le cache en partie. $-d . d$ '. Plaques operculaires des poumons avec leur stigmate. Sur la plaque antérieure et au devant d'elle, est de chaque côté un faux stigmate répondant à des attaclies de muscles. - $e$. Le poumon antérieur gaucbe renversé avec la peau à laquelle il adhère près du stigmate, par le moyen d'une lame cornée qui soutient les feuillets membraneux. Ceux-ci ont été figurés en petit nombre pour éviter la confusion. $-e^{\prime}$. Le poumon postérienr gauche masqué par un grand muscle $f$. et par le pannicule charnu de l'abdomen (Voy, pl I. fig. I $n_{\text {. }}$ ). g. Sillon dans lequel est la vulve. A l'un des angles on voit s'insérer l'oviducte; l'ovaire gauche lui fait suite; ses ovules se détachent bien des granulations du foie qui remplit tout l'abdomen, et de l'organe sécréteur de la soie visible au voisinage des filières, _h filière et anus bilabiè.

(D'après nature.) 


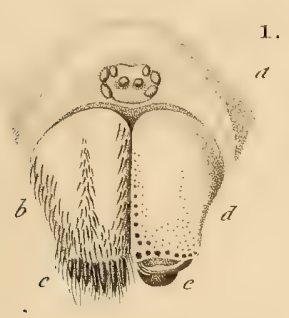

8.
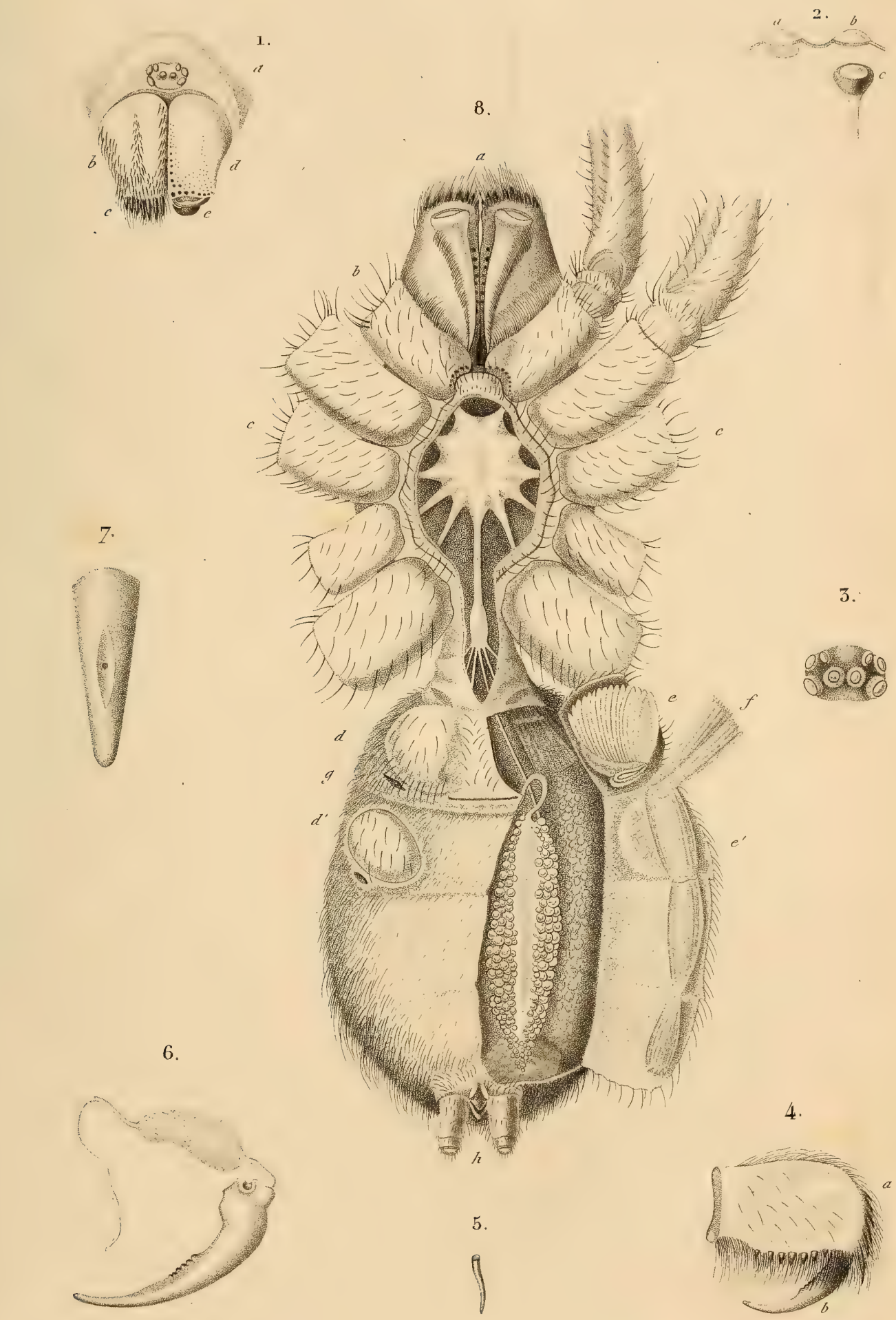

5.

9

4.

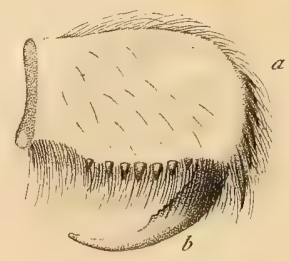

Schtnelz so. 






\title{
ARANÉIDES.
}

\author{
ANATOMIE.
}

Fig. 1. Corselet de la Mrgale vu du côté gauche et considérablement grossi.

$a$. Mandibule. - b. Carapace. - $c$. Les yeux. - $d$. Coupe du plastron sur la ligne médiane. - e. Coupe de la lèrre (languette de Latreille) qui est soudée an plastron. - $f_{0}$ Camérostome de Latreille (chaperon?) mis à découvert par l'ablation de la maxille. gauche. - g. Ouverture et cavité de la bouche, bornée en avant par ce que Latreille nomme le palais (labre?), et en arrière par la langue que Latreille $n^{3} a$ pudistinguer de la lèvre antérieure; elle est iei détachée du palais et renversée en dehors en trois lambeanx. - $h$. L'œsophage corné en dessus, membraneux en dessous, traversant le collier nervenx. - $i$. Pièce cornée à la paroi supérieure de l'estomac. - $j$. Ganglion cérébral ou sułœsophagien, qui donne de claque côté un nerf récurrent ou splanchnique, quatre nerfs optiques $k$, un nerf mandibulaire $l .-m$. Ganglion principal (Voyez pl. II. fig. 8.). - n. Son cordon terminal.

Fig. 2. L'ouverture de la bouche vue de face.

$a$. Camérostome surmonté d'une sorte de luette. $-b$. La lèvre extérieure séparée du camérostome par la fente buccale.

Fig. 3. Le palais et la langue séparés et renversés, l'un en avant, l'autre en arrière, pièces cornéo-membraneuses, canaliculées, réunies latéralement par une membranule dont on voit ici quelques restes, emboîtées l'une par l'autre, la première convexe etla deuxième concave, mues par des muscles assez nombreux, articulées en arrière avec un élargissement et une bifurcation de l'œesophage qui lui-mème s'articule avec la pièce cornée de l'estomac. Cet ensemble tombe avec la dépouille extérieure et y reste adhérent dans la mue.

Fig. 4. Pièce cornée de l'estomac vue en dessous.

Fig. 5. Coupe transversale de la même.

Frg. 6. Organes digestifs vus par en haut; grossissement moindre que dans les figures précédentes; un trait ponctué indique la circonscription de l'abdomen.

$a$. Ganglion cérébral. - $b$. Tète bifurquée de l'œsophage. $-c$. L'estomac avec les cœenms latéraux qui pénètrent dans les palpes et les pattes jusqu'au bout de la cuisse. $-d$. Partie qui traverse le pédicule de l'abdomen. - e. Elargissement (dnodenum) qui reçoit le gros trono des vaisseanx biliaires; les sucs alimentaires y pénètrent sans doute, car l'abdomen grossit énormément quand une proie très volumineuse arrive après un long jeûne. - $f$. Intestin grèle. - $g$. Gros intestin ou cœeum d'où part en arrière le rectum, et en avant les deux canaux urinaires $h$. qui se ramifient a l'infini entre les granulations du foie.

Fig. 7. Portion abdominale du tube digestif vue de profil; les mêmes lettres expriment les mêmes choses que dans la figure précédente.

Fig. 8. Entothorax (enthodère selon nous) vu en dessous ; grandeur proportionnelle à celle de la figure 6 . On distingue : $1^{\circ}$ les huit branches fibro-cartilagineuses qui embrassent le ganglion principal et le fixent au plastron ; $2^{\circ}$ la lame principale dont la concavité tournée en haut recoit l'estomac.

Fig. 9. Quelques-unes des vésicules cœcales ou granulations du foie, et entre elles les filamens des canaux urinaires.

Fig. 10. Portion d'un de ces filamens sonmise au microscope; elle contient des globules beaucoup plus gros que ceux du sang.

Fig. 11. Coeur de la Mygale maçonne vu en dessus; un trait ponctué indique le contour de l'abdomen.

$a$. Prolongement qui va dans le corselet. $-b$. Vaisseaux du poumon antérieur? -c. Vaisseaux du poumon postérieur?

Fig. 12. Un des renflemens du cœur plus grossi, pour mieux faire voir les fibres transversales et longitudinales (médianes).

Fig. 13. Le mème dont on a enlevé la paroi supérieure pour montrer sa cavité prismatique et les plis valvulaires qui cachent l'origine des vaisseaux.

(D'aprés nature.) 


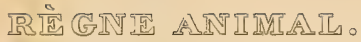

Qrachuides. PL. 3.
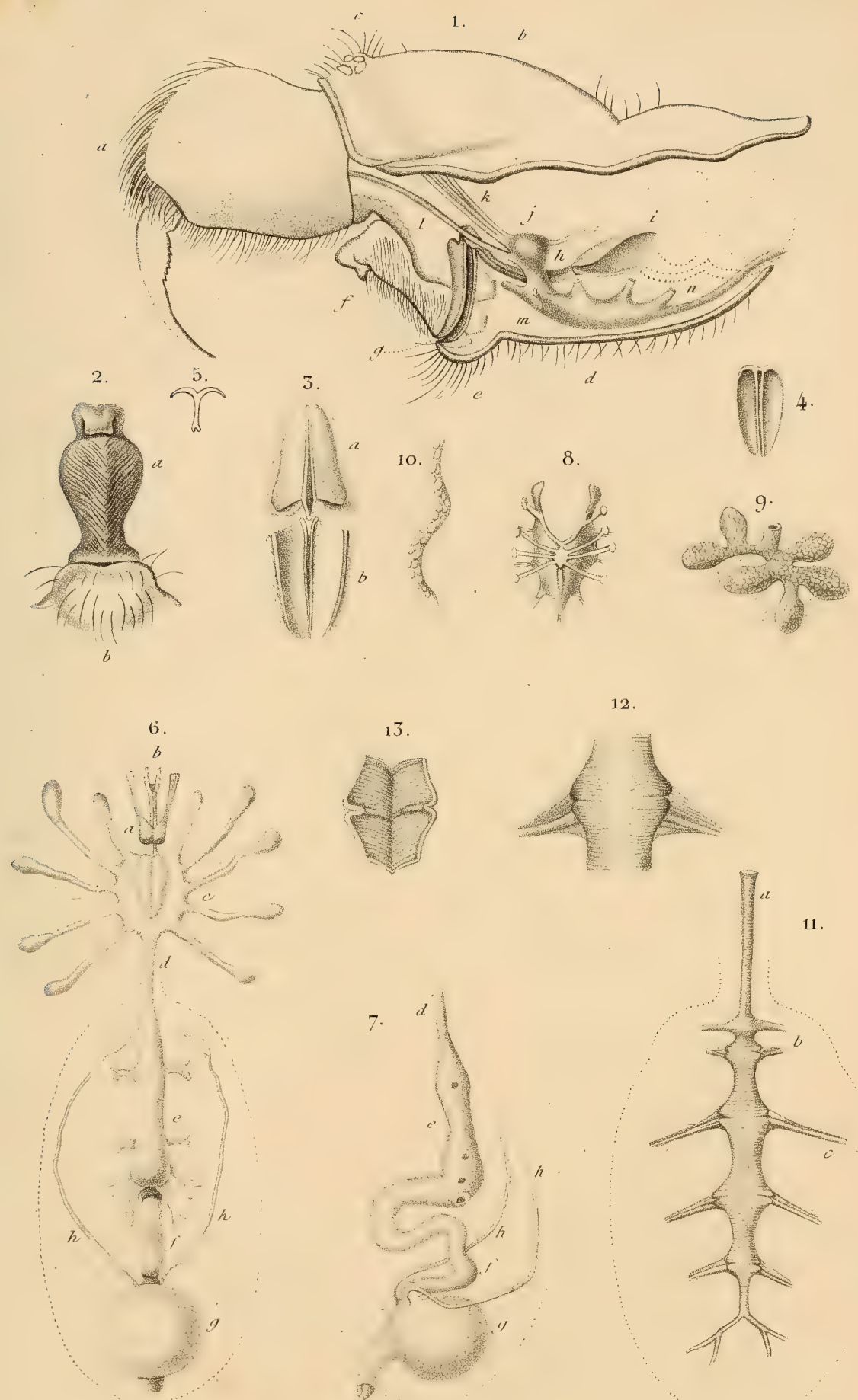

13.

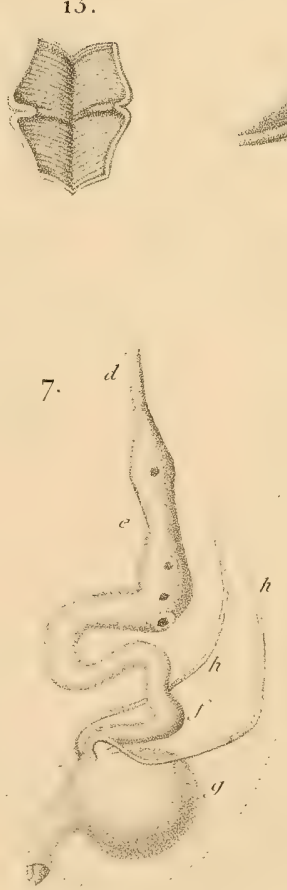

12.
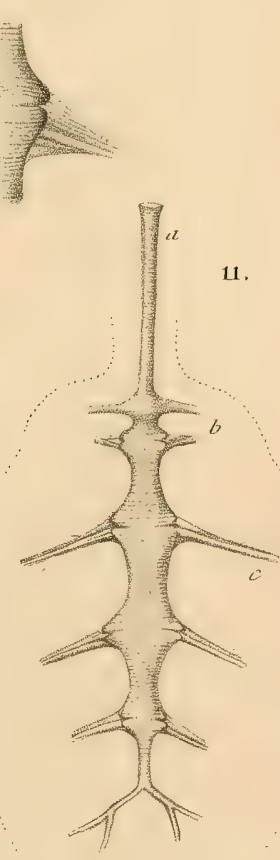

A. Dugies del

Murche Perrot se. 




\title{
ARACHNIDES PULMONAIRES.
}

\author{
ANATOMIE.
}

Fig. 1. Cour de la Clotho de Durand (Clotho Durandii. Lam.), arraché du corps et vu de profil, pour montrer l'insertion des vaisseaux. Grossi quatre fois.

Fig. 2. Cour de la Lycose narboninaise.

a. Muscles qui vont aux faux stigmates du dos. $-b$. Filamens vasculaires? qui les accompagnent; ce sont les vaisseaux pulmonaires de Treviranus.

Fig. 3. Coupe verticale de l'abdomen de la Mygale maçonne, montrant deux gros muscles fixés aux faux stigmates ou dépressions du dos.

Fig. 4. Segestrie perfide grossie quatre fois, vue en dessous, la peau et le plastron en partie enlevés pour montrer l'appareil trachéal qui coexiste avec les poumons.

$a$. Mandibule gauche. $-b$. Maxille droite. $-b$. Portion du palpe soutenue par la maxille gauche. - $c$. Lèvre. - $d$. $d$. Hanches. - $e$. Filières, dont quatre plus petites sont cacliées entre les quatre grandes; derrière est l'anus; devant, une petite tache cornée, triangulaire, servant à l'attacle d'un mnscle. - $f$. Poumon droit mis à découvert; son stigmate a été conservé arec la peau qui l'enviroune. $-g$. Deuxième stigmate communiquant avec l'appareil trachéal. - $h$. Cet appareil cumposé d'uu gros tube cartilagineux, d'où partent des écheveaux nombreux de trachées filamenteuses, parallèles, sous-divisées en faisceaux, et non ramifiées comrne celles des insectes. - $i$. Large faisceau de trachées mis à déconvert par l'ablation du plastron, formé par la réunion des deux écheveaux principaux dans lè pédicule abdominal; et dounant des fascicules à tous les membres.

Fig. 5. Une portion du poumon feuilleté de la Ségestrie vue au microscope.

Fig. 6. Idem de la Mygale maçonne.

Fig. 7. Idem du Pholeus phalangista.

Fig. 8. Un feuillet entier du poumon de la Mygale, vu à un for̀ grossissement.

$a$. Point d'insertion du support corné. - $b$. Extrémité libre.

Fig. 9. Les deux filières du côté droit de la Mygale maçonne.

$a$. Grande vésicule de l'organe sécréteur de la suie; il y en a cinq; le canal excrétcur de clacune va se terminer aux grosses canules centrales du dernier article de la filière. - $b$. Petites vésicules, entourées comme les grandes d'un tissu glaireux et terminées aussi chacune par un canal qui va anx petites canules du dernier article. $-c$. La petite filière portée sur une portion de peau à la base de la grande, $-d$. Premier article de celle-ci dépouillé de ses poils, pour laisser mieux voir son incisure spirale incomplète. - $e$. Deuxième article également dépouillé. - f. Troisième article auquel on a enlevé les poils et la majeure partie des petites cannles dont on ne voit que l'insertion.

Fig. 10. La pelite filière plus grossie, velue, mais sans orifice ui canule.

Fig. 11. Un poil.

a. Le poil. - Une petite canule $b$. et une grosse $c$. vues au microscope; c'est par ces dernières que sort la soie pour se furmer en filamens.

Fig. 12. Moitié inférieure de la coupe abdominale du Pholcus gihalangista mâle.

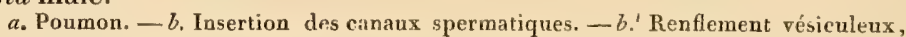
sorte de testicule. - $c$. Detix vésicules servant à la secrétion de la soie; c'est un excmple de la plus grande simplicité de ces organes. - $d$. Les deux caniıx excréteurs euveloppẻs d'une même membrane; jls vont aux petites filières $-e . f$. Vésicules des quatre autres filières. - g. Gros intestin rrjeté en arrière avec une portion de l'intestingrèle et des deux cauaux urinaires.

Fig. 13. Une des filières de la même Aranéide, avec le bout de son canal excréteur. 
RI足G

4.

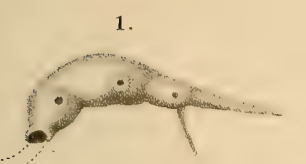

2.

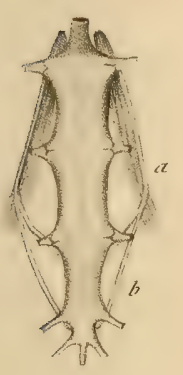

3.

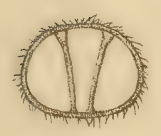

9.

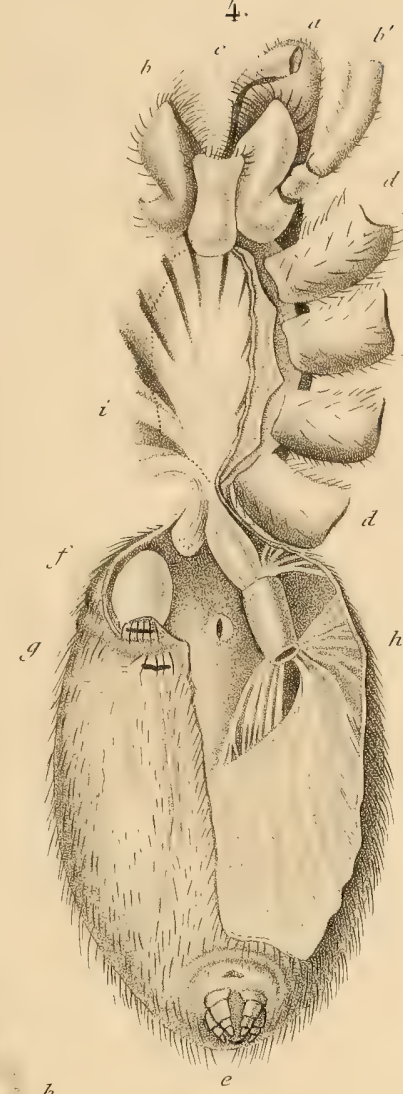

13.

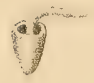

10.

(1)

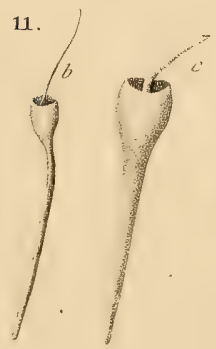

A. Dugès del.

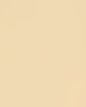

Crachuides. PL, 4.

8.

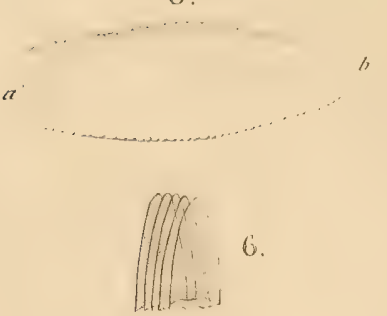

7.

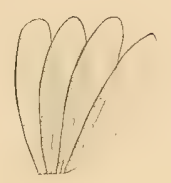

5.

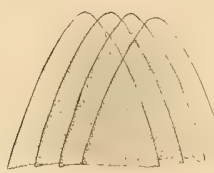

12.

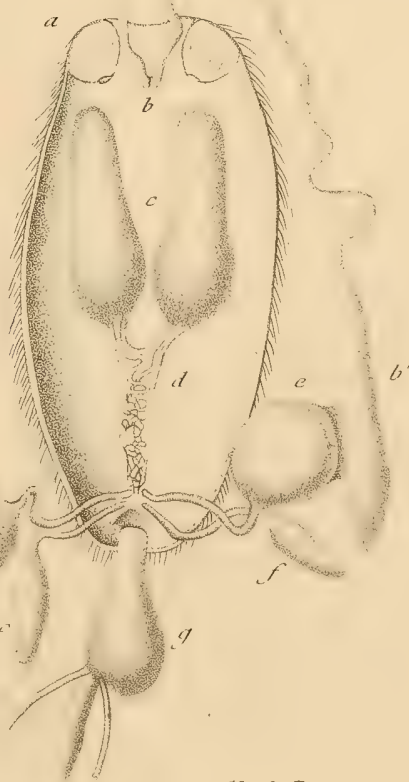

Marche Perrot sc, 




\section{ARACHNIDES PULMONAIRES.}

ARANÉIDES.

\section{S.Genre MYGALE. Mygale. Walck.}

Voyez, outre les figures ci-après, celles de la planche 1 .

Fig. 1. Une mandibule de la Mygale aviculaire grossie au double, vue par la face interne.

$a$. Crochet sans dentelures. - b. Poils simples de l'extrémité antérieure. - $c$. Poils plus longs couvrant la rainure destinée à recevoir le crochet; une partie a été rasée pour laisser voir les dents.

Fig. 1 a. Les yeux et le bandeau, trois fois aussi grands que nature.

Fig. 1 b. Houppes épaisses, serrées, veloutées, des deux derniers articles du tarse, grandeur naturelle.

Fig. 1 c. Une des innombrables soies qui les composent, très grossie.

Fig. 1 d. Extrémité plus grossie de la même desséchée; à l'état frais, elles sont sans doute molles et pendantes.

Fig. $1 e$. Un poil proprement dit, très grossi.

Fig. $1 f$. Bout du tarse dépouillé. La griffe paraît simple; tous les deux sont plats et exactement parallèles. Point d'ergot.

S.-Genre A'TYPE Atypus. Lat.

Fig. 2. ATYPE DE SUlzer, Atypus Sulzeri Latr., femelle; grandeur naturelle.

Fig. $2 a$. Longueur des pattes à partir de la ligne médiane du corps.

Fig. 2 b. Dessous du corps, grossi au double.

a. Mandibules $-b$. Maxilles. - $c$. Palpes. $-d$. Hanches entourant le plastron, dont une avance sert de lèvre, sans être mêcue séparée du reste par une dépression, comme chez la Mygale maçonne; on y voit des dépressions répondantaux ligamens de l'entothorax. - e. Plaque pulmonaire antérieure bornée par les stigmates et la rulve. $-f$. poumońs postérieurs, - $-g$. Les six filières. Latreille n'avait pas sans doute aperçu les deux plus petites.

Fig. $2 c$. Tubercule portant les yeux.

a. a. Bord inférieur du bandeau.

Fig. $2 d$. Une mandibule vuedu côté interne.

Fig. $2 e$. Une palpe du Mâle, d'après Hahn.

Fig. $2 f$. Les deux derniers articles d'une patte.

$a$. et $b$. Les neuf épines qui garnissent les quatre tarses antérieurs, $-c$. Les grandes griffes, dont une se voit montée. $-d$. L'ergot.

S..-Genre ERIODON. Lat.

Fig. 3 et $3 a$. Bouche et front de l'Eriodon occatorius. Lat. D'après Walckenaër.

S.-GENRe DYSDÈRE. Dysdera. Lat.

Ce sous-genre devrait être rangé parmi ceux du genre Araignée , près des Segestries.

Fig. 4. DYsdère ERYThrine Disdera erythrina Latr. femelle, grandeur naturelle. (Les couleurs sont variables du rouge brun au brun noir pour le corselet, et du jaune au violacé pour l'abdomen.)

Fig. $4 a$. Longueur respective des pattes.

Fig. $4 \mathrm{~b}$. Corps vu en dessous, trois fois aussi grand que nature.

$a$. Mandibules. $-b$. Un palpe. - $c$. Maxilles, $-d$. Hanches. $-e$. Ylastron entouré d'une bande cornée noirâtre dans laquelle sont percés les trous pour l'insertion des pattes, marquée ici en blanc par les chairs qui les remplissent. $-f$. Pièce cornée du pédicule, large et embrassante. - - g. Plaques pulmonaires, stigmates antérieurs, et entre eux, la vulve. - $h$. Stigmates postérieurs conduisant à des trachées comme chez les Ségestries (Voyez pl. IV, fig. 4). - $i$. Filière rentrée.

Fig. $4 c$. Les yeux.

Fig. $4 d$. Filiềres poussées en dehors et plus grossies que dans la fig. $4 b$.

Fig. $4 e$. Palpe du mâle, côté externe.

Fig. $4 f$. Les deux derniers articles du tarse de la troisième patte.

a. Avant-dernier article armé d'épines. $-b$. Dernier article fort court, terminẻ par deux griffes dentelées $c$. et une double houppe $(d$.) propre à adkérer aux corps polis.

Fig. 4 g. Une des soies de cette houppe. 

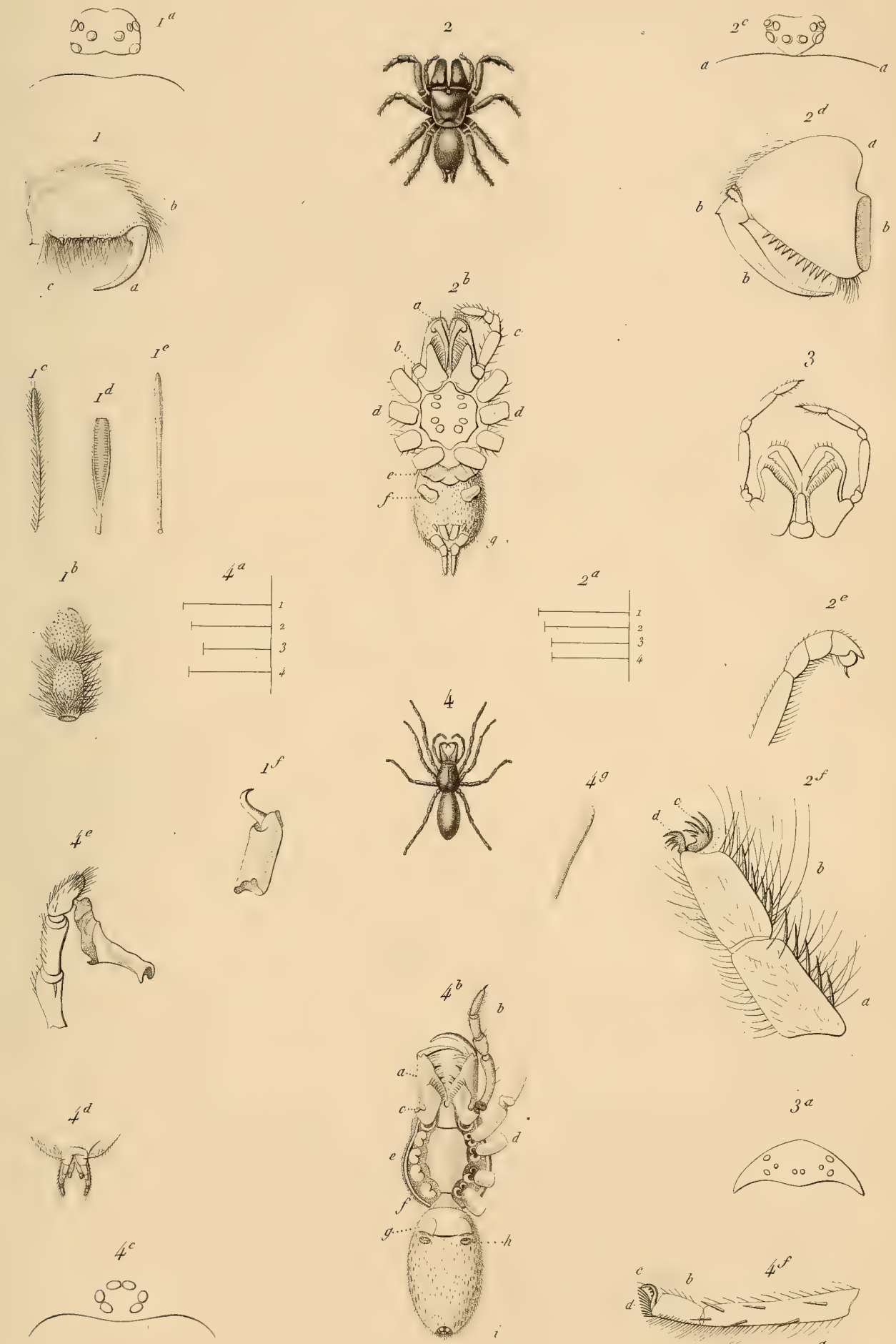

A. Dugies del

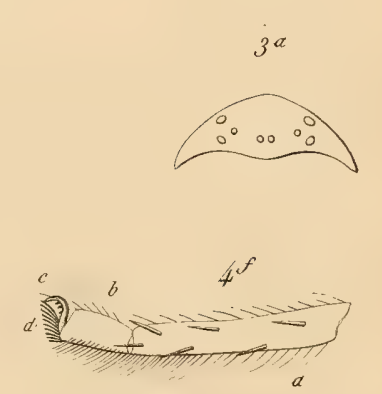

Schmelz so

2. ATYPE $7 E$ SULZER. . (Atypus Sulzeri. LaR:)

4. DYSDERE ERYTHRINE. (Dysdera crythrina. Latr.) 




\section{ARACHNIDES PULMONAIRES.}

\section{ARANEIDES.}

Genre MYGALE.

S.-Genre FILISTATE. Filistata. Lat.

(Ce sous-genre devrait rentrer aussi dans le genre suivant.)

Fig. 1. Filistate Bicolone. Filistata bicolor Latr. Femelle de grandeur naturelle.

Fig. 1 $a$. Longueur des pieds.

Fig. 1 b. Les yeux et le front; grossissement considérable.

Fig. 1 c. La bouche, etc.; grossissement quadruple.

La bouche suivie du plastron sternal $c$, entourée parles hanches $d$, et donnant une avance ponr représenter la lèvre. - $a$. Mandibules. - b. Maxilles.

Fig. 1 d. Filière grossie environ huit fois, poussée en dehors par la pression de l'abdomen.

a. Double plaque servant à une insertion de muscles; derrière sont les deux filières moyennes, soudées, mais distinctes. $-b$. Valvule qui courre l'anus. $-c \cdot d$. Filières antéricures et postérieures.

Fig. 1 e. Abdomen vu de côté, grossi trois fois.

$a$. Pédicule. $-b$. Piaque vulvaire ou épigyne, et ensuite plaque pulmonaire avec son stigmale, derrière lequel est un faux stigmate servant intérieurement à une insertion musculaire, et qui a pu faire croire faussement à l'existeuce de quatre poumons chez cette aranéide: - $c$. Les filières et Ja valvule anale.

Fig. 1 f. Un poil plat et frangé; ils sont appliqués à plat sur la peau, surtout aux paltes.

Fig. 1 g. Corselet du mâle quadruplé; il est un peu moins étroit que celui de la femelle.

$a$. Mandibules peu saillantes. $-b$. Hanche de la première patte. $-c$. Palpe.

Fig. 1 h. Extrémité plus grossie de ce palpe.

Fig. 1 . Une griffe el l'ergot, ordinairement cachés dans les poils.

S.-Genfe CLOTHO. Clutho Walck.

Fig. 2. Clotho de durand, Clotho Durandii Latr. Femelle, de grandeur naturelle.

Fig. 2 a. Longueur des pattes.

Fig. 27 . Les yeux et le front.

Fig. 2 c. La bouche.

$a$. Mandibules un peu pơussées en avant. $-b$. Maxiß̊les. $-c$. Premiers articles du palpe. - $d$. Lèvré peu ou point molile, quoique séparée du plastron $e$. par un sillon profond. $-f$. Une hanche.

Fig. $2 d$. Extrémité d'une des pattes antérieures, avec ses épines, une des griffes et l'ergot.

Fig. 2 e. Palpe du mâle vu en dessous.

$a, b$. Les deux premiers articles. - $c$. Le dernier, - $d$. Organes copulateurs en partie recouverts par celui-ci.

Fig. 2 f. Abdomen vu de profil; double grandeur.

a. Gibbosité. - b. Pédicule - $-c$. Uue des plaques pulmonaires arec son stigmate, derrière lequel est un faux stigmate. $-d$. Epigyne. $-e$. Une des filières moyennes précédée et suivie de l'antérieure et de la postérieure. $-f$. Tubercule anal.

Fig. $2 g$. Extrémité postérieure du corps, figurée en dessous à un très fort grossissement.

$a$. $a$. Filières antérieures; entre elles sont les moyennes, $-b$. $b$. Filières postérieures et entre elles le tubercule anal ayant l'anus à sit base, unc aigrette à son sommet, et une double curonne de soies rigides et lougues.

Fig. $2 h$. Grossissement considérable d'un bout de ces grandes soies.

Fig. $2 i$. Habitation d'une jeune Clotho; celles des aduites sont rarement aussi régulières. 

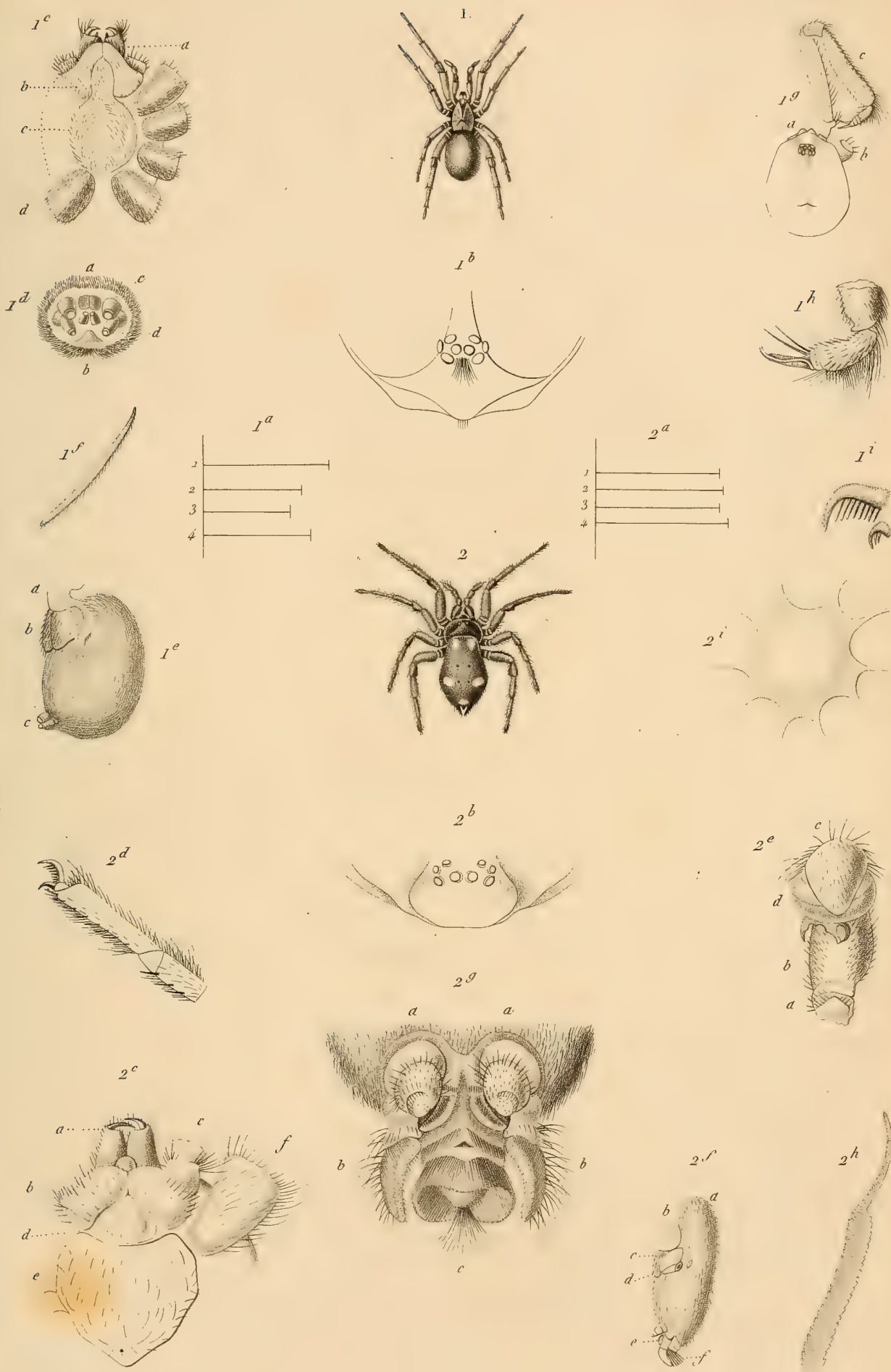

A. Dugies del

1. FILISTATE BICOLORE. (Filistata bicolor. Latr.)

2. CLOTHO DE DURAND. (Clotho Jurandi. LaT.) 




\title{
ARANÉIDES.
}

\section{GENRE ARAIGNÉE.}

\author{
S.-Genre DRASSE. Drassus. Walck.
}

Fig. 1. Drasse méla Ogaster. Drassus melanogaster. Lat. D. Lucifugus. Walck. Mâle entre l'état de première enfance où il ressemble tout-àfait à la femelle, et l'état adulte où il a les pattes plus longues, le corselet plus fort et le ventre beaucoup moindre.

Fig. 1 a. Longueur des pattes.

Fig. 1 b. Le même vu en dessous ; grossissement au triple.

a. Un palpe dont le bout est simplement renflé, après un troisième changement de peau il offrirait en dessous une excavation contenant des pièces copulatrices fort peu saillantes.- $b$. Mandibules, suivies des maxilles et de la lèvre.- $c$. $c$. Les hanches, et entre elles, le plastron. - $-d$. Opercules pulmonaires et entre eux deux faux stigmates. - $e$. Pli transversal séparant la partie thoracique de l'abdominale dans le ventre ou thoracogastre; on y voit l'ouverture des organes génitaux. Derrière ce pli sont deux rangées longitudinales de cicatricules $(f)$ qui se retrouvent dans beaucoup d'autres aranéides et correspondent au trajet des grands muscles droits. - g. Les six filières dont les antérieures sont les plus grosses et les plus longues; les derniers articles rentrent dans le premier.

Fig. $1 \mathrm{c}$. Le front et les yeux du même. Bandeau assez large, yeux latéraux très écartés.

Fig. 1 d. Une portion du palpe de la femelle;

Fig. 1 e. Deux articles du tarse d'une des quatre pattes antérieures.

$a$. Une des griffes pectinées; il n'y a pas d'ergot.-b. b. Houppes de soies spatulées; il n'y en a pas aux quatre pattes postérieures. $-c$. $c$. Piquans.

Fig. $1 \mathrm{f}$. Une des soies spatulées et granuleuses des houppes, très grossie.

Fig. 2. Les yeux du D. fuscus. Lat. D. Montanus. Hahn; le D. segestri. formis, L. Dufour, a les yeux disposés de même; il n'est donc pas identique avec le précédent, comme l'a pensé Latreille. Bandeau presque nul; yeux latéraux, plus rapprochés que les médians.

\section{S.-Genre SEGESTRIE. Segestria. Lat.}

Fig. 3. SÉgestrie PERFIDE. Segestria perfida, femelle, de grandeur naturelle, mandibules vues obliquement ; un peu en raccourci.

Fig. 3 a. Longueur des pattes.

Fig. 3 b. Les yeux.

Fig. 3c. Bout du palpe malle, vu par son côté interne.

Fig. $3 d$. Bout d'une patte avec une grande griffe et l'ergot.

Fig. 4. Le ventre du mâle vu en dessus. Il présente la livrée de la femelle dans le jeune age; elle a alors les mandibules brunátres, et on en a fait à tort une espèce sous le nom de $\boldsymbol{S}$. Senoculata. (Walck).

(D'après nature.) 

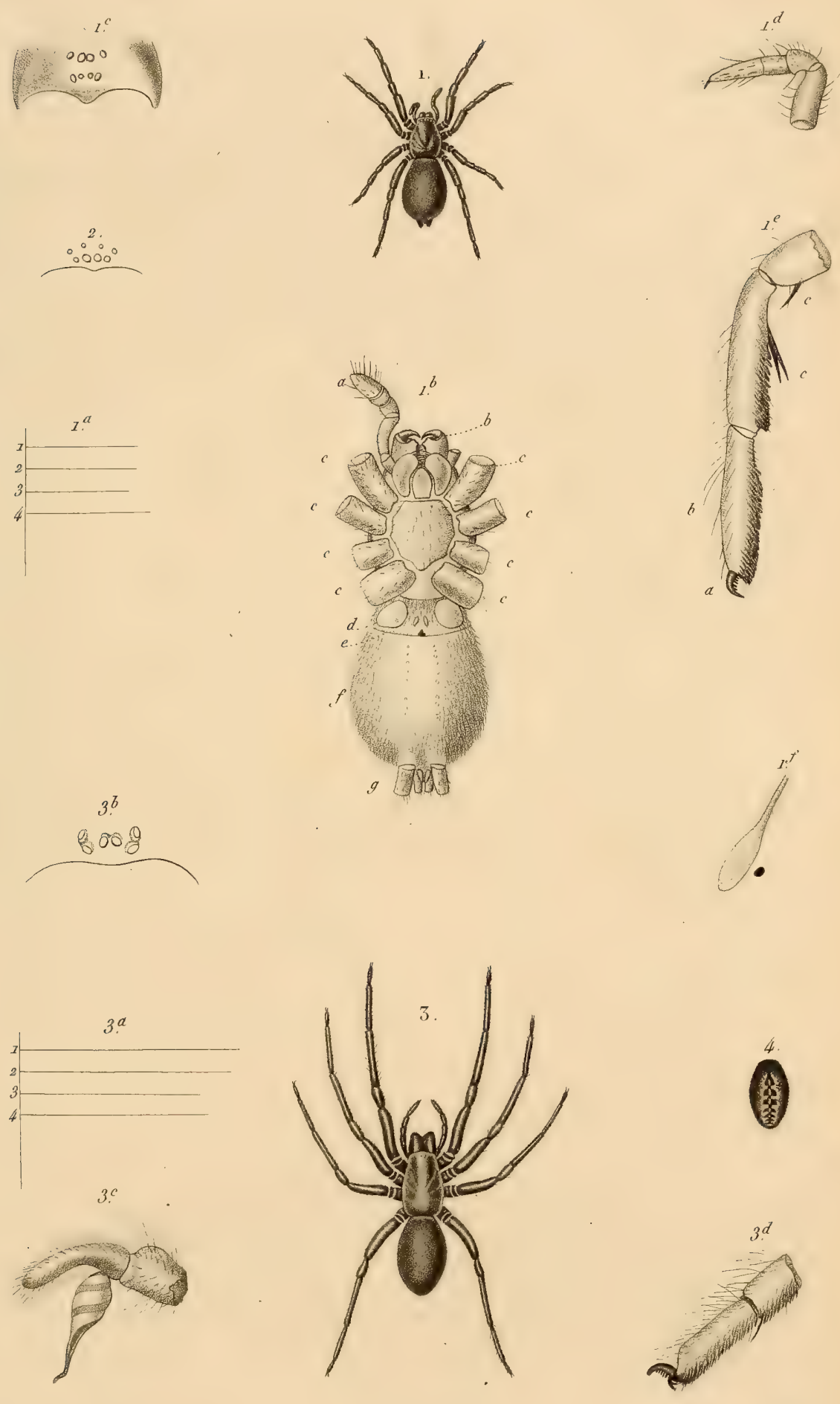

iès.

Oudet sc. 




\section{ARANÉIDES.}

Genire ARAiGnÉE.

S.-Genke CLUBIONE. Clubiona. Lat.

Fig. 1. Clubione nourrice. Clubiona nutrix. Mâle adulte, de grandeur naturelle, les mandibules étendues en avant.

Fig. 1 a. Tronc de la Clubione nourrice femelle vue en dessous; grandeur naturelle. Les mandibules sont aussi projetées en avant, ce qui est une position forcée; les filières postérieures se montrent déjà plus longues que les antérieures; leur dernier article assez court se fléchit en dedans un peu comme chez les Aranea.

Fig. $1 b$. La tête $(a)$ de la même vue en dessus. Grossie au double; b. Mandibules dans leur position la plus ordinaire, vues en raccourci.

Fig. 1 c. Longueur des pattes chez la C. nourrice femelle.

Fig. $1 d$. Le front et les yeux; triple grandeur. Leur type est celui de plusieurs Clubiones, l'atroce, lerratique, la trompeuse; il se rapproche des Théridions et des Epéires.

Fig. 1 e. Extrémité du palpe vu par le côté externe, emprunté au mâle de la $C$. erratica; il diffère à peine de celui du précédent.

Fig. $1 f$. Extrémité du tarse. Les griffes cachées par les houppes de soies prenantes qui occupent aussi l'avant-dernier article; il en a à toutes les pattes.

Fig. $1 \mathrm{~g}$. Lìvre et maxilles de la même très grossies.

Fig. 2. Le front et les yeux de la C. soyeuse; grossissement en quintuple. Ce type est celui de plusieurs autres espèces très allongées, $C$. lapidicole, etc.

Fig. $2 a$. Longueur des pattes chez la C. soyeuse femelle.

S.-Genre ARAIGNÉ proprement dite. Aranea. Lat.

Fig. 3. AraignéE domestioue. Aranea domestica. Femelle; grandeur naturelle, pattes forcément étendues.

Fig. $3 a$. Longueur des pattes.

Fig. 3 b. La bouche plus que quadruplée. $a$. Plastron. $b, b$. Premières hanches. $c, c$. Premier article des palpes porté par les maxilles, entre lesquelles est la lèvre. $d, d$. Mandibules tirées en avant.

Fig. $3 \mathrm{c}$. La tête vue de profil à un grossissement de trois fois environ.

$b$. Plastron.-g. Les insertions des deux premières pattes. - $d$. Palpe.-a. Mandibule surmontée d'un condyle articulaire, et terminée par le crochet vu en raccourci. $c$. Maxille. - e. Lèvre.-b. Carapace portant les yeux. Cette figure et la précédente sont destinées à offrir le type de bouche caractéristique du grand genre Araignée. Comparez la précédente avec la fig. 8. pl. 2 , et celle-ci avec les fig. I $b$. HI. I; fig. 4 . pl. 2; fig. I. pl. 3; fig. I et $2 d$. pl. 5 .

Fig. $3 d$. Bout du palpe chez le mâle pubère, vu du côté interne.

Fig. $3 d$ '. Le même avant la puberté.

Fig. $3 e$. Le front et les yeux; grossis trois fois et demie environ.

Fig. 3f. Bout d'une patte.

Fig. $\mathbf{3} g$. Les six filières et l'anus.

Fig. 3h. Une des canules à soie $(a)$ et un des poils simples (b) qui y sont mèlés, et les cachent sous les longues filières qu'on a appelées palpes de l'anus, dans la fausse persuasion qu'elles ne sont point perforées.

Fig. 3i. Un poil plumeux. On en trouve partout, même sur les pattes, mèlés à des poils simples comme celui de la figure précédente.

(D’aprés nature.) 

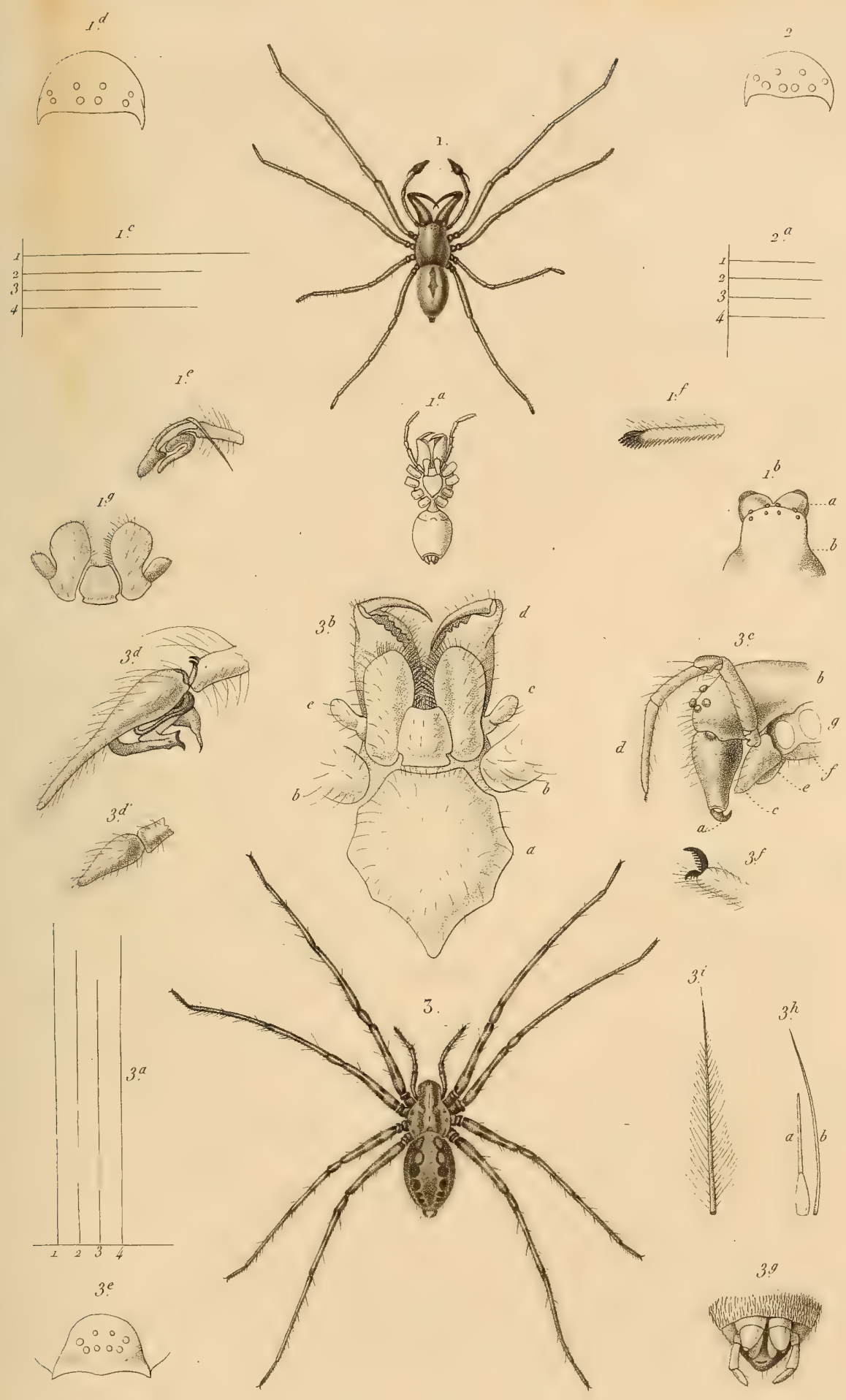




\title{
ARACHNIDES PULMONAIRES.
}

\author{
ARANËIDES.
}

Genre ARAIGNÉE.

Suite du S.-Genre ARA IGNÉE proprement dite. Aranea. Latr.

Fig. 1. Le front et les yeux de l'Araignée labyrinthe mâle; dans la femelle les yeux du milieu sont un peu moindres, quoique toujours plus grands que les autres. Grossissement quintuple.

Fig. 1 a. Lèvre et mâchoire grossie trois fois et demie seulement.

Fig. 2. Front du nysse pédicolore, d'après Walkenaër. Cette espèce paraît réellement devoir rentrer dans le sous-genre Aranea.

Fig. $2 u$. La bouche du mème sans les mandibules, d'après le même naturaliste.

S.-Genre ARGYRONÉTE. Argyroneta. Latr.

Fig. 3. ARgyronète AQUatique. Argyroneta aquatica. Latr.; figure réduite à la grandeur naturelle, d'après celle très grossie de Hahn.

Fig. $3 a$. La bouche, d'après Walkenaër.

Fig. 3 b. Le front et les yeux, aussi d'après ce zoologiste.

S.-Genre SCYTODE. Scytodes. Latr.

Fig. 4. Scytode thonaciQue. Scytodes thoracica. Latr. Femelle; doublée.

Fig. $4 a$. Longueur respective des pattes, aussi au double.

Fig. 4 b. La bouche et le plastron; grossis sept à huit fois. Les mandibules un peu portées en avant.

Fig. 4 c. Le bout d'une des mandibules, écrasé et vu au microscope.

Fig. $4 d$. Le devant du corps, offrant le front les yeux, les mandibules; grossissement de sept à huit diamètres.

Fig. 4 e. Extrémité d'une patte, vue au microscope, pour montrer les griffes portées sur un article supplémentaire.

Fig. 4 f. Une des soies dentelées qui partent de l'article précédent, bien plus grossie encore.

Fig. 5. Longueur respective des pattes de la Scythode blonde (Duf.) femelle. Chez le male la première et la quatrième patte sont égales.

Fig. 5 a. Le céphalothorax, vu en dessus, grossi quatre fois et demie environ.

Fig. 5 b. Le même, vu par-devant, grossi sept fois.

Fig. 5 c. Le bout du palpe mâle, vu en dehors, considérablement amplifiée.

S.-Genre PHOLQUE. Pholcus. Walck.

Fig. 6. Pholque phalangioïde. Pholeus phalangioĩdes. Walck. Femelle, grandeur naturelle, pattes étalées.

Fig. 6 a. Mesure des pattes.

Fig. 6 b. La bouche et le plastron.

Fig. 6 c. Le céphalothorax, vu par-devant, montrant de face le grand bandeau qui sépare les yeux de l'insertion des mandibules.

Fig. $6 d$. Extrémité d'une patte, vue au microscope.

Fig. 6 e. Un palpe mâle très grossi, vu par son côté interne.

a. Maxille, A' son point d'insertion. - $b$. Trokanter, D, E, article copulateur très complexe, mu par des muscles puissans contenus dans l'article $C_{\text {. On y remarque }}$ surtout une vésicule $D$ et un conduit excréteur perforé $F$.

Fig. 7. Front du Pholcus sexoculatus. Nob. 


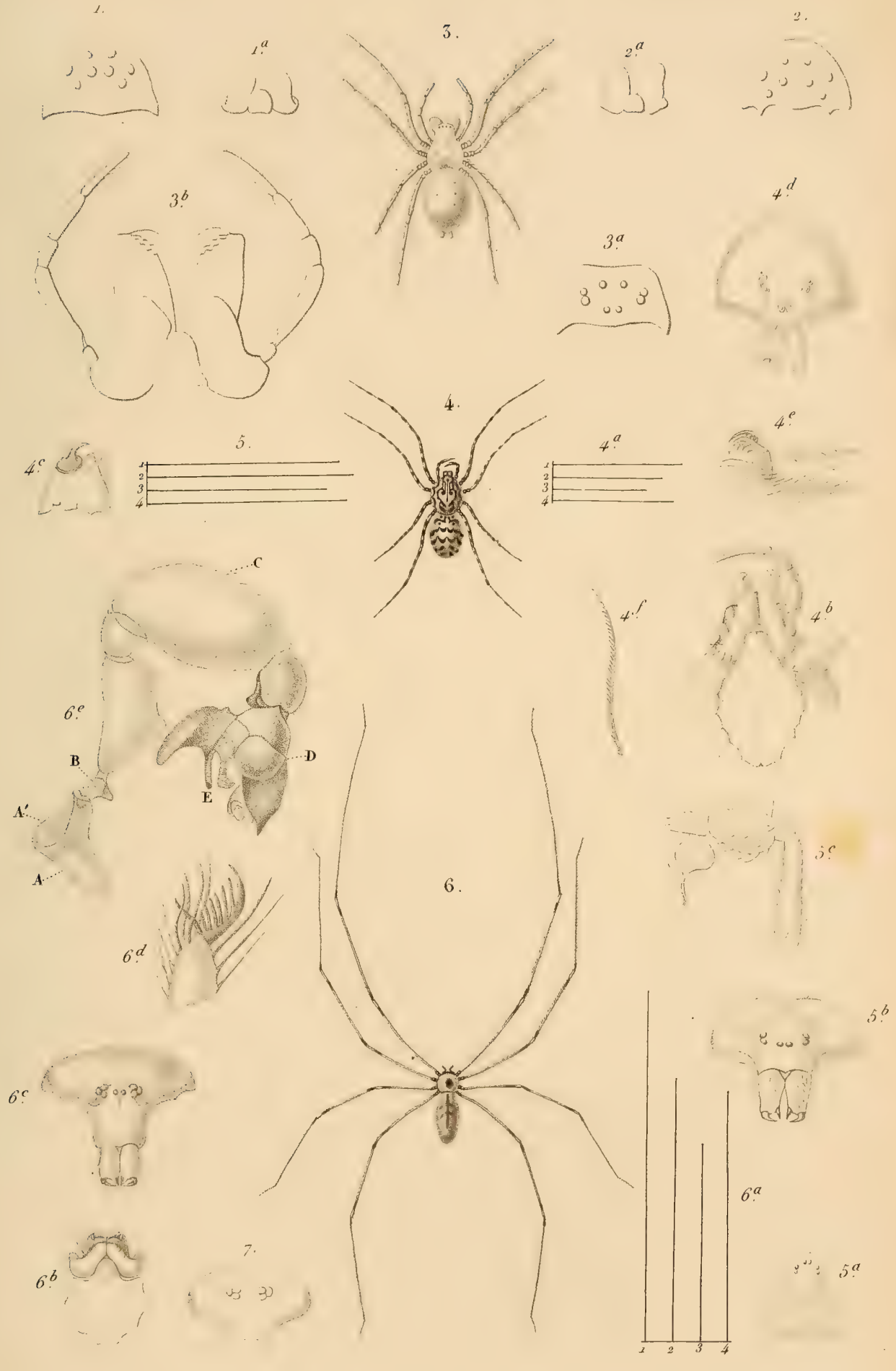

- A.Dugès del.

I.anvin sc 




\title{
ARACHNIDES PULMONAIRES.
}

\author{
ARANËIDES.
}

\section{Genre ARAIGNÉE. Aranea.}

\section{S.-GENRE THÉRIDION. Theridion. Walck.}

Fig. 1. Théridion bienfaisant. Femelle grossie trois fois en diamètre.

Fig. 1 a. Longueur des pattes triplées aussi $(1,2,4,3$.)

Fig. 1 b. Plastron, lèvre et maxille très amplifiés.

Fig. 1 c. Le front et les yeux.

Fig. 1 d. Un des poils, vu au microscope.

Fig. 1 e. Une des griffes et l'ergot.

Fig. 1 f. Palpe du mâle, vu du côté externe. Grossissement considérable.

Fig. 2. Longueur des pattes du THéridon a TREIze TAGHES (Theridion tredecim-guttatum), ou Malmignatte; type du genre Latrodecte de Walck. (1, 4, 2, 3.) Grandeur naturelle.

Fig. $2 a$. Céphalothorax du même, vu par-devant, grossi cinq fois environ.

Fig. $2 b$. Extrémité d'une patte, grossie trois fois. On y remarque la gracilité comparative des deux derniers articles, et la grandeur de l'ergot égale à celle des griffes.

Fig. 2 c. Les filières et la valvule anale disposées en rosette.

S.-GENRE LINYPHIE. Linyphia. Latr.

Fig. 3. LINYPHIE MONTAGNARde.Linyphia montana. De grandeur naturelle.

Fig. 3 a. Corselet de la femelle, vi en dessus; grossi quatre fois et demie.

Fig. 3 b. Longueur des pattes de la femelle.

Fiy. $3 c$. Jue front et les yeux de la même.

Fig. $3 \mathrm{~d}$. Les lèvres et les maxilles, grossies environ quatre fois.

S.-GenRe ULOBORE. Uloborus. Latr.

Fig. 4. Ulobore de Walckenaer. Uloborus Waickenaërius, femelle; de grande taille.

Fiy. $4 a$. Dessus du corps amplifié pour en montrer les lanuginosités.

Fig. $4 \mathrm{~b}$. Un des poils plumeux qui les forment; les barbes étalées sous. le microscope.

Fig. $4 c$. Longueur des pattes, doublée d'après un individu de taille médiocre.

Fig. $4 d$. Lèvres et maxilles.

Fig. $4 \mathrm{e}$. Corselet, vu par-devant.

Fig. 4 f. Cocon grossi au double.

S.-Genre TÉTRAGNATHE. Tetragnatha. Latr.

Fig. 5. TÉtragnathe Étendue. Tetragnatha extensa, femelle, grandeur naturelle.

Fig. 5 a. Longueur des pattes.

Fig. 5 b. Bouche et plastron du male.

$a$. Plastron. $-b$. Lèvre surmontée d'un labre. $-c$. Maxilles. $-d$. Une mandibule un peu ples grande que celle de la femelle, et armée de dents plus longues. - 6 . Un des palpes.

Fig. 5 c: Le devant de la tête. 


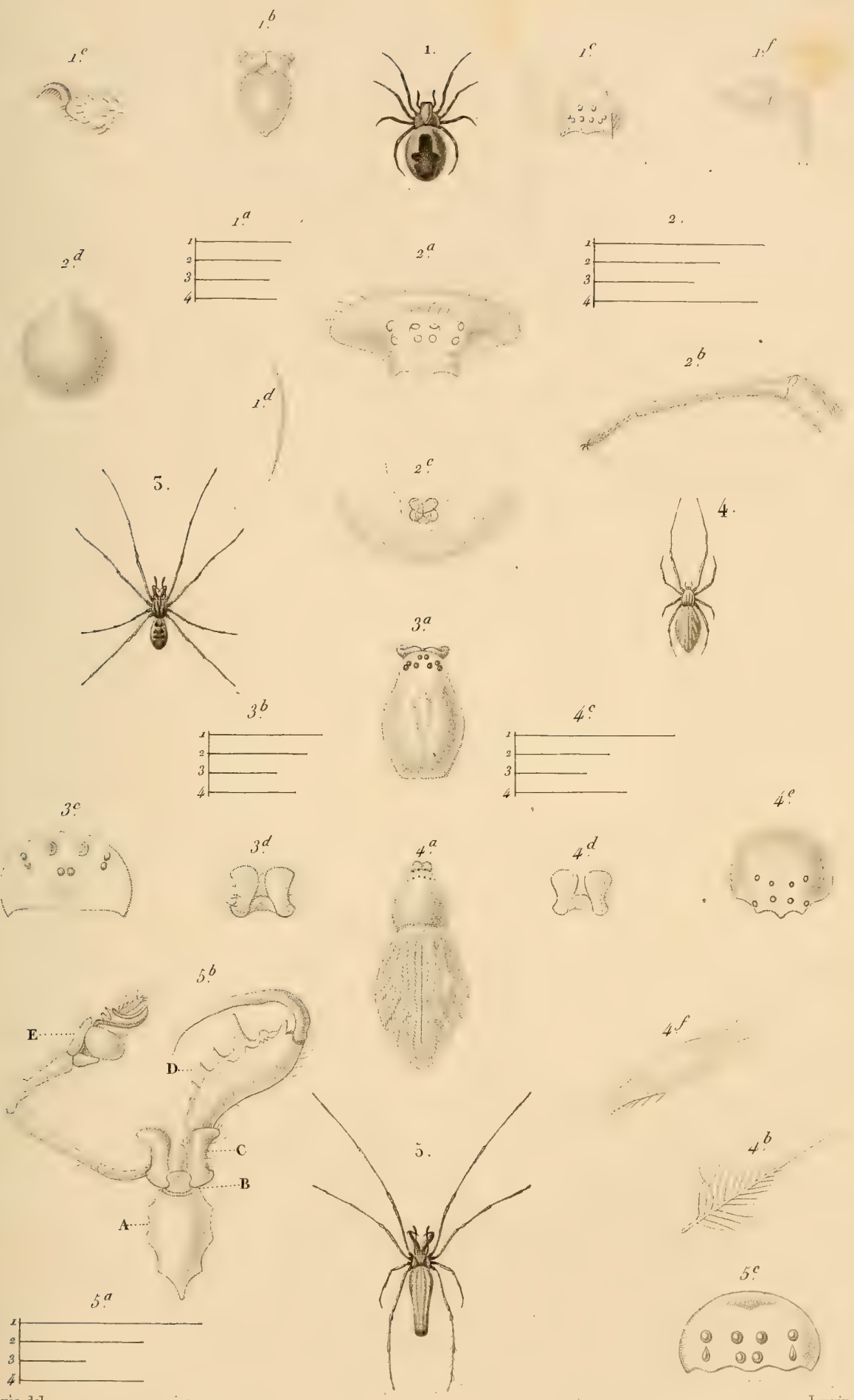

A. Dugès del.

Lanvin ac

1. THÉRIDION BIENFAISANT" (Theridion benignum, ) 4.ULOBORE DE WALCKENAËR, (Ulobores Walckenaërius)
3.LINYPHIE MONTAGNARDE : (Linyphia montana.) 5.TE'TRAGNATHE ÉTENDUE, (Tetragnata extensa) 




\title{
ARACHNIDES PULMONAIRES.
}

\author{
ARANEIDES.
}

Genre ARAignéE. Aranea. Latr.

S.-GenRe EPÉIRE. Epeira. Walck.

Fig. 1. EPÉIRE FAsciéE. Epeira fasciata. Femelle, grandeur naturelle.

Fig. 1 a. Longueur des pattes.

Fig. 1 b. Epéire fasciée, vue en dessous, grossie au double pour faire voir successivement de haut en bas.

$a$. Les mandibules. $-b$. Les maxilles portant les palpes à leur base, et serrant la lèvre entre elles. $-c$. Le plastron entouré des hanches. - $d$. La plaque triangulaire du pédicule abdominal suivie de deux faux stigmates cornés, servant intérieurement à l'attache des muscles. - e. Les deux opercules pulmonaires striés du côté interne où ils couvrent les feuillets du poumon. - $f$. Les deux grands stigmates et entre eux l'épigyne. - $g$. Tout-à -fait en arrière les filières et la valvule anale.

Fig. $1 c$. Le front et les yeux.

Fig. 1 d. Une des grandes griffes et l'ergot.

Fig. 1 e. Le mâle de l'Épéire fasciée adulte, grandeur naturelle.

Fig. $1 \mathrm{f}$. Son palpe très grossi, vu en dehors.

Fig. 1 g. Cocon de cette Épéire.

Fig. $1 \mathrm{~h}$. Coupe dı mème pour montrer le sac central suspendu au milieu d'une bourre fort lâche.

Fig. $2 a$. Le front et les yeux de l'ÉpÉIRE DIADÈMe.

Fig. 2 b. L'épigyne de la même fort différent de celui de la fasciée, vu de profil avec une portion de la peau de l'abdomen qui porte l'opercule pulmonaire e ton stigmate.

Fig. 3. ÉpÉIRe CURVIGAUde. Épeira curvicauda. Vautier. Grandeur naturelle. Ces Épéires épineuses constituent le sous-genre Acrosoma de Hahn, Microthena et Gasteracanthi de Sundeval, Plectana de Walckenaër.

\section{S.-Genre MiGROMMATE. Micrommatn. Latr. Sparassus. W.}

Fig. 4. Micrommate smaragdine. Mierommata smaragdula. Femelle, grandeur naturelle.

Fig. 4 a. Longueur des pattes.

Fig, 4 b. Lèvres et maxilles.

Fig. 4 c. Front et yeux.

Fig. $4 d$. Pa! pe mâle, vu en dehors.

Fig. 4 e. Tarse de la temelle; les griffes sont plus dégagées chez le mâle. on voit ici une houpe terminale et des brosses aux deux derniers articles. 

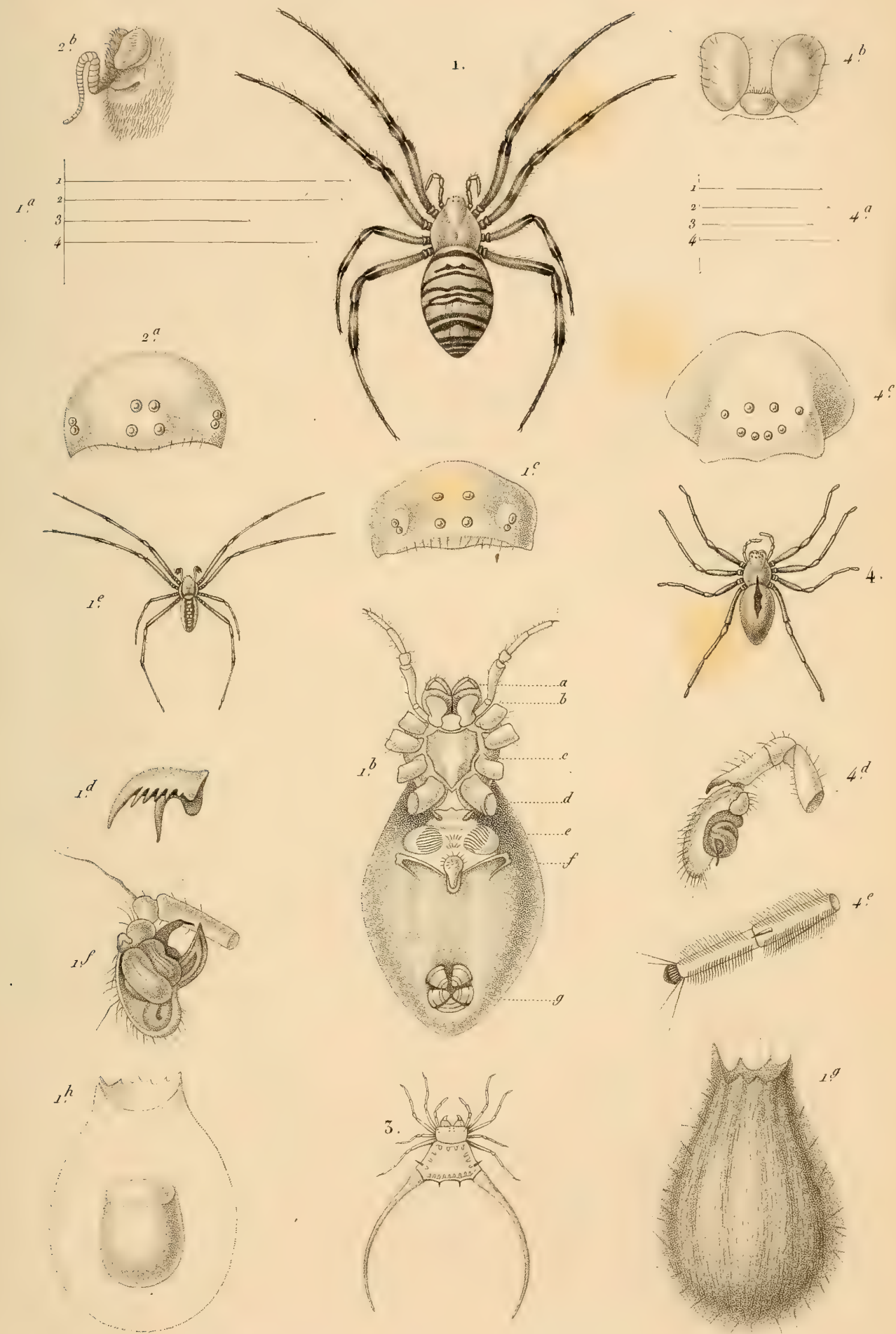

Dugies del
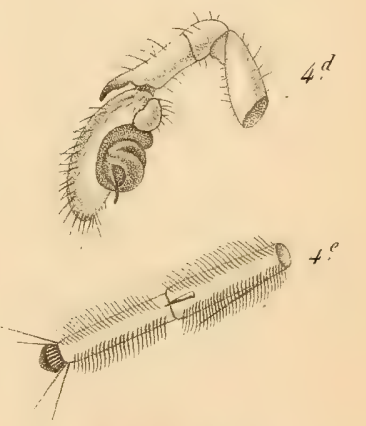

I. EPEÏRE FASCIÉE. (Épeira fasciata, Walck.)
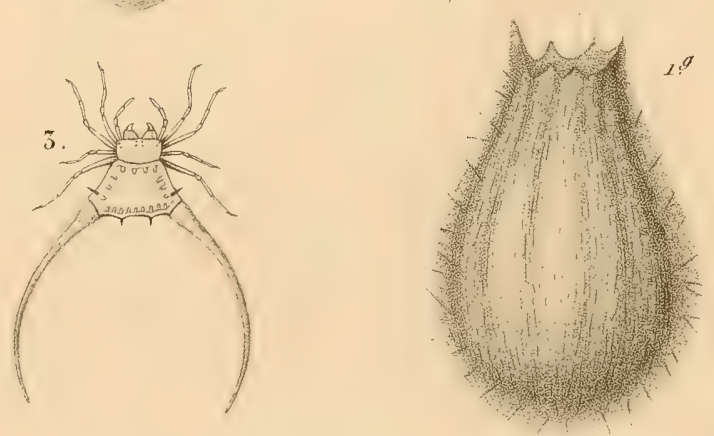

Giraud sc.

3.EPEIIRE CURVICAUDE. (Epcira curvicauda, Vauthier.) 




\title{
ARAGHNIDES PULMONAIRES.
}

\author{
ARANÉIDES.
}

Genre ARAignéE. Aranea. Latr.

S.-Genre SÉLÉNOPE. Selenope. Dufour.

Fíg. 1. SÉlÉnOPe Onalosone. ( $S$. omalosoma.) Femelle, grandeur naturelle. D'après M. Léon Dufour.

Fig. 2 a. Longueur proportionnelle des pattes de la Sélénope d'Égypte très augmentée.

Fig. 2 b. Le front, les yeux et la mandibule de la même.

Fig. 2 c. Lèrre et maxille de la mème.

Fig. $2 d$. Le bout d'une patte. (Ces quatre figures sont copiées de l'ouvrage d'Egypte.)

S.-Genre PHILODROME. Philodromus. Walck.

Fig. 3. Philodrome rhombifìre femelle, grandeur naturelle.

Fig. 3 a. Longueur des pattes au double.

Fig. 3 b. Corps du male très grossi.

Fig. 3 c. Le front et les yeux de la femelle.

Fig. 3 d. Lèvre et mâchoire du mâle.

Fig. 3 e. Un des poils plumeux et écailleux de l'abdomen.

(Les pieds sont à houppes et ả brosses comme ceux des Micrommates, caractère important souvent omis par Latreille et que nous avons donné a vec soin dans nos figures quand il existe.)

S.-GeNre THOMISE. Thomisus. Walck.

Fig. 4. Тномise Ciтron, Thomisus citreus, femelle, grandeur naturelle.

Fig. 4 a. Longueur des pattes au double.

Fig. $4 . b$. Lèvre, maxilles et un palpe femelle.

Fiy. $4 \mathrm{c}$. Front et yeux à éminence qui porte en arrière les yeux extérieurs et supérieurs.

Fig. $4 d$. Extrémité d'une des pattes antérieures. (Point de houppe ni d'ergot.)

Fig. 4 e. Palpe mâle vu en dehors, appartenant au T. bordé (T. liturutus, Walck), que je crois être le màie du T. crêté. ( $T$. cristatus, Walck. viaticus, L Cierkii, Sav. et Aud.)

S.-Genre STORENE. Storena. Walck.

Fig. $5 a$. Lèvre et máchoire de la sTORÈnE HLEUE.

Fig. 5 b. Les yeux. Ges deux figures sont coptées de Walck.

S.-Genre OXYOPES. Oxyopes. I.atr. Sphasus. Walck.

Fig. 6. OXYOPE PANACHÉ (O.variegatues, lineatus. Latr. Sphasus hetero phtialmus et italicus, Walck.) Femelle, grandeur naturelle.

Fig. 6 a. Longueur des pattes.

Fig. 6 b. Le front et les yeux.

Fig. 6 c. Lèvre et maxilles.

Fig. 6 d. Une des écailles du dos, dont la conservation plus ou moins complète fait varier les dessius.

Fig. 6 e. Un piquant des pattes.

S.-Genre CTENE. Ctenus. Walck.

Fig. 7 a. Lève el machoires du crère pourtux, diaprès Walck.

Fig. 7 b. Les yeux du mème. 
1.

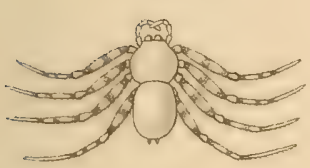

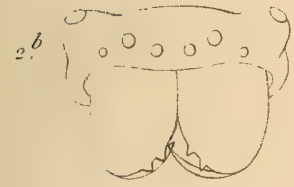
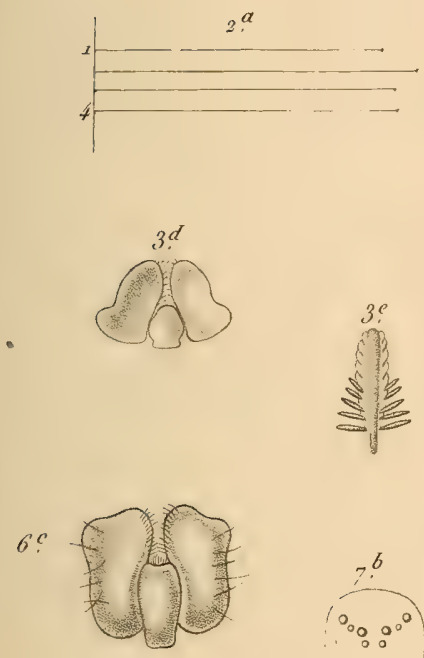

$6 ! \sqrt{-}$
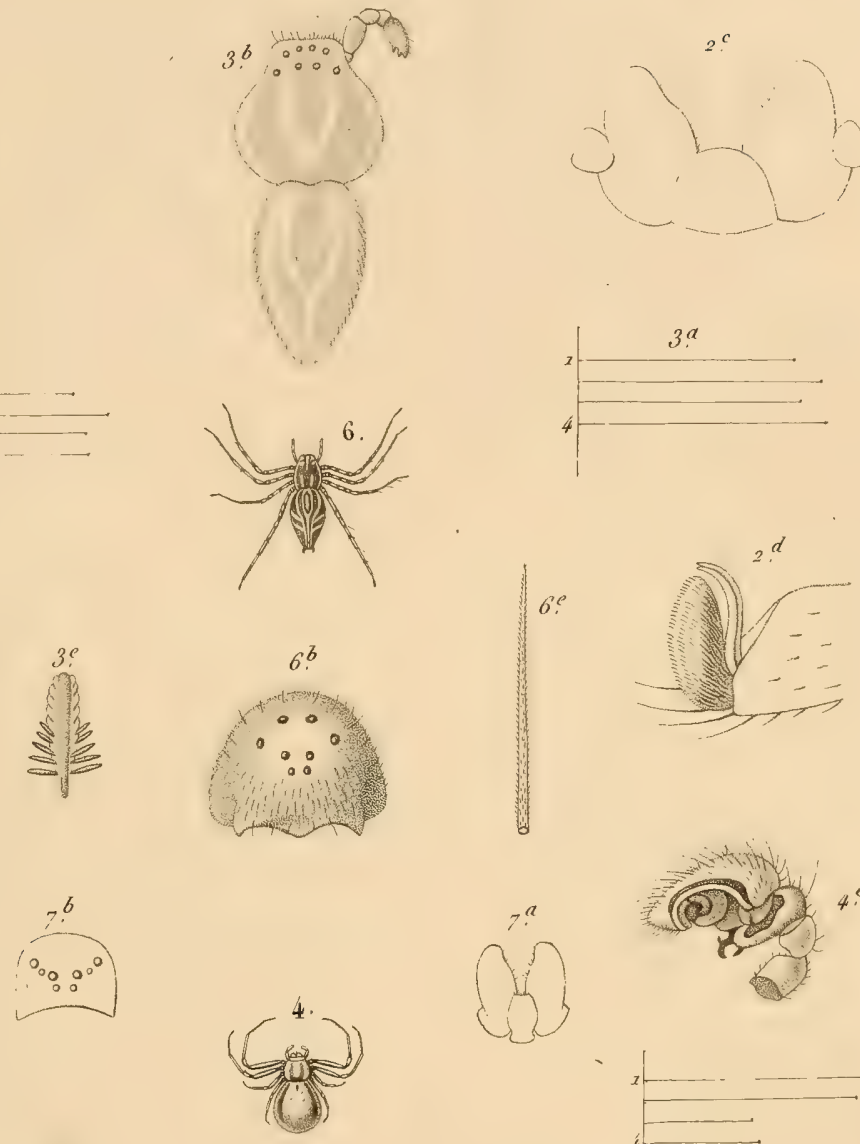
列
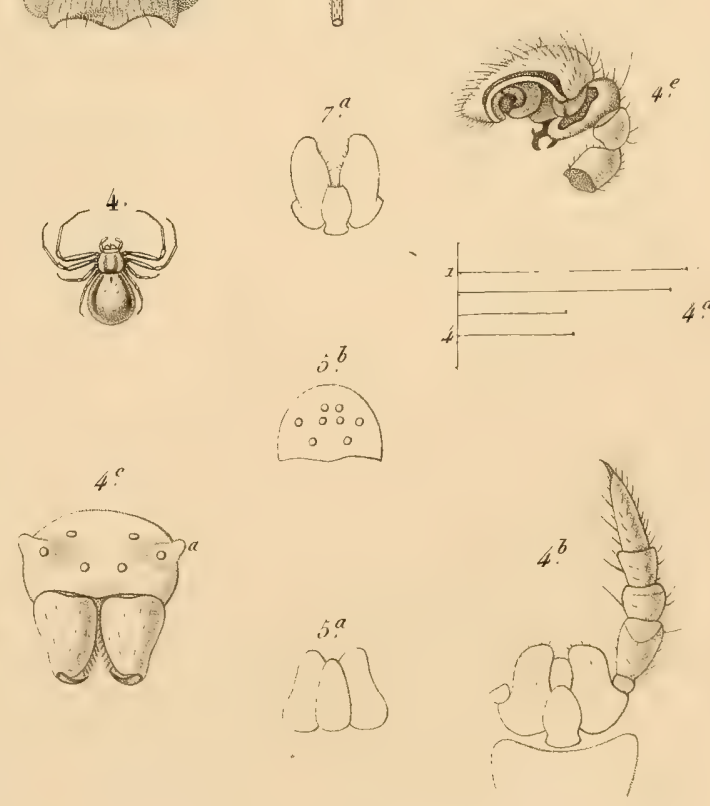

Girand sc.

Dugès del.

1. SÉLÉNOPE OMALOSOME. (Selenops omalosoma, Dhf.) 3.PHILODROME RHOMBIFERE (Philodromus shomboïcus, Walck,

4. THOMISE CITRON. (Thomisus citreus, Walck.) 6. OXIOPE PANACHÉ, (Oxiopes variegralus, Latr.) 



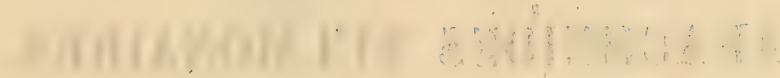

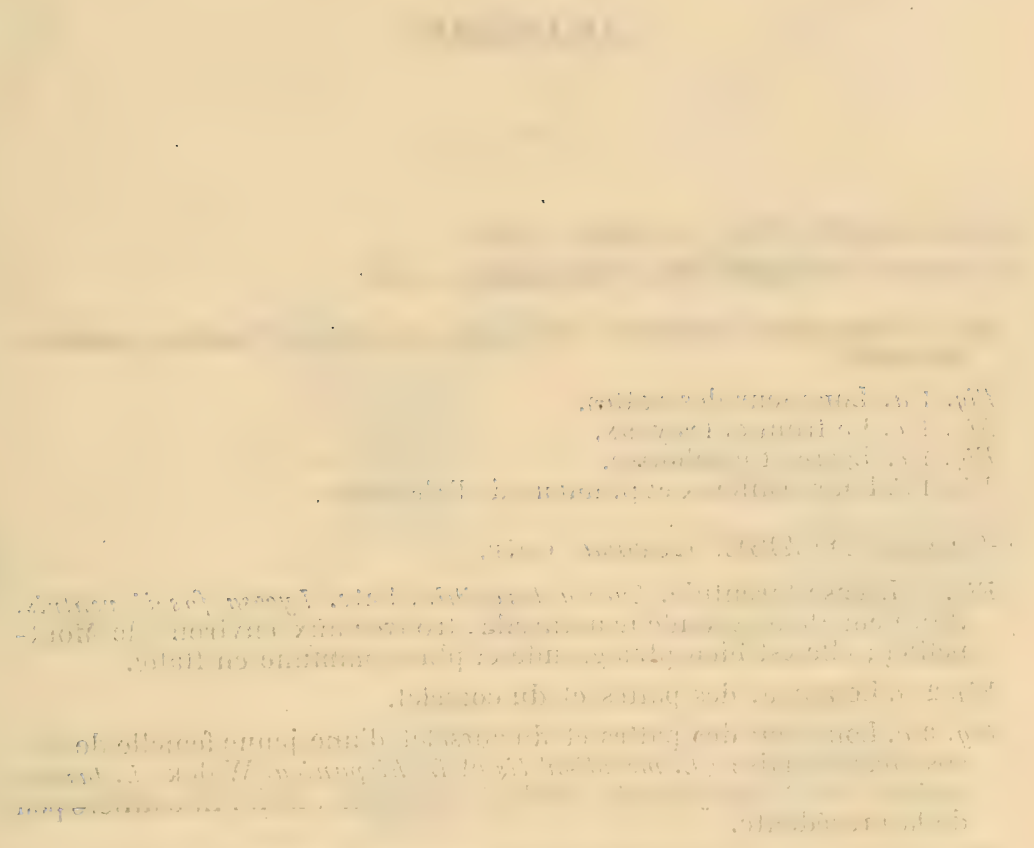

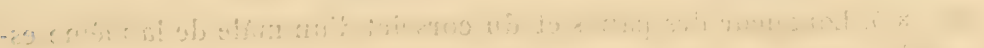

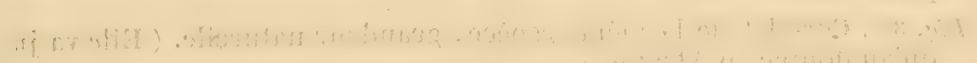

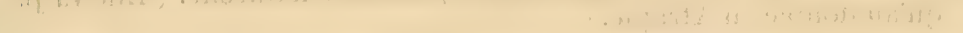

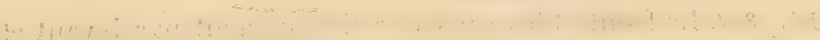

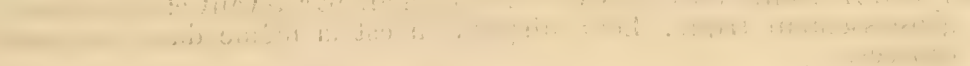




\title{
ARACHNIDES PULMONAIRES.
}

\author{
ARANÉIDES.
}

Genre ARATGNÉE. Aranea. Latr.

S.-Genre DOLOMEDE. Dolomedes. Latr.

Fig. 1. Dolomède admirable. Dolomedes mirabilis. Femelle, grandeur naturelle.

Fig. 1 a. Longueur des pattes.

Fig. $1 \mathrm{~b}$. Le front et les yeux.

Fig. 1 c. Lèvre et machoires.

Fig. $1 d$. Poils écailleux et plumeux de l'abdomen.

S.-Genre LYCOSE. Lycosa. Latr.

Fig. 2. Lycose tarentule. Lycosa tarentulu. Latr. Lycosa fascii ventris. Duf. Fem lle de grandeur naturelle, trouvée aux environs de Montpellier; elle est bien plus grande et plus commune en Italie.

Fig. 2 a. Longueur des pattes et du corselet.

Fig. $3 a$. Longueur des pattes et du corselet d'une jeune femelle de Lycose narbonnaise ( $L$. meridionnlis et $L$. hispanina. Walck. L. tarantulina. Sav. L. proegrandis. Koch. L. tarantula. Duf.). Eille diffère peu de la précédente.

Fig. 3 b. Longueur des pattes et du corselet d'un malle de la même espèce.

Fig. 3 c. Corselet de la même espèce, grandeur naturelle. ( Elle va jusqu'au double en A frique.)

Fig. $3 d$. Le front et les yeux vus obliquement par devant et d'en haut, grossissement triple. Leur disposition est la même dans les deux espèces.

Fig. 3 e. Lèvre et máchoire très grossies. $a$. Partie habituellement cachée sous les hanches antérieures.

Fig. $3 \mathrm{f}$. Tarse d'une patte antérieure avec griffes, ergot et brosses, pris sur une femelle; les dents sont plus nombreuses et plus longues dans le mâle.

Fig. 3 g. Un palpe du male de la L. narbonnaise.

Fig. $3 \mathrm{~h}$. Les trois sortes de poils qu'on trouve à l'abdomen.

S.-Genre MYRMÉCIE. Myrmecia. Latr.

Fig. 4. MYrmécie fauve. Myrmecia rufa. Male, double grandeur. D'après Latreille (Ann. Sc. nat.) aussi bien que les deux frgures suivantes.

Fig. $4 a$. Les yeux.

Fig. 4 b. La bouche avec un palpe. 


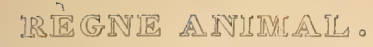

Qrachnides:

1113
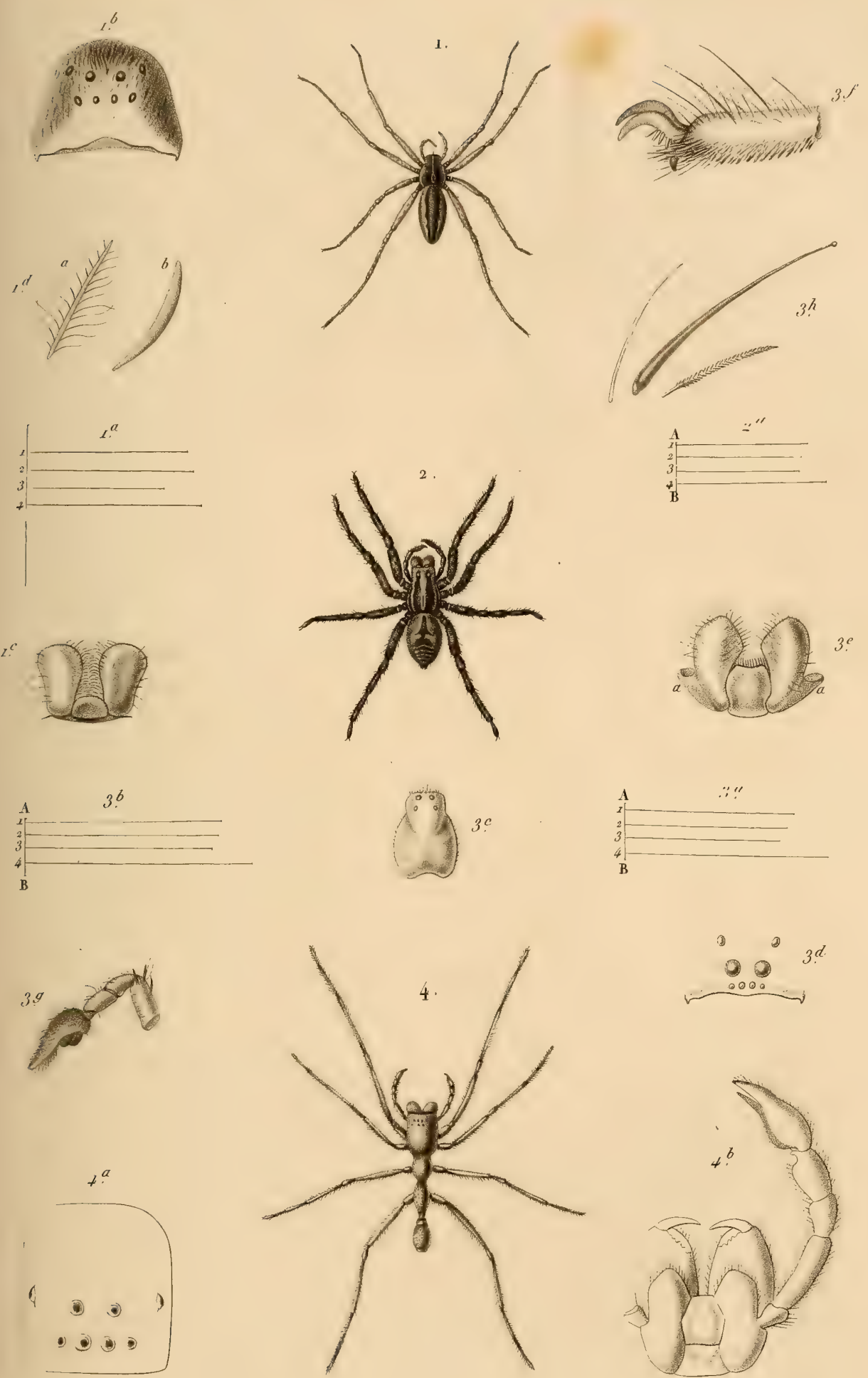

Ant.Dugèes del. 



\title{
ARACHNIDES PULMONAIRES.
}

\author{
ARANÉIDES.
}

Genre ARAIGNÉE. Aranea. Latr.

S.-Genre PALPIMANE. Palpimanus. Duf. Platyscelum. Sav. Chersis. Walck.

Fig. 1. Palpimane bossu. Palpimanus gibbulus. Duf. Femelle, un peu grossie. D'après Léon Dufour ainsi que les deux figures suivantes.

Fig. 1 a. Les yeux.

Fig. 1 b. Lèvre et máchoires avec un palpe.

Fig. 1 c. Les yeux d'un Platyscelum. D'après Savignny.

S.-Genre ERESE. Eresus. Walck.

Fig. 2. ÉRÈse cinabRe. Eresus cinnabariım. Mâle de grandeur naturelle.

Fig. $2 a$. Palpe très grossi, vu par dehors.

Fig. 2 b. Poil plumeux pris en dessus de l'abdomen.

Fig. 3 a. Le front et les yeux, vus obliquement par devant et en dessus, dans l'Érèse noir (Eresus niger. Latr. Er. frontalis. Walck. Er. imperialis. Duf. Er. petagna. Sav.). Grossissement plus que triple.

Fig. 3 b. Lèvre, máchoires et plastron du même.

S.-Genre SALTIQUE. Salticus. Latr. Attus. Walck.

Fig. 4. Saltique Ghevronné. S. scenicus. Femelle, grandeur doublée.

Fig. 4 a. Longneur des pattes quadruplée. Les premières sont les plus longues dans le male.

Fig. 4 b. Le front et les yeux, vus d'en haut et par devant.

Fig. 4 c. Bouche de la femelle avec un palpe.

Fig. $4 d$. Bout d'une patte montrant une des deux griffes et une des deux houpes. L'antre griffe cachée est plus denticulée que celle-ci.

Fig. 4 e. Une des écailles qui couvrent le corps.

Fig. $4 f$. Un palpe male.

Fig. $4 \mathrm{~g}$. Une mandibule du mâle avec une mâchoire et la lèvre.

S.-Genre ENYO. Sav. réuni au genre Clotho par Walck.

Fig. 5. Enyo occitanica, nob. Grandeur naturelle.

Fig. 5 a. Le corps très grossi. On peut y voir les yeux disposés com me chez les Clothos, et les filières saillantes de même, mais le reste est tout différent.

Fig. 5 b. Longueur des pattes doublée.

Fig. 5 c. Les yeux.

Fig. 5 d. La bouche et le plastron.

Fig. 5 e. Bout d'une patte; un article supplémentaire et des soies crćnelées comme chez les Scythodes.

Fig. $5 \mathrm{f}$. Poils de l'abdomen. Le tout d'après nature.

S.-Genre LACHESIS. Sav. Voisin du précédent, peut-être identique.

Fig. 6 a. Bouche du LACHESIS PERVERSA. Lachesis perversa. Les crochets des mandibules n'ont paru sans doute courbés en avant qu'à cause de l'inclinaison sous laquelle la tête a été présentée au dessinateur. Figure empruntée, comme les huit suivantes, à la description de l'Egypte.

Fig. 6 b. Les yeux de la mème aranéide.

S.Gerre HERSILIE. Hersilia. Sav. Voisin des Araneas.

Fiy. 7 a. Bouche de l'Hersilia caudata.

Fig. 7 b. Les yeux et le front.

Fì. 7 c. Extrémité de l'abdomen avec ses filières.

S.-Genre ERYGONE. Sav. Voisin des Théridions.

Fig. $8 a$. Bouche de l'Érygone vagans.

Fig. 8 b. Les yeux.

Fiy. $8 c$. Une des mandibules, vue de proili.

Fig. $8 d$. Le trone du mile avec un palpe. 


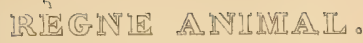

Qrochnides.

P1, 14
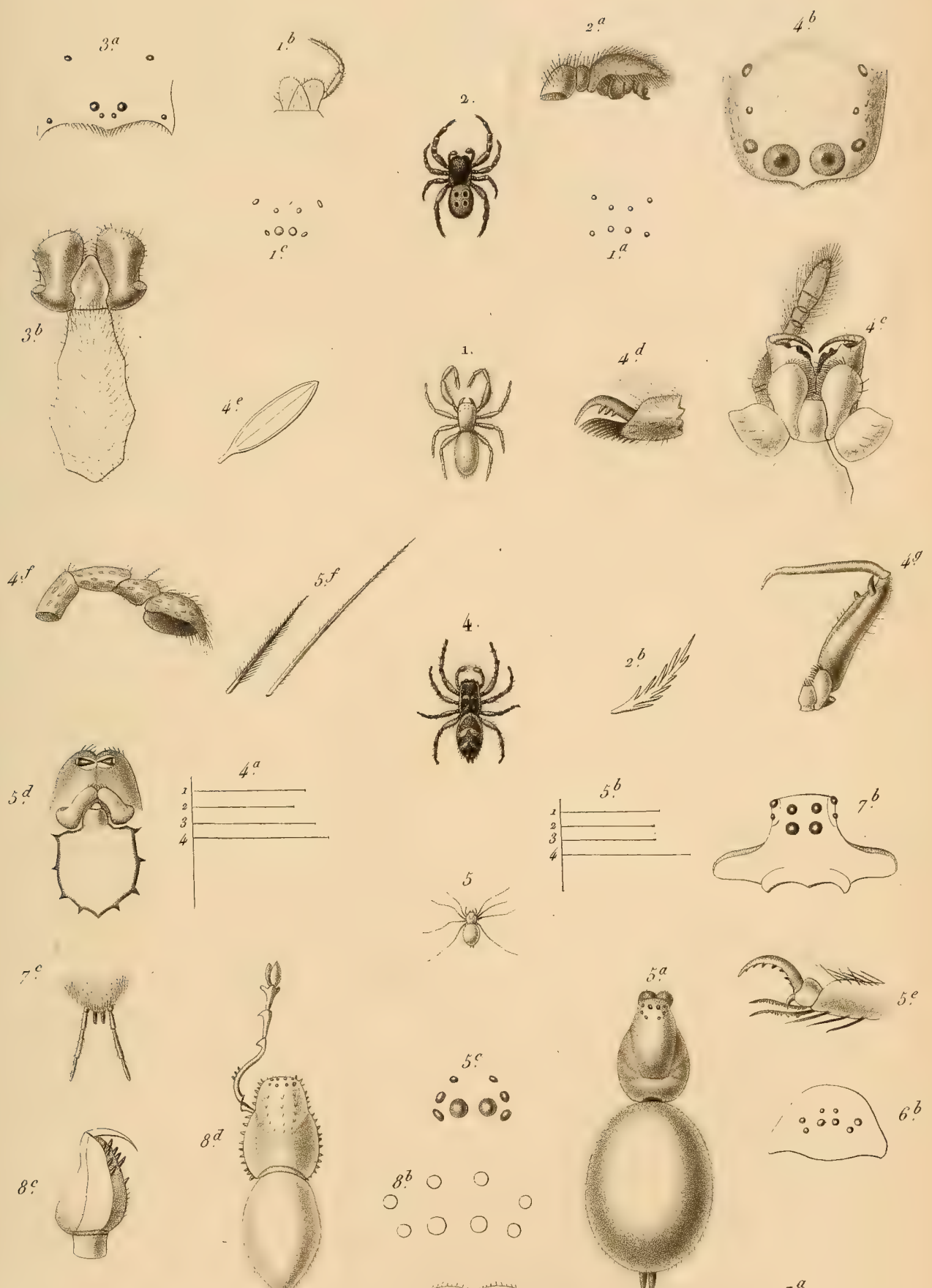

5

10
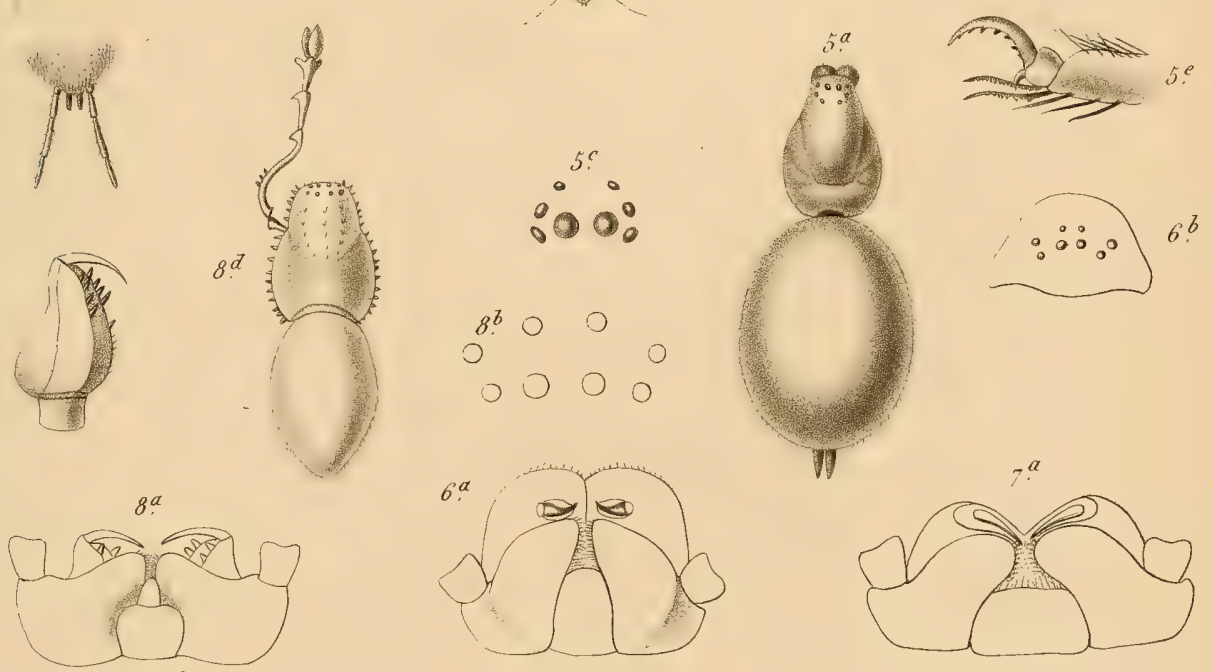

Ant. Dugiès del.

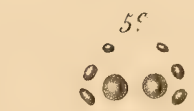

1. PALPIMANE BOSSU: (Palpimanus gibbulus.)

2. ERE'SE CINABRE. (Eresus cinnaberinis.) 




\section{ARACHNIDES PULMONAIRES.}

Supplẹment aux figures relatives à la famille des FILEUSES.

GenRe SPIIODROS. Sphodros. Walck.

Fig. 1. Yeux du Sphodros Milberti. Walck.

S.-Genre UP'TIOTE. Uptiotes. Walck.

Fig. 2. Yeux de l'vptiote incertaine. Uptiotes anceps. Walck.

Fig. 2 a. Bouche de la inême.

S.-Genre DOLOPHONE. Dolophones. Walck.

Fig. 3. Yeux du Dolophone Notacantre. Dolophones notacantha. Walck. Aranea notacantha. Quoy et Gaimard.

Genre DELÈNE. Delena. Walck.

Fig. 4. Yeux du Delène péronien. Delena peronianus. Walck.

Fig. 4 a. Bouche du même.

S.-Genre CLASTÈS. Clastes. Walck.

Fig. 5. Corps du ci.Astès Freycinet. Clastes Freycinetii. Walck.

Fig. 5 a. Yeux du même.

Fig. 5 b. Bouche du même.

S.-Genre DESIS. Desis. Walck.

Fig. 6. Yeux du Desis DYsDérö̈des. Desis dysderoìdes. Walck.

Fig. 6 a. Bouche du mème.

S.-Genre ARTEMME. Artema. Walck.

Fig. 7. Yeux de l'artème madricienne. Artema mauritiana: Walck.

Fig. 7 a. Bouche du même.

S.-GFnRe MICRYPHANTE. Micryphantes.

Fig. 8. Corps du micryphantes camelinus. Koch.

Fig. $8 a$. Corselet de la femelle, vu de profil.

S.-Genre EPISINE. Episinus. Walck.

Fig. 9. Bouche de l'episinus truncatus.

Fig. 9 a. Tête du même.

S.-Genre ARGYRONECTE. Argyronectes.

Fig. 10. ARGYRONECTE AQUATIQUe.

Fig. $10 a$. Yeux du même.

FAMILLE DES PÉDIPALPES. Pedipalpi.

Genre TARENTULE. Tarantula. Fabricius.

S.-Genre THÉLYPHONE. Thelyphonus. Latr.

Fig. 11. ThÉLYPhone A QUEUe. Thelyphonus caudatus. Latr. Phalangium caudatum. Lin. Grandeur naturelle.

Fig. $11 a$. Le tronc, vu en dessous et grossi au double.

Fig. 11 b. Corselet, vu de cóté.

Fig. $11 c$. Mandibule, grossie:

Fig. 11 d. Une pince, grossie.

Fig. 11 e. Un tarse, très grossi. 
R GNIEANIMLAID

Aracbitides.

P1. 15 ,

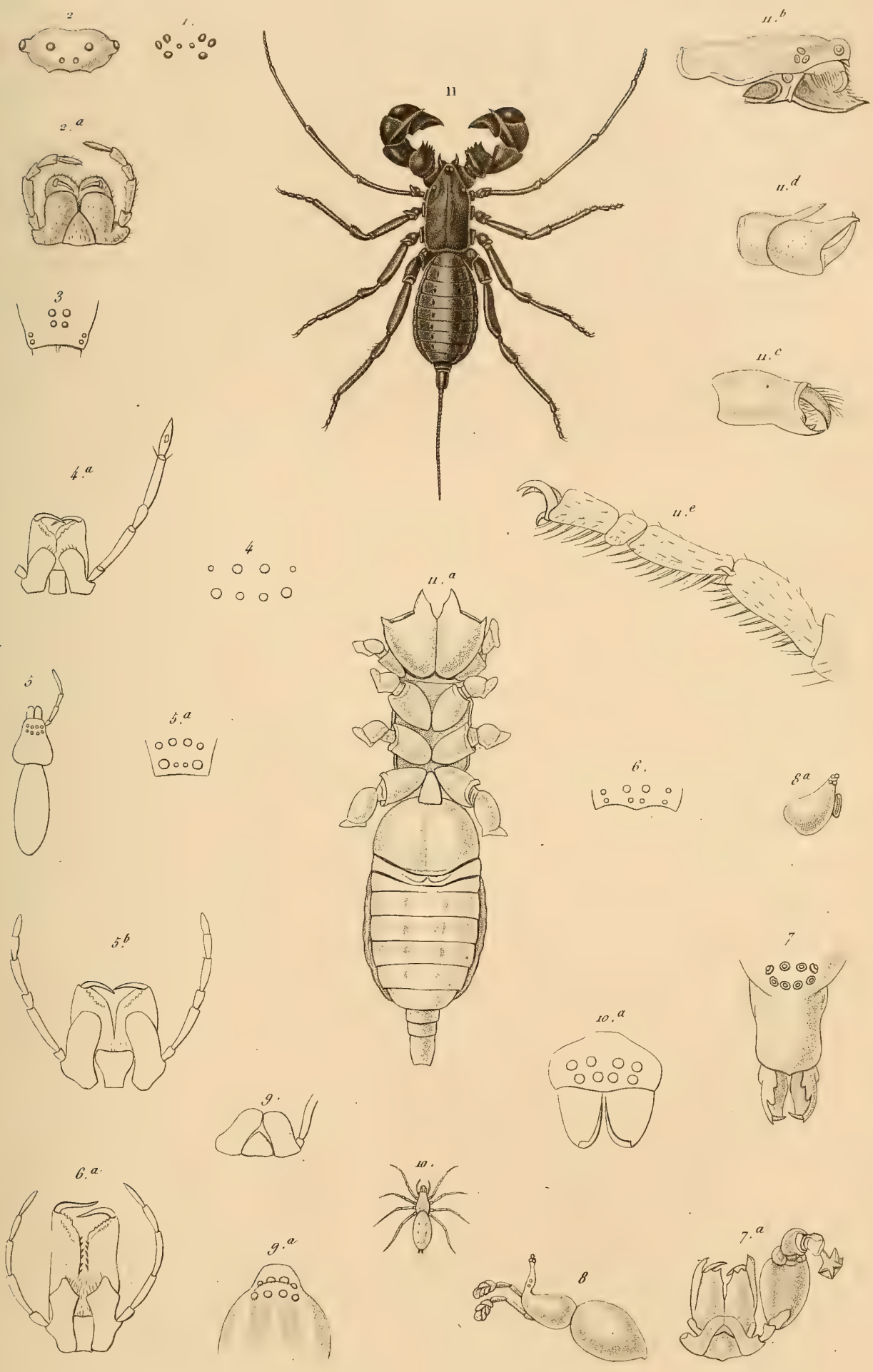

11. THÉLYPHONE A QUEUE, (Thelyphonus caudatus) 




\title{
ARACHNIDES PULMONAIRES.
}

\author{
PÉDIP ALPES.
}

Genre TARentule. Tarantula. Fabr.

S.-Genre PhRYNE. Phrynus. Olivier.

Fig. 1. Phryne réniforme. Phrynus reniformis. Grandeur naturelle.

Fig. 1 a. Portion antérieure du corps, vue en dessous et grossie du double.

Fig. 1 b. Portion antérieure de la carapace; même grossissement.

Fig. 1 c. La languette, très grossie.

Fig. 1 d. Mandibule; grossissement de deux.

Fig. 1 e. La mème déponillée de ses poils et grossie davantage.

Fig. $1 f$. Les trois derniers articles d'un tarse. 

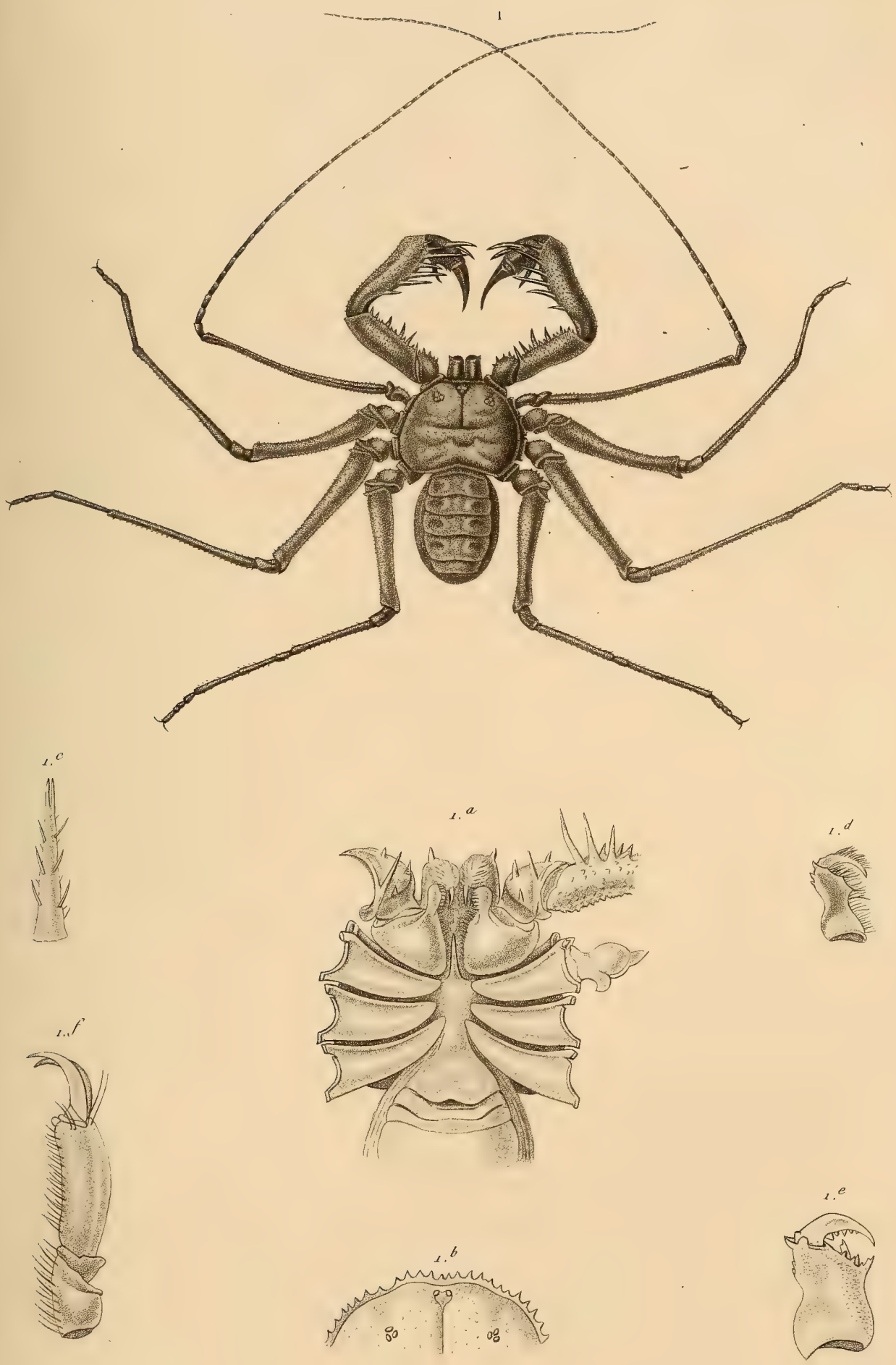

1. PHRYNE RENIFORME, ( Phrynus reniformis.) 




\title{
ARACHNIDES PULMONAIRES.
}

\author{
PEDIPALPES.
}

Genre SCORPION. Scorpio. Lin.

Fig. 1. Scorpion d'Afrieque. Scorpio Afer. Lin. Degrandeur natureile.

Fig. 1 a. Céphalo-thorax du même grossi pour montrer les yeux.

a. Premier anneau de l'abdomen. - b. Céphale-thorax - co Yeux médiodorsaux. - $d$. Les trois paires d'yeux latéraux.

Fig. 1. b. Portion postérieure de l'abdomen, vue de côté.

$a$. Dernier article terniné en crochet. $-b$. L'un des orifices de rappareil ver nimeux.

Fig. 1 c. Extrémité de l'une des antennes-pinces.

Fig. 1 d. Extrémité de l'un des palpes.

Fig. 1 e. Extrémilé de l'une des pattes postérieures, vue en dessous.

Fig. I f. La mème partie, vue en dessus.

$$
\text { (D'après nature.) }
$$




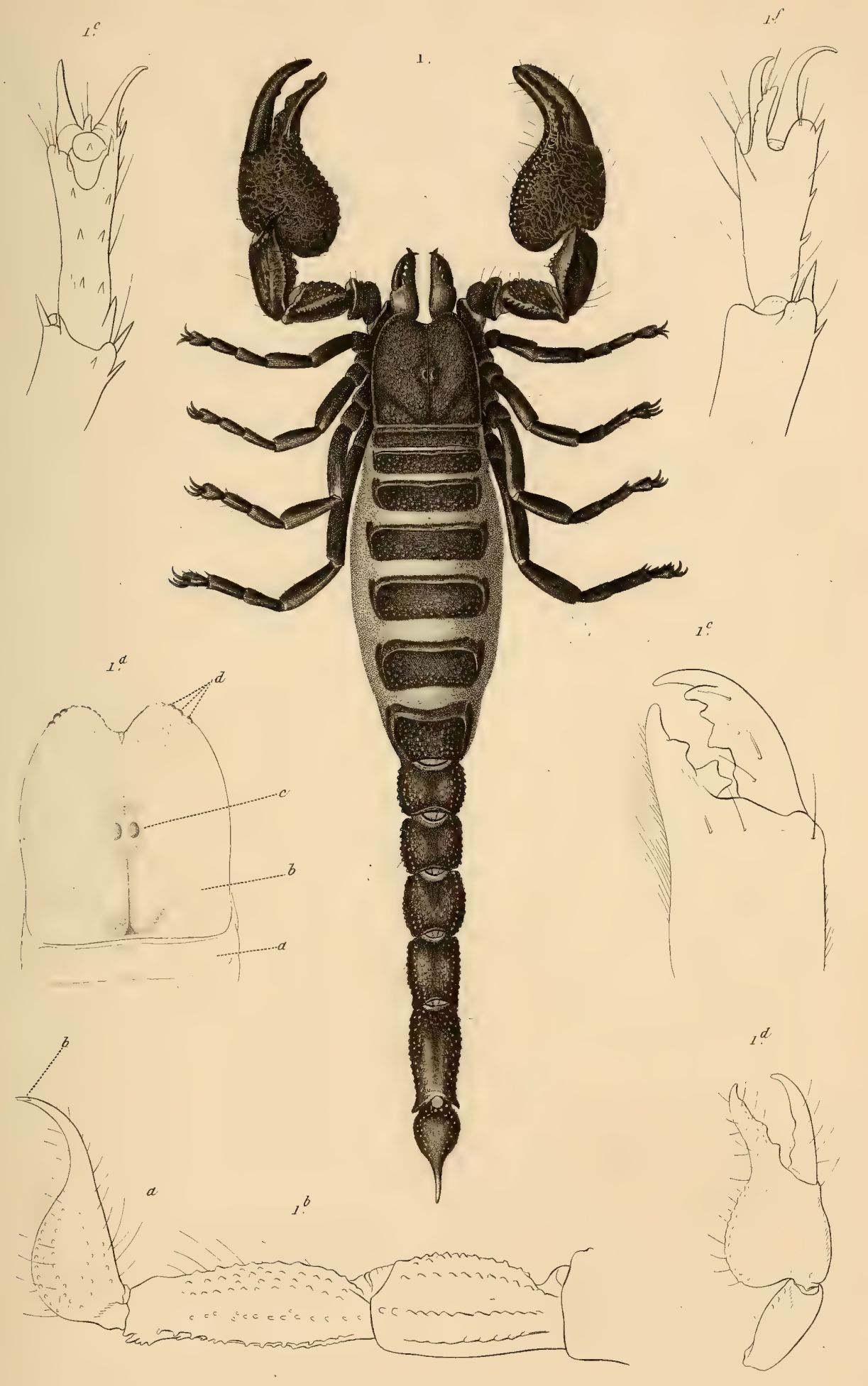






\title{
ARACHNIDES PULMONAIRES.
}

\author{
PEDIPALPES.
}

\section{ORGANISATION DES SCORPIONS.}

Fig. 1. Thorax et portion antérieure de l'abdomen du Sconprox b'dFriQUE, vue en dessous.

$a$. Antennes-pinces. $-b$. Base des palpes. - $c, d, e, f$. Base des pattes. - g. Lame operculaire recouvrant l'orifice de l'appareil reproducteur. $-k$. Peigne. $-i, i$. Les quatre paires de stigmates.

Fig. 1 a. Extrémité antérieure du corps, vue par devant.

$a$ Antennes-pinces. $-b$. Base des palpes. $-c$. Base des pattes antórieures. d. Appendices basilaires des pattes de la seconde paire.

Fig. 1 b. Pattes de la première paire.

Fig. 1 c. Patte de la seconde paire.

Fig. 1 d. Région génitale.

$a$. Thorax. $-b$. Opercule génital. - $c$. Orifice de l'appareil géuital. $-d, d$. Peignes.

Fig. 1 e. Extrémité de l'abdomen, vue en dessous pour montrer la position de l'anus $(\boldsymbol{a})$.

Fig. $1 f$. L'une des poches pulmonaires, vue par sa face supérieure et fortement grossie.

Fig. 1 g. Quelques-unes des lames respiratoires extraites de la poche précédente et grossies davantage.

( $D^{\prime}$ après nature.) 


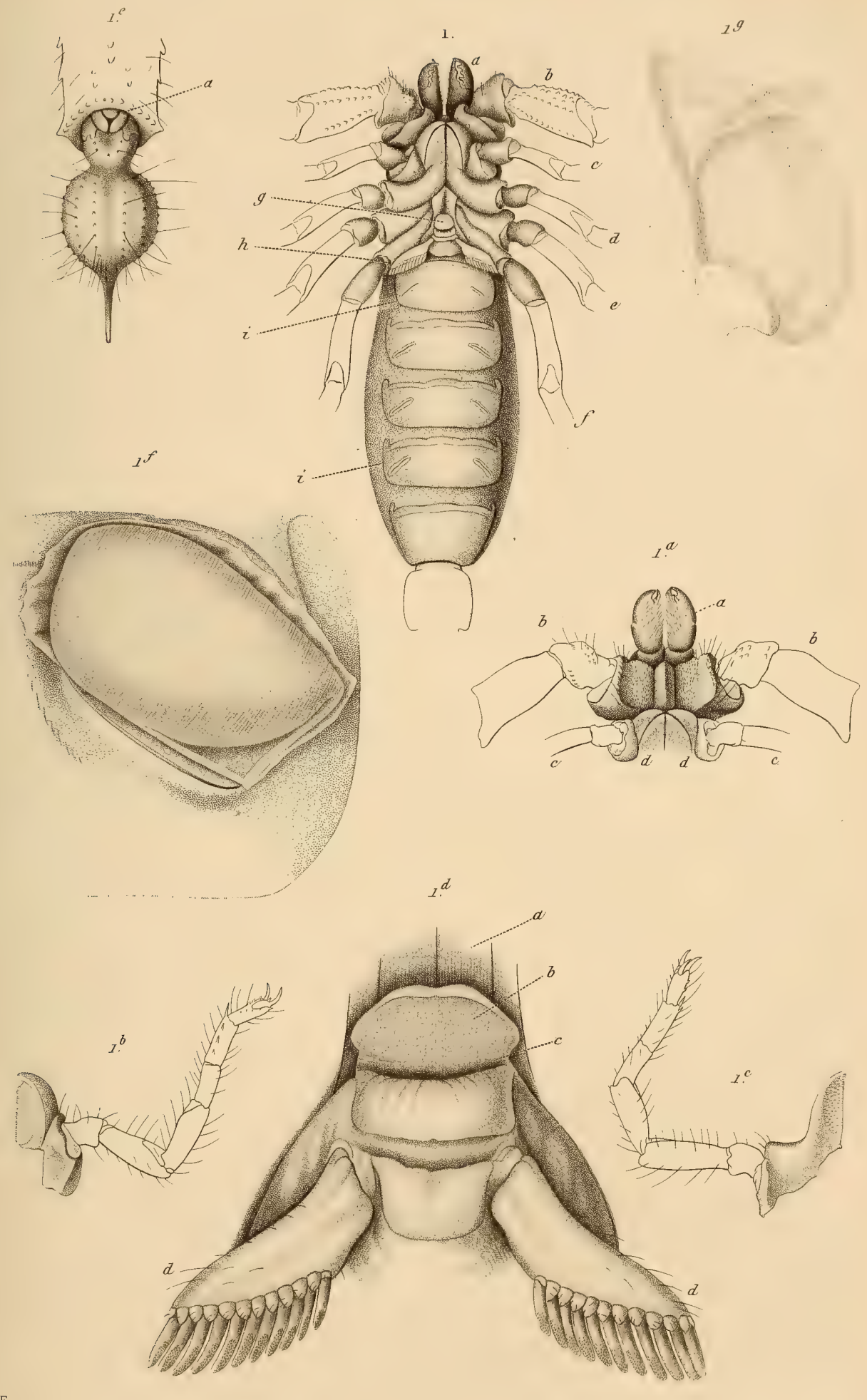





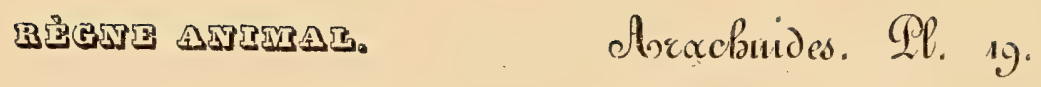

\title{
ARACHNIDES PULMONAIRES.
}

\author{
PÉDIPALPES.
}

GeNRe SCORPION. Scorpio. Lin.

Fig. 1. Scorpion roussatre. Scorpio oceitaneus, L. Buthus oceitaneus. Leach. Grossi au double et vu en dessus.

Fig. 1 a. Le même en dessous.

a. Bouche. $-b$. Base des palpes. - $c, d, e, f$. Portion basilaire des pattes. g. Pièces sternales des pattes des deux premières paires simulant une sorte de lèvre inférieure. - $i$. Orifice sexuel. $-h$. Appendices en forme de peignes. $-i$. Stigmates de la seconde paire. $-k$. Stigmates de la quatrième paire. - $l$. Sixième anneau abdominal. - $m$. Septième anneau abdominal.

Fig. 2 Scorpion D'Europe. Scorpio europeus. L. De grandeur naturelle. Fig. 2 a. Céphalo-thorax du même grossi pour montrer les six yeux qui sont caractéristiques du genre Scorpion proprement dit de Leach.

$a$. Les deux yeux médio-postérieurs. - $b$. Les yeux latéro-antérieurs.

Fig. 2 b. Orifice sexuel et peignes du même, beaucoup grossi.

$a$. Base du thorax. - b. Base des pattes de la troisième paire. $-c$. Plaques operculaires de l'orifice sexuel. $-d$. Abdomen. $-e$. Peigue.

Fig. 2 c. Extrémité de l'une des paltes.

Fig. 3. Yeux du Scorpion palyé. Buthus palmatus. Ehrenb. Espèce qui a, à raison de la petitesse des yeux de la trossième paire latérale, tend à établir le passage entre les Scorpions proprement dits et le genre Buthus de Leach. D'après M. Ehrenberg.

Fig. 4. Yeux d'un Scorpion du genre Androctonus chez lequel ces organes sont au nombre de dix, caractère qui distingue ce groupe. D'après M. Ehrenberg.

Toutes ces figures, excepté les deux dernières, sont d'après nature. 


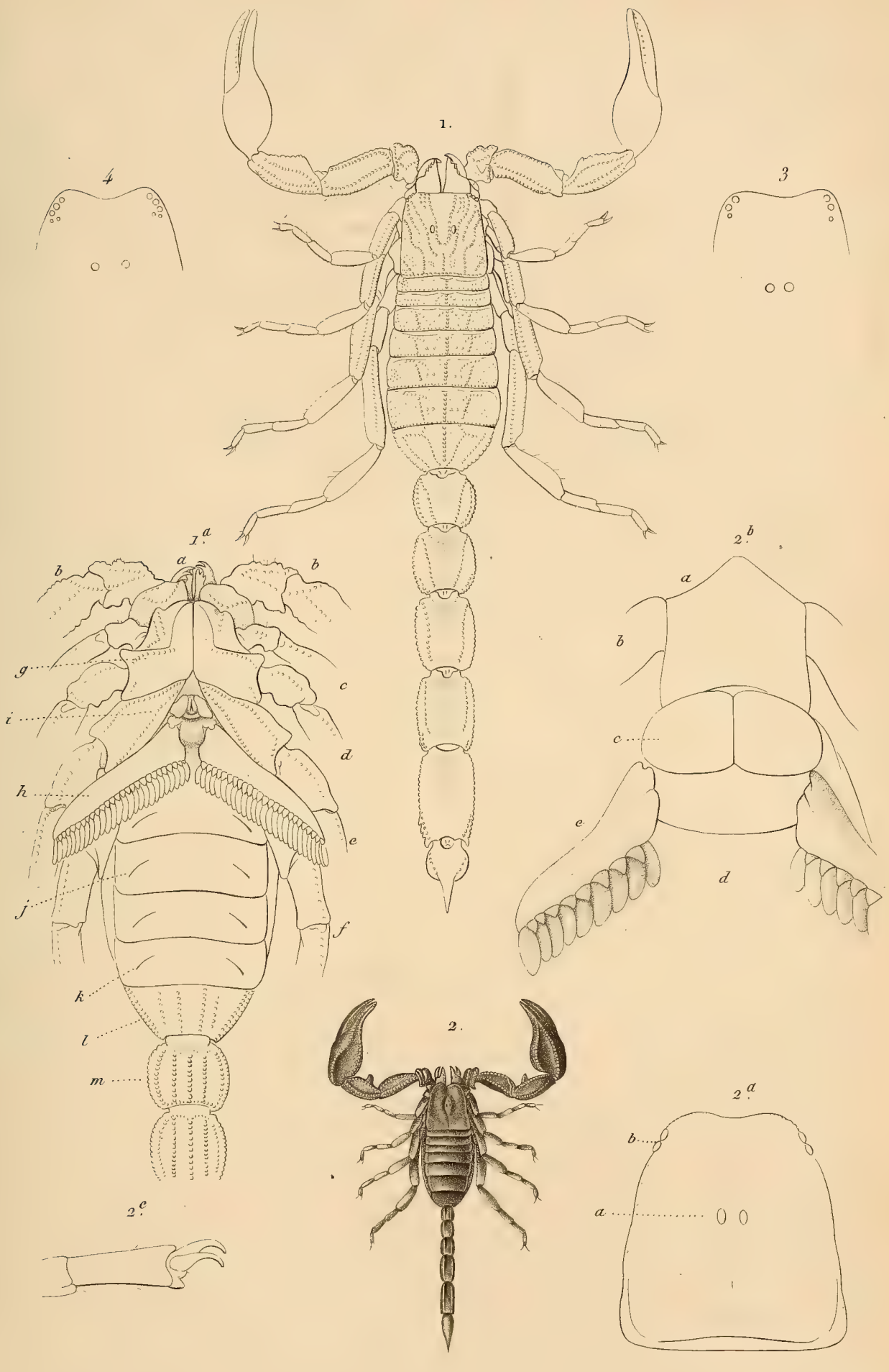

1. SCORPION ROUSSÄTRE. (Scorpio occitaneus.) 




\title{
ARACHNIDES PULMONAIRES.
}

\author{
PEDIPALPES.
}

\section{ANATOMIE DES SCORPIONS.}

Fig. 1. Système nerveux d'un ANDroctone, d'après M. Newport (On the structure, elc., of the nervous and eirculatory systems, Phil. trans. 1843 ).

$a$. Les ganglions cérébroïdes ou céphaliques (cerveau Latr.). $-b$. Les yeux principaux et leurs nerfs optiques. $-c$. Les yeux latéraux et les nerfs optiques acces* soires. $-d$. Nerfs antennaires se rendant aux antennes-pinces. $-e_{0}$. Masse nerveuse formée par la réunion des ganglions postœsophagiens de toute la portion antérieure du corps. - f. Nerfs des palpes pédiformes. - $g$. Nerfs des pattes de la première paire. - $h$. Nerfs des pattes de la quatrième paire. - $i$. Nerfs des premiers anneaux de l'abdomen naissant du bord postérieur de la masse ganglionaire thoracique. - $j$. Nerfs dont quelques branches se rendent au sac pulmonaire de la seconde paire. - $k$. Ganglion correspondant à l'anneau abdominal qui porte les saes brauchiaux de la troisième paire. - $l$. Ganglion de l'antépénultième segment de l'abdomen, $-m$. Nerfs de l'anneau anal, $-n$. Nerfs de l'annean terminal quỉ renferme l'appareil renimeux. 


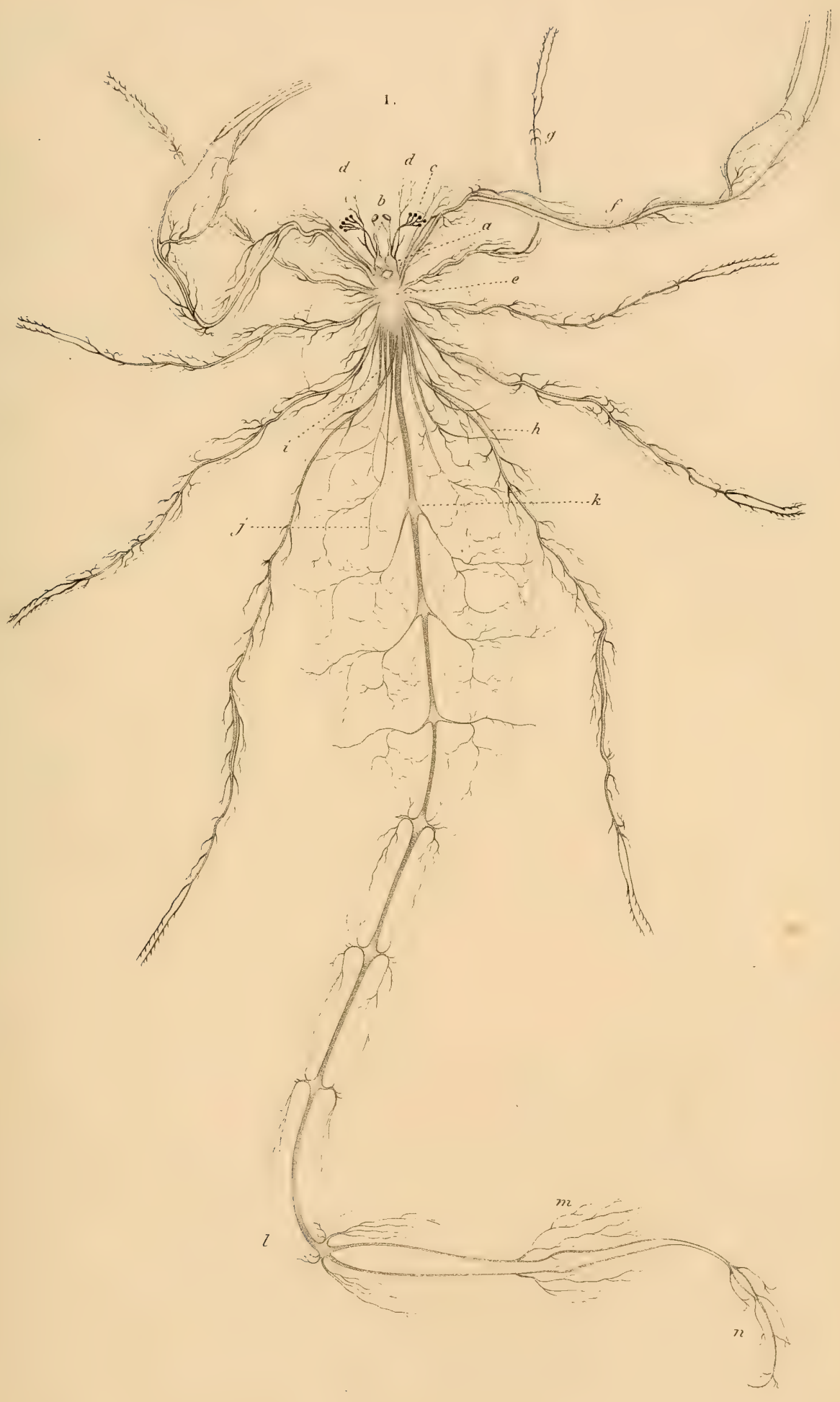

ORGANTSATIOV DES SCORPIONS 




\title{
ARACHNIDES PULMONAIRES.
}

\author{
PEDIPALPES.
}

\section{ANATOMIE DES SCORPIONS.}

Fig. 1. Appareil vasculaire d'un Scorpion. Buthus afer. Vu en dessus, d'après M. Newport (op. cit.).

a. Portion ventriculaire du vaisseau dorsal divisée en une série de loges, $-b$. Première paire des ailes musculaires du vaisseau dorsal. $-c, c$. Branches artérielles latérales se distribuant dans chaque anneau aux muscles et aux viscères. $-d$. Portion abdominale du vaisseau dorsal. $-e$. Vaisseaux pneumocardiaques. $-f$. Portion antérieure du vaisseau dorsal ou artère-aorte donnant naissance aux artères ophthalmiques, maxillaires, pédicuses, etc. (Voyez les deux figures suivantes):

Fig. 2. Figure théorique de l'appareil de la circulation chez le Buthus a fer. Grossi (d'après le méme).

a. Le cœur ou portion ventriculaire du vaisseau dorsal divisé en sept loges; $a^{\prime}$. Orifices latéraux par lesquels le sang pénètre dans les ventricules. $-b$. Portion postérieure du vaisseau dorsal ou artère abdominale. - $c$. Portion antérieure ou aortique du vaisseau dorsal qui pénètre dans le céphalo-thorax. - $d$. Artères céphaliques qui naissent de l'extrémité antérieure de l'aorte, contournent les ganglions cérébroïdes, puis se dirigent en arrière et envoient des branches aux glandes salivaires, aux muscles de la face dorsale du corps, ete. - $e, e$. Artères ophthalmiques. - $f$. Artères des antennes-pinces. $-g$. Artères des palpes. $-h$. Artères des pattes antérieurs; $h^{\prime}$. Artères des pattes postérieures. $-j$. Artère sternale provenant de l'extrémité antérieure de l'aorte, et s'avançant sous la chaine ganglionaire $k$ jusqu'à l'extrémité postérieure du corps.- $l$. Branches latérales du vaisseau dorsal,- $m$. Artère gastrique naissant de l'aorte.- $n$. Artère récurrent qui nait également de l'aorte et qui longe la face supéricure de la chaíne ganglionaire. - 0 . Dernière branche pneumocardiaque. - $p, p$. Le canal digestif avec une portion de ses appendices. $-q$. Terminaison des canaux biliaires. $-r$. $r$. Poches pulmonaires.

Fig. 3. Portion antérieure de l'aorte et appareil digestif, grossis (d'après le même).

a. L'estomac. - $b$. Intestin.- $c$. Anus.- $d$, $d$. Appendices gastriques et corps adipeux. - $e$. Portion des canaux biliaires. $-f$. Portion terminale de l'aorte.$g$. Artère gastrique. $-h$. Artèrés des pattes, etc- $i$. $i$. Artères céphaliques. 

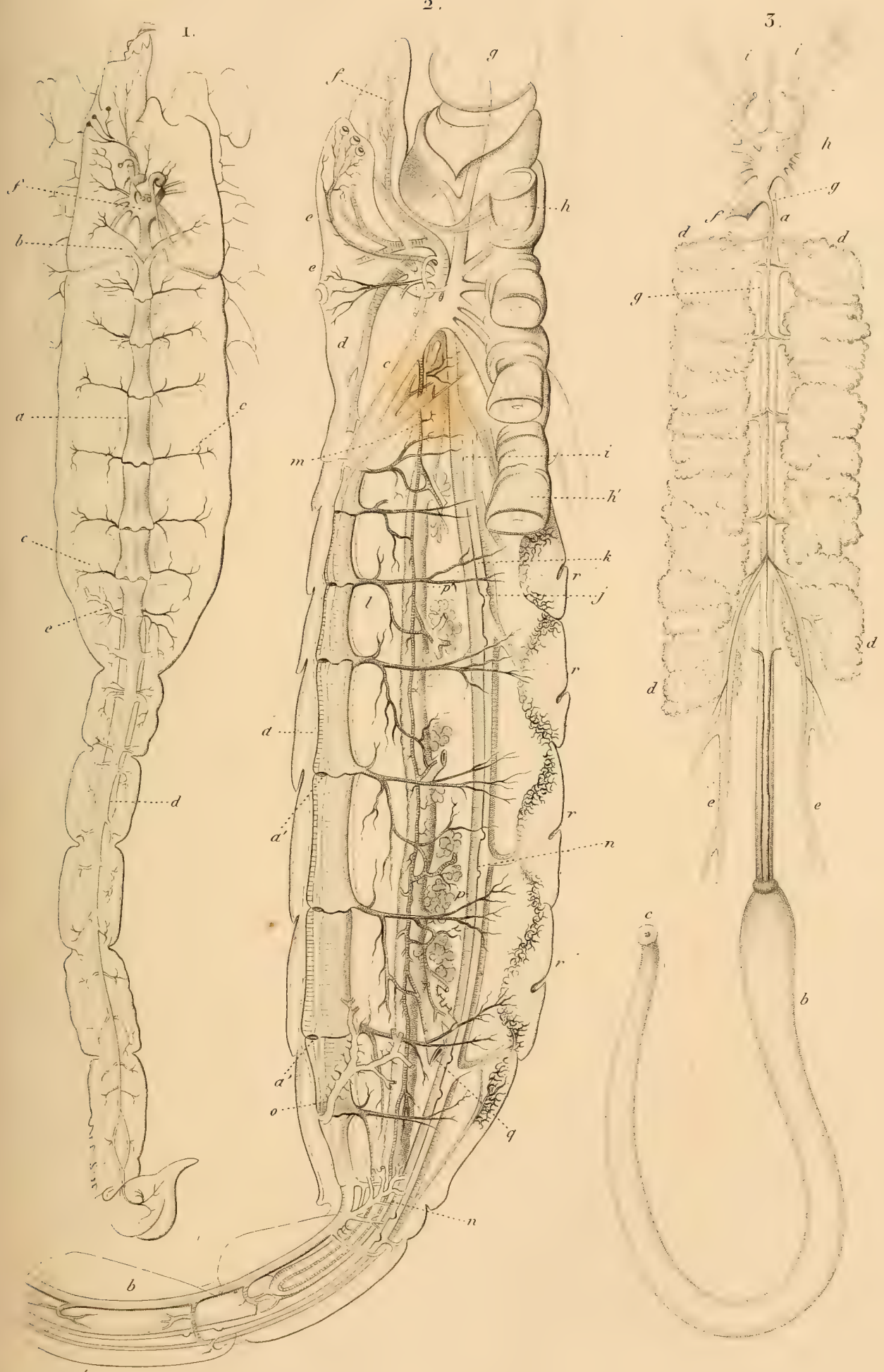

ORGANISATTON DES SCORPIONS 



\title{
ARACHNIDES TRACHÉENNES.
}

\author{
FAUX SCORPIONS.
}

Genne GALÉODE. Galeodes. Olivier, Latreille; Solpuga. Lichtenstein, Walckenaer, etc.

Fig. 1. Galéode araIgnéE. Galeodes araneoides. Oliv. De grandeur naturelle.

Fig. 1 a. Tubercule du bord antérieur du céphalo-thorax portant les yeux.

Fig. 1 b. Portion antérieure du corps, vue de profil.

$a$. Céphalo-thorax. - $b$. Yeux. $-0, d$, e. Les trois derniers anneaux du thorax portant les trois dernières paires de pattes. $-f$. Antennes-pinces; $f$. Appendice palpiforme de ces organes. $-g$. Appareil buccal. $-h$. Bases des palpes pédiformes. - $i$. Base des pattes de la première paire.

Fig. 1 c. Antennes-pinces, vues de profil.

a. Le petit appendice qui naît sur ces organes chez le mâte.

Fig. $1 d$. Extrémité de l'un des palpes.

Fig. 1 e. Extrémité de l'une des pattes de la première paire.

Fig. $1 f$. Extrémité de l'une des pattes suivantes.

(Les quatre dernières figures sont reproduites d'après les planches de M. Savigny; les trois premières sont d'après nature.) 


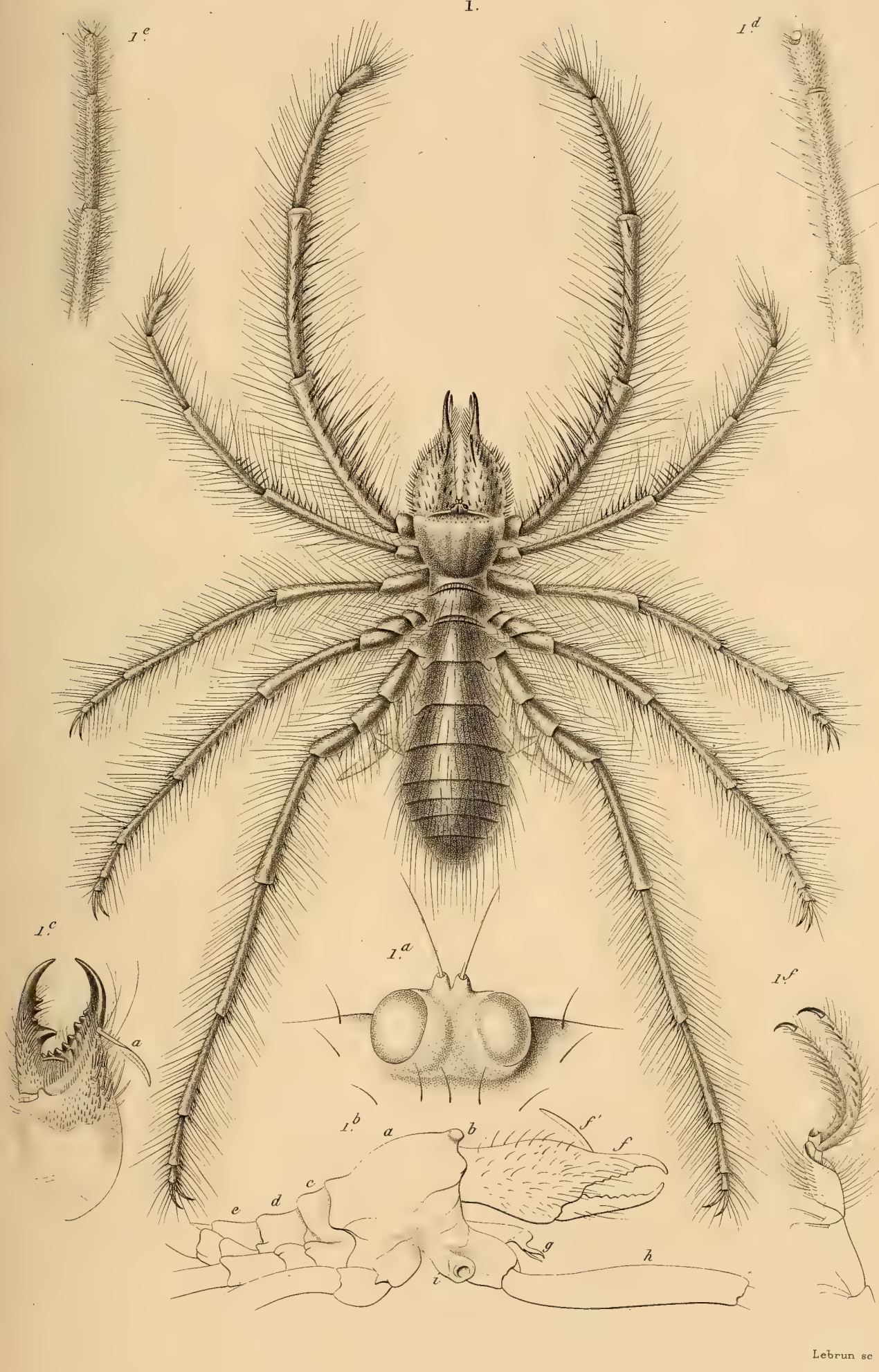

GALÉODE ARATGNEE. (Galeodes araneoïdes.) 




\title{
ARACHNIDES TRACHÉENNES.
}

\author{
FAUX SCORPIONS.
}

\section{Genre GalÉODE. Galeodes. Oliv. Solpuga. Iicht.}

Fig. 1. Galéode araignée. Galeodes araneoides. Vu de profil d'après M. Savigny (grand ouvrage sur l'Egypte).

Fig. 1 a. Appareil buccal, et base des palpes et des pattes de la première paire (d'après le mème).

Fig. 1 b. Appareil buccal, vu de profil, et composé de pinces qui représentent les mandibules et les mâchoires (d'après le même).

Fig. 2. Face inférieure du corps de la Galéode de Barbarie. Galeodes barbara. Lucas. Grossie (d'après un dessin inédit de M. Milne Edwards ).

a. Appareil buccal. - $b$. Antennes-pinces. $-c$. Base des palpes pédiformes. $-d$. Orifices respirateurs situés derrière la base des pattes de la seconde paire.-e. Portion des pattes de la dernière paire, garnies des appendices spatuliformes, $g$. Orifice génital.- $h, i$. Orifices stigmatıques situés sous le bord postérieur du second et du troisième anneau de l'abdomen et conduisant l'air dans les trachées. $-j$. Anus.

Fig. $2 a$. L'un des stigmates abdominaux (d'après nature).

$a$. Bord postérieur de l'anneau- $b$. Épines disposées en dents de peigne sous ce bord.- - c. Stipule.

Genre PINCE. Chelifer. Geoffroy.

\section{S.-Genre CHELIFER proprement dit. Chelifer. Leach.}

Fig. 3. Chellfer de Bravais. Chelifer Bravaisii. Gervais. Beaucoup grossi (d'après nature).

Fiy. 4. Céphalo-thorax du Chelifer sésamoïde pour montrer la disposition des yeux (d'après M. Savigny, op. cit.).

Fig. 4 a. Base des pattes et région buccale du même (d'après M. Savigny).

Fig. 4 b. Appareil buccal (d'après le même).

Fig. 4 c. Antenne-pince.

S.-Genre OBISIE. Obisium. Leach.

Fig. 5. Obisie trombidioüde. Obisium trombidioides. Latr. Chelifer ischnocheles. Herman. Grossi (d'après de Theis, Ann. des Sc. Nat., $1^{\text {re }}$ série, t. XXVII ).

Fig. 5 a. Région buccale du même, vue en dessous.

Fig. 5 b. Céphalo-thorax et antennes-pinces du mème pour montrer la disposition des yeux (d'après le mème). 

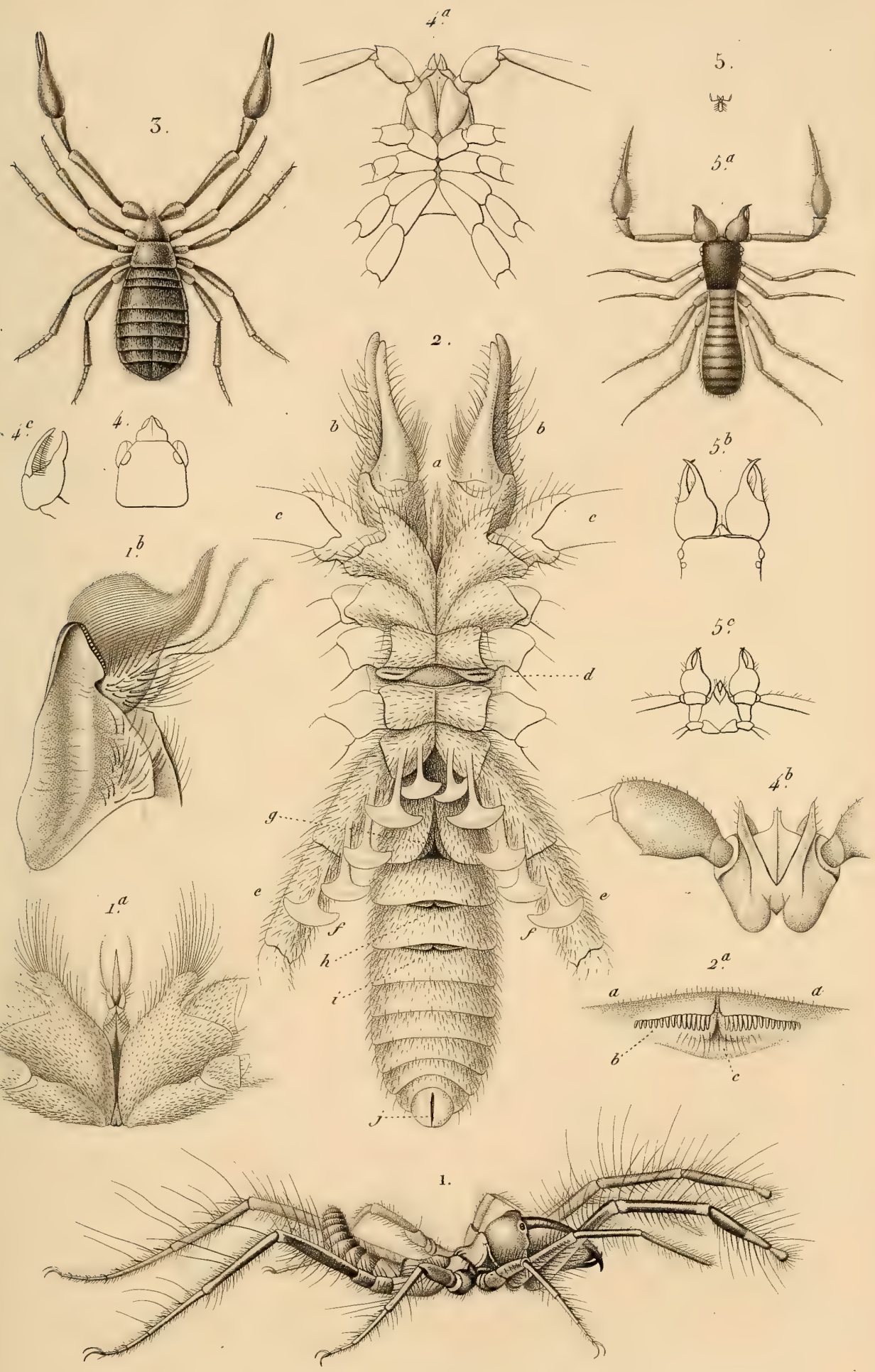

1. GALÉODE ARAIGNEE'. (Galeodes axaneoides.)

3. PINCE DE BRAVAIS. (Chelifer-Bravaisii.)

5. OBISIE TROMBIDIOIDE. (Obisium trombidioides.) 




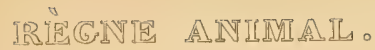
Qracbuides.

P1: 22.

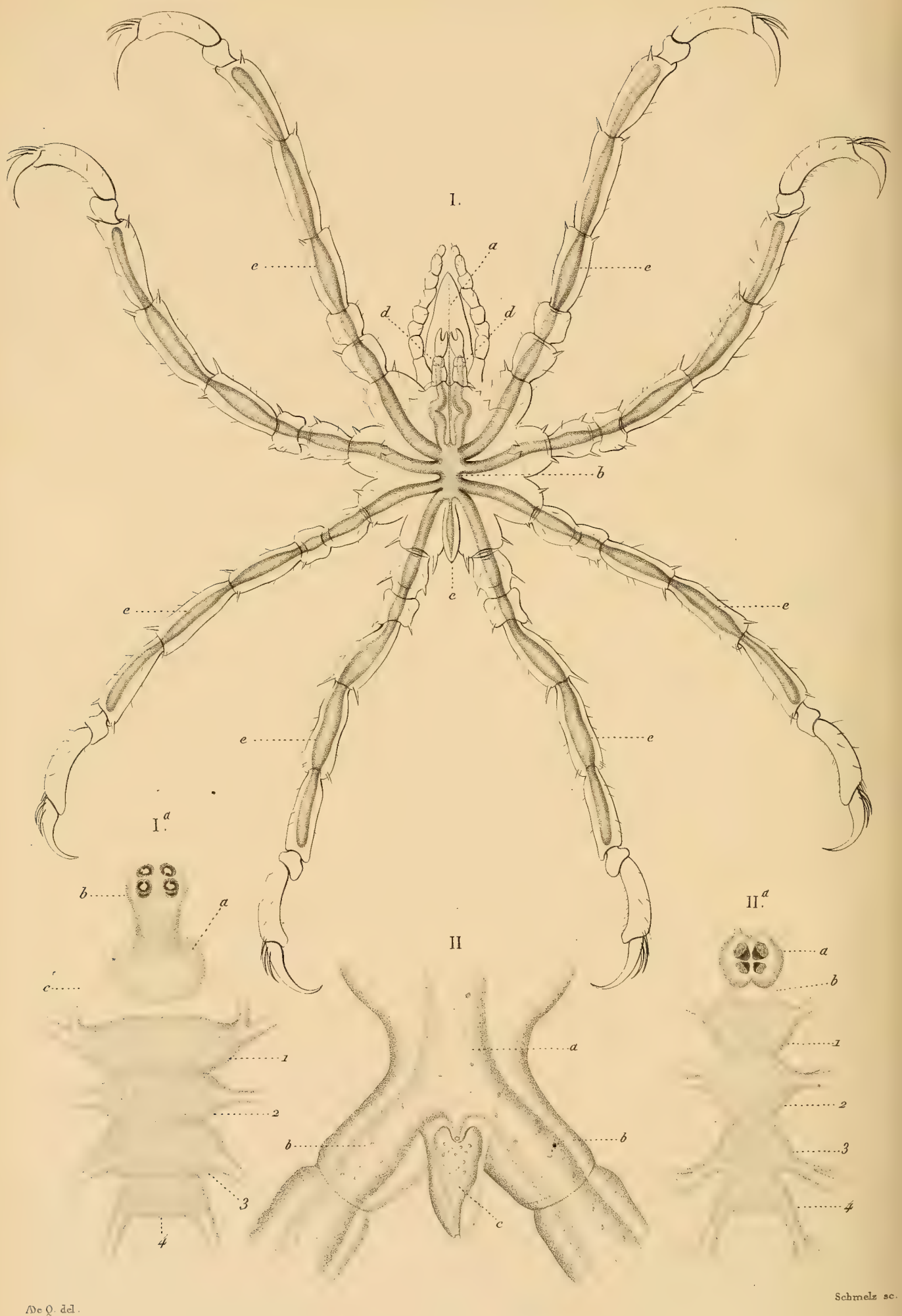

ORGANISATION DES PYCNOGONIDES

N. Remond unp. 


\title{
ARACHNIDES TRACHÉENNES.
}

\author{
PYCNOGONIDES.
}

Fig. 1. АммотнÉe PyGnogonö̈De. (Ammothea pyonogonozides. A. de A.) Grossie et montrant son appareil digestif injecté.

$a$. OEsophage. - $b$. Intestin. - $c$. Cloaque. - $d d$. Cœecum gastro-vasculaires pénétrant dans les antennes piuces. - eee. Cœcum gastro-vasculaires qui pénètrent dans les pattes jusqu'à l'antépénultième article exclusivement.

Fig. 1 a. Système nerveux de la même espèce.

\footnotetext{
a. Cerveau. - b. Ganglion ophthalmique. $-c$. Bandelette asophagienne dont je n'ai pu distinguer que les origines postérieures. - 1, 2, 3, 4. Ganglions abdomina ux.
}

Fig. 2. Partie postérieure du corps et du tube digestif du Phoxichile épineux (Phoxichilus spinipes. Latreille).

a. Extrémité postérieure de l'intestin donnaut naissance aux deux derniers cœcuin gastro-vasculaires $b, b,-c$. Cloaque.

Fig. $2 a$. Système nerveux de la même espèce.

a. Cerveau. - b. Bandelette œsophagienne.- 1, 2, 3. 4. Ganglions ab lominaux.

Ces dessins sont extraits d'un mémoire de M. de Quatrefages, inédit et relatif à l'anatomie des Pycnogonides. 




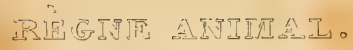

Aracbinides. P1.2I.
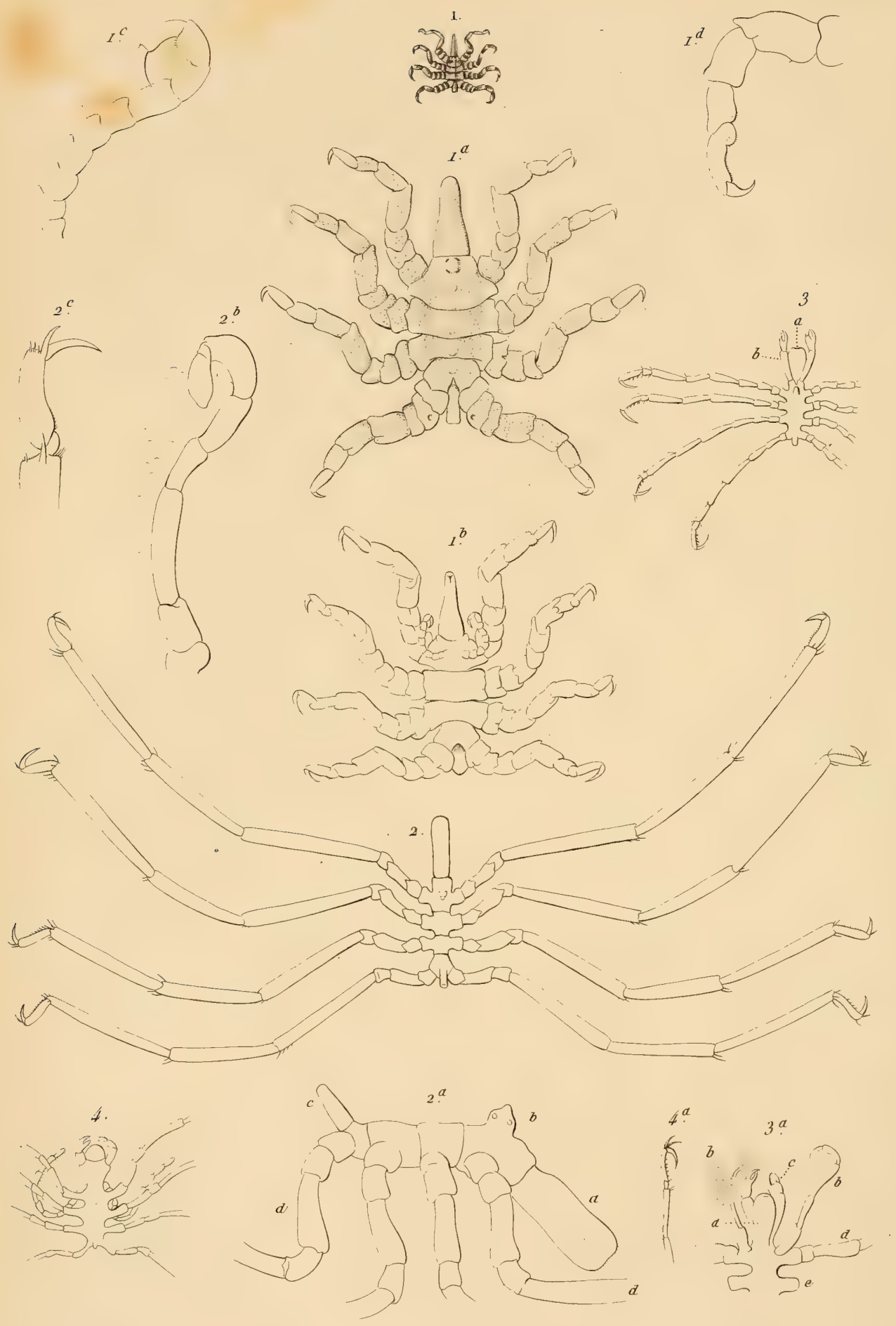

E.

Victor so:

1. PYCNOGONON LITTORAL. (Pycnogonum littorale)

2. PHOXICHIL E' EINEUX. (Phoxichilus spinosus.)

N. Rimond imp. 


\title{
ARACHNIDES TRACHÉENNES.
}

\author{
PYCNOGONIDES.
}

Genre PYCNOGONON. Pycnogonum. Brunnich, Muller, Fabricius, Latreille.

Fig. 1. Pycrognow Litroral. Pygnogonum littorale. Muller. De grandeur naturelle, male.

Fig. 1 a. Le même grố

Fig. 1 b. Une femelle en dessous, montrant les fausses pattes ovifères

Fig. 1 c. Fausse patte ovifère de la même, grossie.

Fig. 1 d. Une des pattes thoraciques.

Genre PHOXICHILE. Phoxichilus. Latr.

Fig. 2. Phoxichile épineux. Phoxichilus spinosus. Latr. Phalangium spinosum. Montagu. Individu male, grossi du double et vu en dessus.

Fig. $2 a$. Corps du même, vu de profil.

$a$. Tête. $-b$. Tubercule oculifère. - $c$. Abdomen. $-d, d$. Base des pattes.

Fig 2 b. Fausse patte ovifère de la femelle.

Fig. 2 c. Extrémité de l'une des pattes proprement diles.

Fig. 4. Phoxichile pieds-ÉpIneux. Phoxichilus spinipes. Latr. Gallene brevirostris. Johnston.

Le genre Gallène de M. Johnston établit le passage entre les précédens et les nymphons. Les pattes n'ont pas été figurées dans toute leur longueur.

Fig. $4 a$. Extrémité d'une patte.

Genre NYMPHON. Nymphon. Fabr.

Fig. 3. NyMphon ÉCARLate. Nymphon coceinentm. Johnston. Orythin coccinea. Ejusd. Phoxichilidium coccineum. Edw. Male de grandeur naturelle.

$a$. Tête - b. Palpes.

Le genre Orythia de M. Johnston ou Phoxichilidium de M. Milne Edwards établit le passage entre les Nymphons proprement dits et les Phoxichiles (Voyez Hist. Nat. des Crustacés, T. III. p. 535).

Fig. 3 $\alpha$. Extrémité antérieure du corps d'un individu femelle.

$a$. Tête. $-b$. Fausses-pattes ovifères, - $c$. Antennes-pinces (Latr.) déponrvues de paipes. - $d$. Base des pattes-antérieures. $-e$. Base des pattes de la seconde pairc.

Les fig. 3 et 4 sont copiées du mémoire de M. Johnsion; les autres ont été faites d'après nature. 




\title{
ARACHNIDES TRACHÉENNES.
}

\author{
HOL ĖTRES.
}

\section{TRIBU DES PHALANGIENS.}

\section{Genre FAUChEUR. Phalangium. Lin.}

Fig. 1. Faucheur des murallles. Phalangium cornutum. Lin. Ph. parietinum. De Geer. Individu mâle, de grandeur naturelle.

Fig. 1 a. Individu femelle (P. opilio. Lin.). Grossi au double.

Fig. 1 b. Céphalothorax du mème, vu en dessus et grossi davantage.

a. Céphalothorax. $-b$. Yeux, $-c$. Antennes-pinces. $-d$. Palpes. - e. e. Base des pattes.

Fig. 1 c. Individu mâle, vu de profil pour montrer les chélicères et l'organe de la génération.

a. Yeux. - b. Base des palpes. $-c$. Chélicères. $-d$. Appareil buccal. - $-e$. Verge en érection. $-f$. Abdomen.

Fig. 1 d. Région buccale.

$a$. Base des chélicères. - $b$. Palpes dont l'article basilaire $(d)$ est conformé en manière de mandibule, $-c$. Base des pattes de la première paire. $-e$. Lobes maxillaires. - $f$. Appendices situés à la base des pattes de la seconde paire, et paraissant représenter les secondes mâchoires. - $g$. Sternum au-devant duquel se trouve l'orifice de la génération.

Fig. 1 e. Individu femelle, vu en dessous et représenté au moment où l'oviducte $(a)$ est en éreclion.

(Toutes les figures précédentes sont d'après nature).

Fig. 2. Portion antérieure du corps du Faucheur égyptien male, montrant la manière dont les chélicères sont reployés contre la bouche. D’après M. Sawgray (ainsi que les trois détails suivans).

Fig. 2 a. Palpe du même.

Fig. 3. Portion terminale de l'une des pattes du Faucher gobte.

Fig. 3 a. Extrémité d'un palpe du même. 

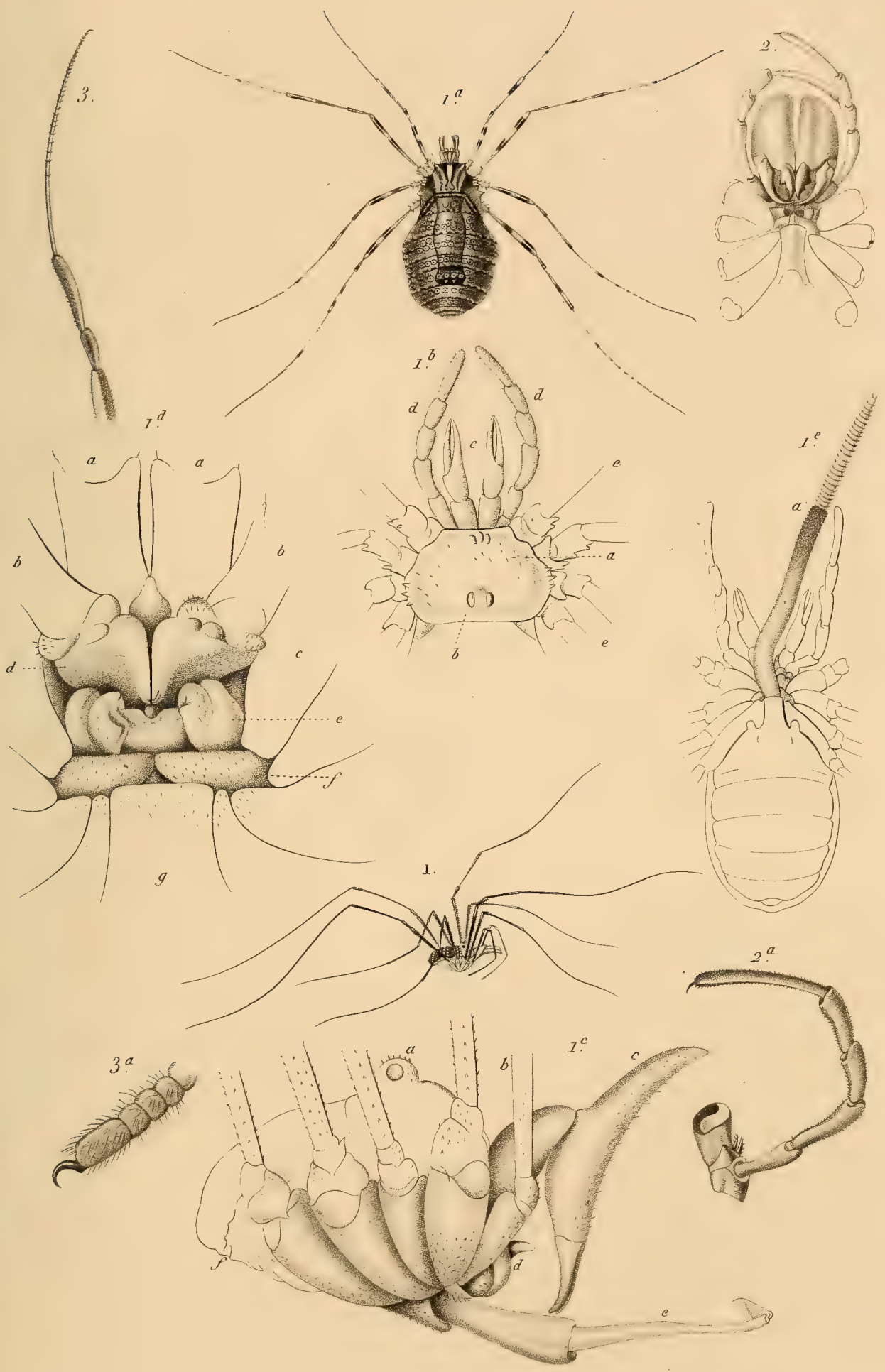

Victor:sc 




\title{
ARACHNIDES TRACHÉENNES.
}

\author{
HOLÈTRES.
}

TRIBU DES ACARIDES.

Genre MITE. Acarus. Lin.

DIVISION DES ACARIDES PROPRES.

S.-Genre TROMBIDION. Trombidium. Fabr.

Fig. 1. Trombidion colorant. Trombidium tinctorium. Fabr. Grossi environ trois fois.

Fig. 2. Trombidion du FAUGhedR. T. phalangii. Grossi.

Fiy. $2 a$. Croquis du même, vu en dessous.

Fig. 2 b. Un palpe.

Fig. 2 c. Mandibule.

Fig. 2 d. Larve du même, parasite du Faucheur des murailles.

S.-GENRE ERYTHRÉE. Erythrceus. Latr.

Fig. 3. ERYTHRÉE RURicale. Erythrous ruricola. Dugès. Beaucoup grossi.

Fig. 3 a. Palpe.

Fig. 3 b. Mandibule.

Fig. 3 c. Patte postérieure.

Fig. 4. Portion antérieure du corps de l'Erythrée ignipède.

Fig. $4 a$. Appareil digestif du même.

Fig. 4 b. Palpe de l'Erythrée isabelle.

Fig. $4 \mathrm{c}$. Mandibule de la même.

Fig. $4 d$. Palpe de l'Erythrée cornigère.

S.-Genre GAMASE. Gamasus. Latr.

Fig. 5. GAMASE DEs Coléoptères. Gamasus Coleoptrotorum. Lin. Vu en dessus el beaucoup grossi.

Fig. 5 $a$. Le mème vu en dessous.

Fig. 6. Gamase tétragonoïde. Gamasus tetragonoides, Grossi beaucoup.

Fig. 6 a. Extrémité d'un palpe.

Fig. 6 b. Mandibule.

Fig. 6 c. Son mordant mobile, grossi davantage.

Fig. 6 d. Extrémité d'une des pattes du même.

(La fig. 1 est d'après nature, les autres sont d'après les dessins originaux de Dugès accompagnant son travail sur les Acariens). 


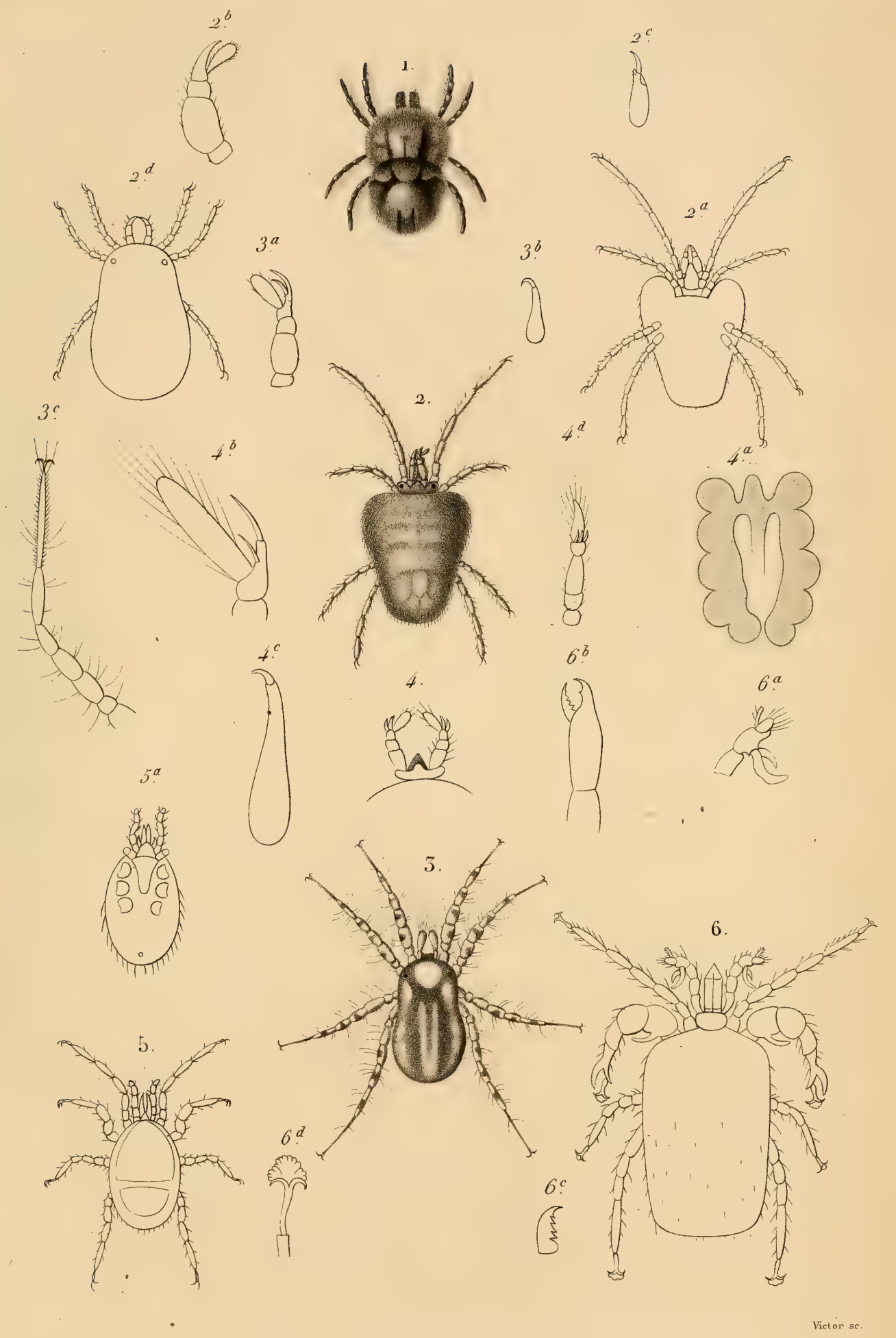

1.TROMBIDION COLORANT. (Trombidium tinctorium) 3. ERYTHRE'E RURICOLE. (Exythroeus ruricola. ) 2._. DU. KAUCHEUR. (__ _ Phalangii.) 5. GANASE DES COLÉOPTERES. (Gamasus colcoptratorum.) 6. GAMASE TETRAGONOIDE': (Gamasus tetragonoïdes.) 




\title{
TRACHÉENNES。
}

\author{
HOLETTRES.
}

Genre ACARIDES. Acarides. Latr.

Genre ORIBATE, Oribata. Latr.

Fig. 1. Oribate Caché. Oribata occulta; Pelops occultus. Koch. Grossi et vu en dessus.

Fig. $1 a$. Le même, de grandeur naturelle.

Fig. 1 b. Le même, vu en dessous et grossi.

Fiy. $1 c$. Céphalo-thorax vu en dessus.

Fig. 1 d. Méme partie vue en dessous.

Fig. 1 e. Pince mandibulaire.

Fig. $1 f$. Màchoire.

Fig. 2. ORibate Decumane. Oribata decumana; Hoplophora decumana. Koch. Grossi et vu en dessus.

Fig. $2 a$. Le même, de grandeur naturelle.

Fig. 2 b. Le même, contracté et vu de profil.

Fig. 2 c. Le même, vu en dessous.

Fig. $2 d$. La tête grossie davantage et vue en dessous.

Fig. 2 e. Mandibule, la pince étant fermée.

Fig. $2 f$. Pince ouverte.

Fig. 2 g. Mâchoire.

Fig. 3 h. Langue attachée à la face interne de la lèvre.

Fig. $2 i$. Tarse.

Genre SMARIDE. Smaridia. Latreille.

Fig. 3. Smaride papilleuse. Smaridia papillosa. Croquis du corps de cet acarien, d'après Dugès (loc. cit.).

Genre BDELLE. Bdella. Latreille.

Fig. 4. BDELle LARGE BEC. Bdella latirostris. Latr. Scirus latirostris. Herm. Grossi et vu en dessus.

Fig. $4 a$. Le même, de grandeur naturelle.

Fig. $4 b$. Céphalo-thorax vu en dessus et montrant la position des yeux.

Fig. $4 c$. Tête vue en dessous.

Fig. 5. Bdelle rarÉ. Bdella vestita. Koch.

Fig.5a. Grandeur naturelle.

Fig. 5 b. Céphalo-thorax vu en dessus.

Fig. 5 c. Extrémité du rostre.

Fig. 5 d. Mandibule.

Fig. 5 e. Extrémité de la mandibule. 


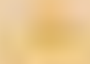

(1)

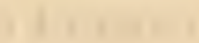
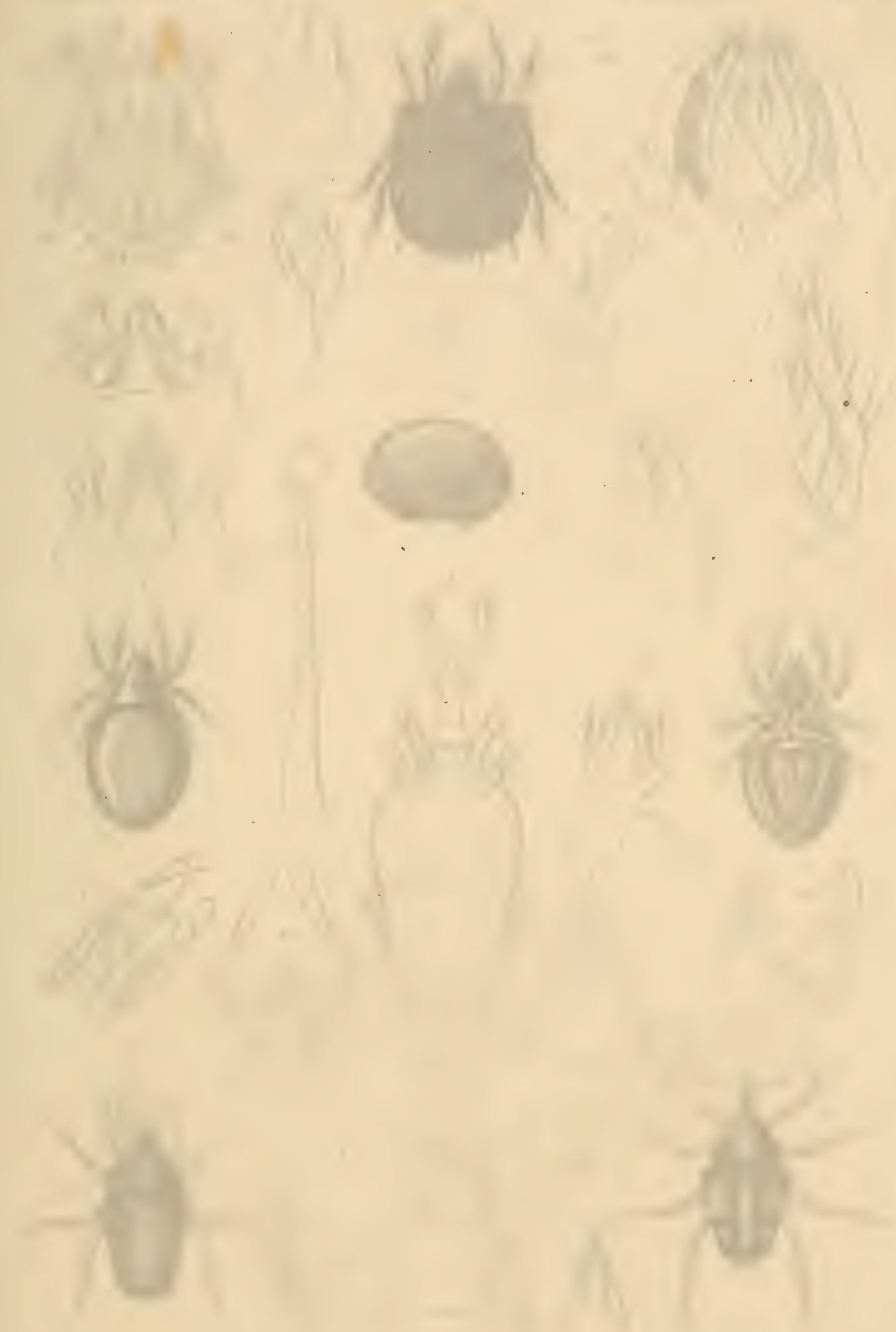


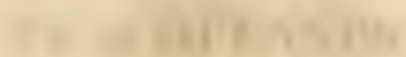

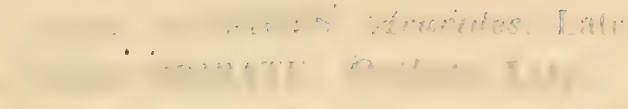

el. vis en? loḱstis.

Fig. 1a. Le méme, de srandeur ins

Fig. 1 b. I, meme, wi en desseris el giossi

Fig. 1 c. Géphato thoras vir en dessus.

fig. 1 d. Aldme partic racen dessoüs.

Fig. 1 e. Pince nuandibulate.

Fig. $x$ f. Hidchoire.

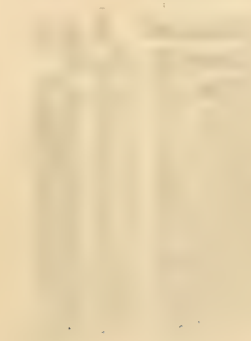

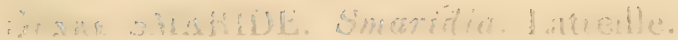

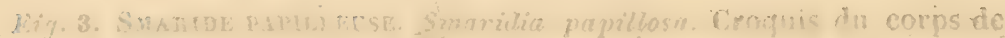

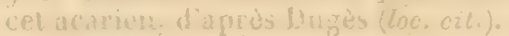

GRTE BDJiLle. Baclla. Latreille.

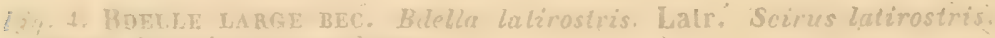
If im. Frossi et ru col dessus.

i.... 6.1. he mane de granden: naturelle.

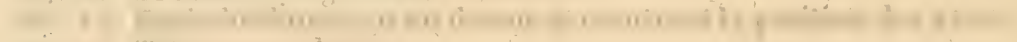

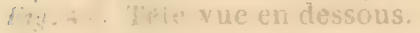

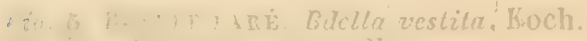

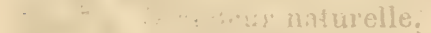

1. chorar vu en dessus.

i $\cdots$ i is

i. i. a... la manditule. 


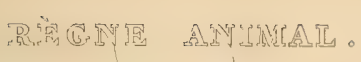

eAracbuides

P1. 25
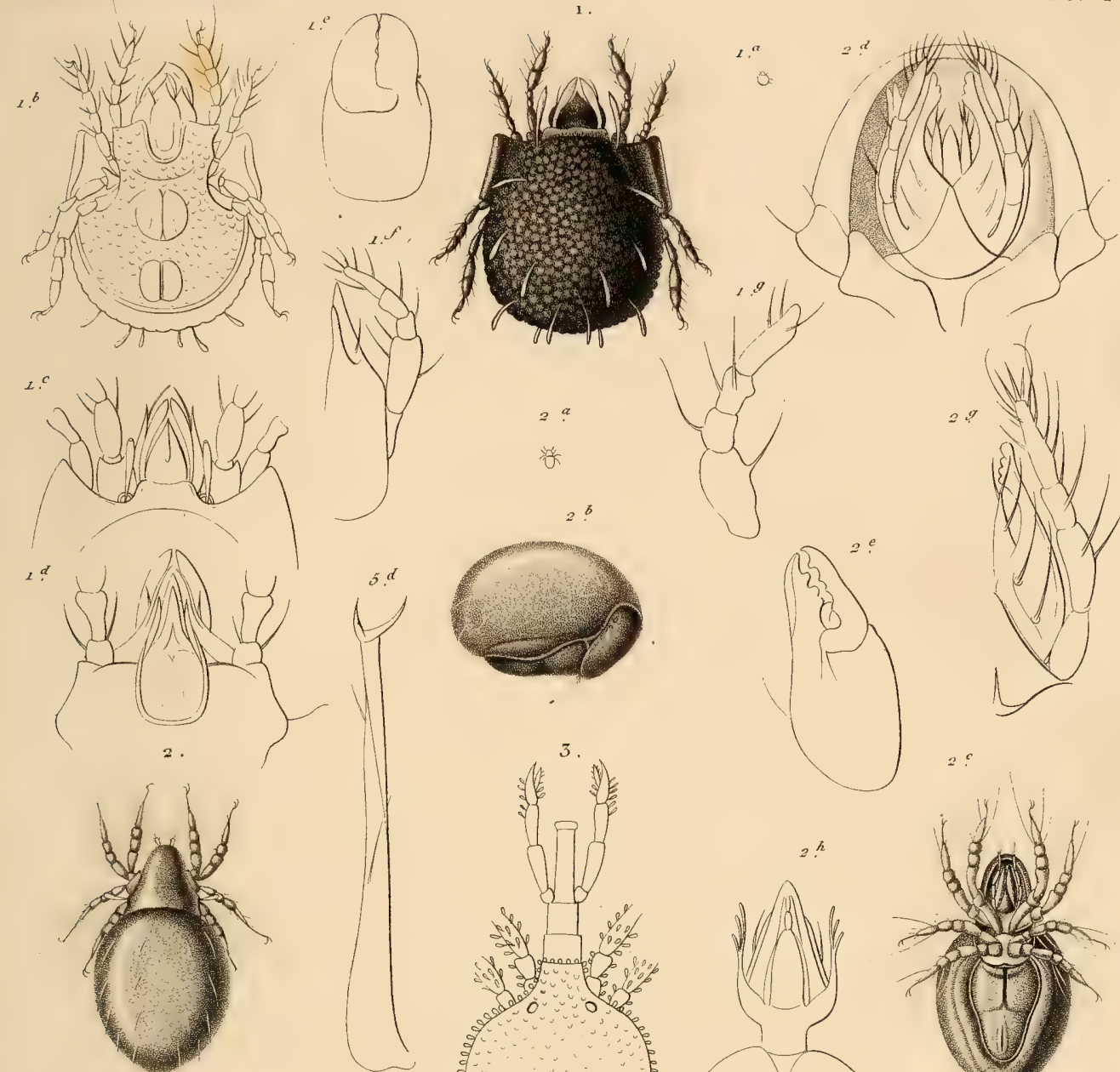

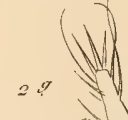
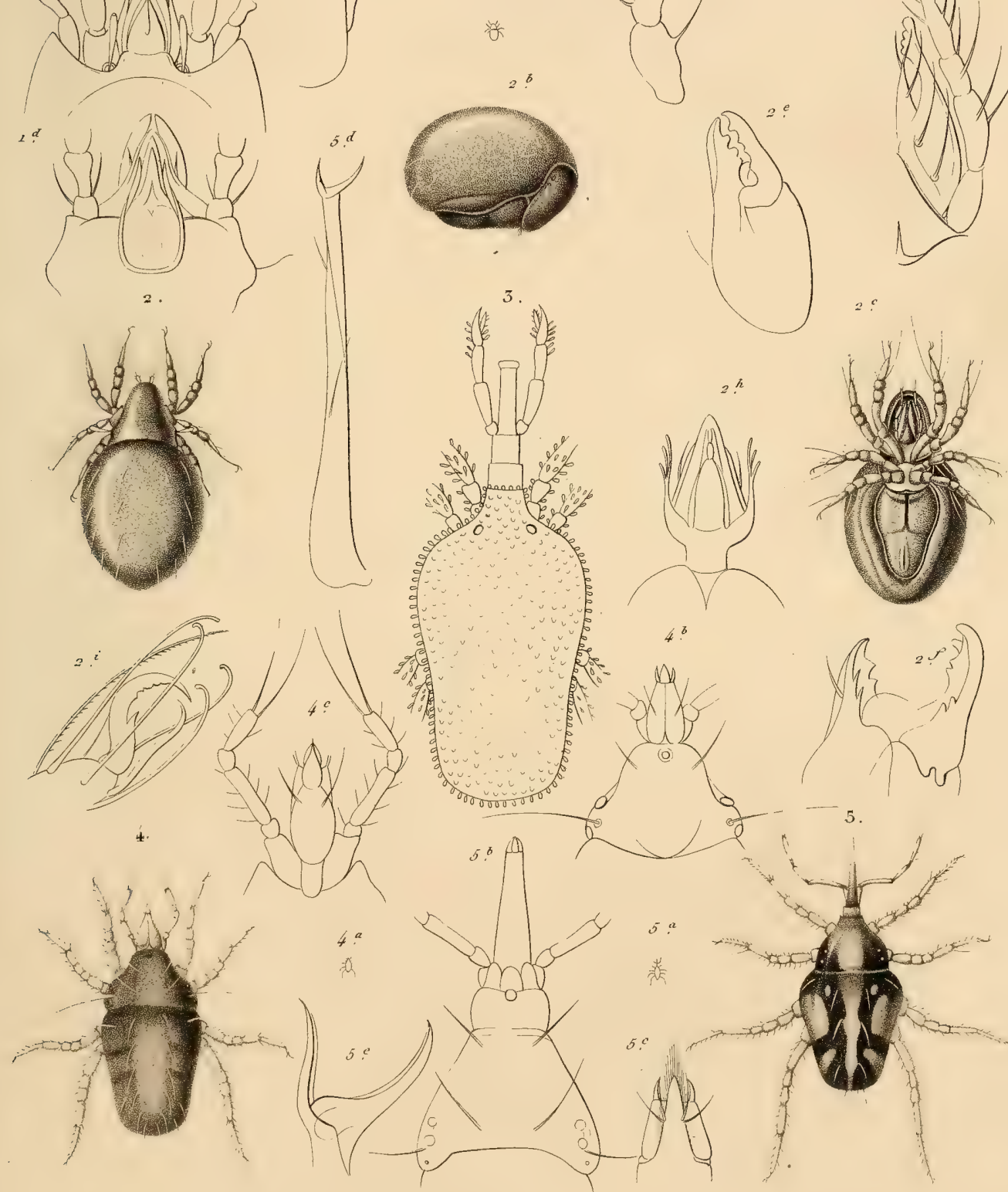

1. ORIBATE CACHE'

(0ribata occulta

2. ORIBATE DÉCUMANE (Oribata decumana)

3. SMARIDE PAPILLEUSE (Smaridia papillosa)

4. BDELLE LARGE BEC (Bdella latirostris) 




\title{
ARACHNIDES TRAGHÉENNES.
}

\author{
HOLĖTRES.
}

\section{TRIBU DES ACARIDES.}

Genre ACARE. Acarus. Fabr. Sarcoptes. Latreille.

Fig. 1. Acake de la galle du Cheval. Acarus equi. Saint-Didier. Sarcoptes equi. Hering. Psoroptes equi. Gervais. Femelle, vue en dessus et beaucoup grossie.

Fig. 1 b. La femelle, vue en dessous.

Fig. 1 c. Le mâle, vu en dessous.

Fig. $1 d$. La tête du même, grossie davantage et vue en dessous.

Fig. 1 e. Une des pinces.

Fig. $1 \mathrm{f}$. Une des pattes de la troisième paire du mâle.

Fig. $1 \mathrm{~g}$. Une des pattes de la troisième paire de la femelle.

Fig. 2. Acare nomestioue ou Ciron du fromage. Acarus domesticus. De Geer. Acarus siro. Hering. Tyroglyphus siro. Gervais. $\mathrm{Vu}$ en dessus et beaucoup grossi.

Fig. 2 a. Le mème, vu en dessous.

Fig. $2 b$. Jeune individu n'ayant encore que six pattes.

Fig. $2 c$. Tête du même, vue en dessous.

Fig. $2 d$. Extrémité d'une patte.

G ENRE UROPODE. Uropoda. Latreille.

Fig. 3. Croquis de l'Uropode véGétant. Uropoda vegetans. Latr. Grossi. Fig. 3 a. Une mandibule.

Fig. 3 l. Son extrémité.

Fig. $3 c$. Extrẻmité d'une patte du même.

(D'après nature, exceptées les fig. 3 à $3 c$ qui sont tirées du Mémoire de Dugès sur les Acariens, Ann. des $S_{\varepsilon}$. nat., $2^{\text {e }}$ série T. 2.) 


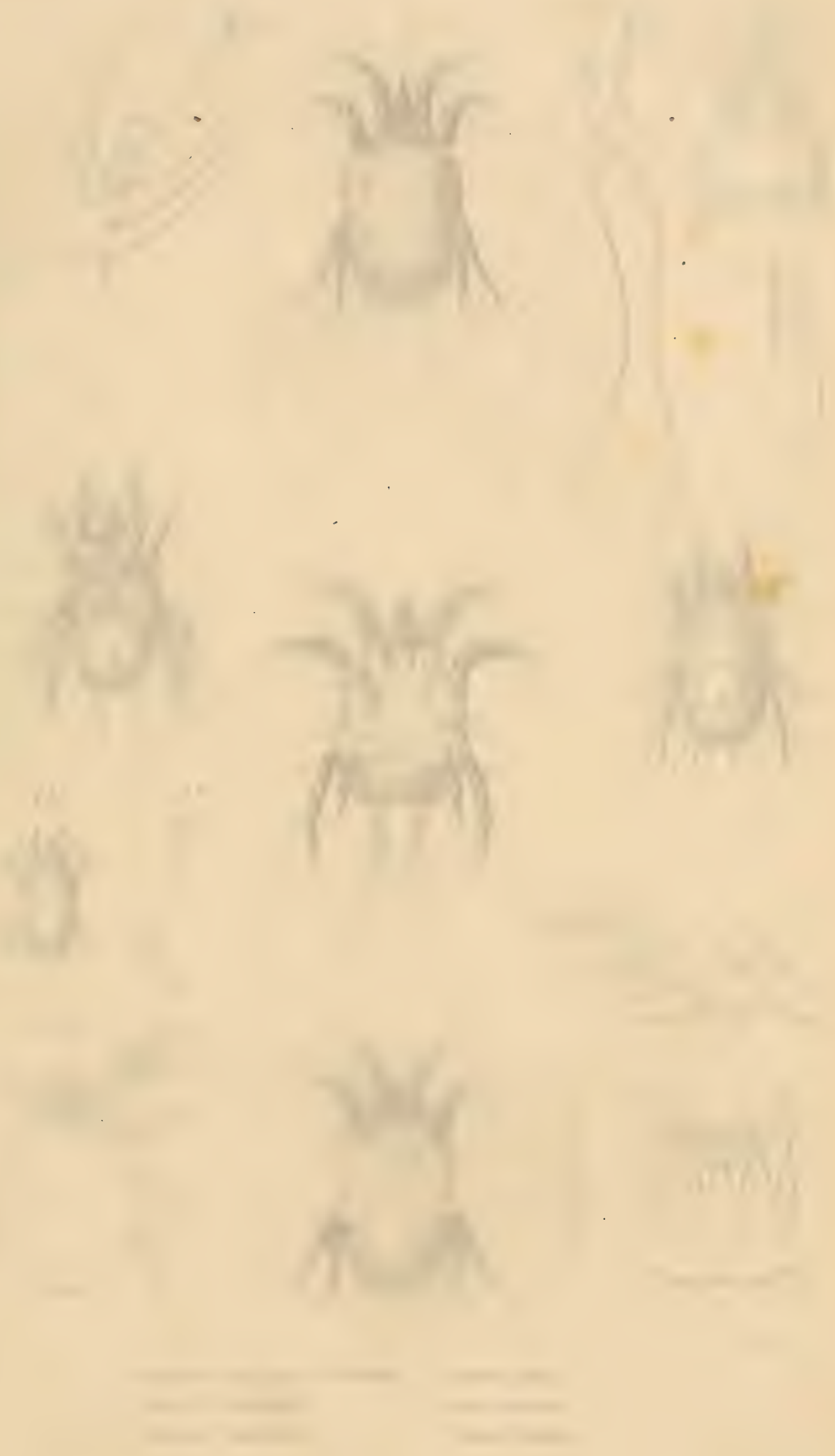




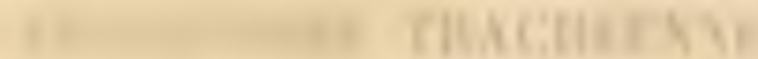

\section{Sint 1}

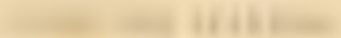

TENIE ACARE. Acarus. Fabr. Sareoptes Iatreille.

Fig. 1. Acame De La.gareg du cmeyal. Aonmes equi. Saint-Didier. Sarcoptes equi. Hering. Psoroptes equi. Gervais. Fermelle, vite on dessus eir beaucomp massic.

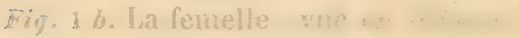

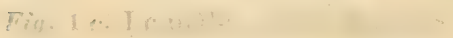

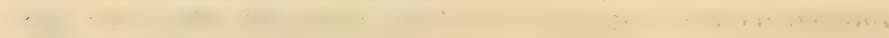

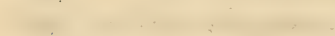

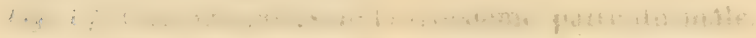

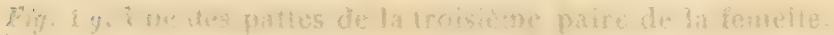

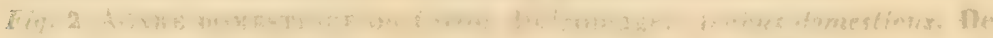

....

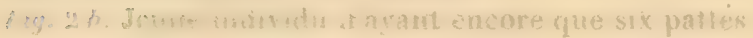

Fig. 2e. Tht du suene, vue en dessous:

Fig. 2s. Extrémaité d'une patte.

rimRE UTOPODE. Uropoda. Latreille.

Fig. 3. Cinquís de l'Uropode véglítant. Uropoda vegefnns. Latr. Grossi. Fiy. 3 u. The smantibulo.

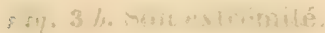

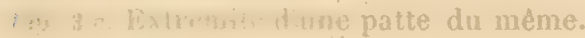

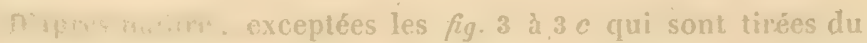

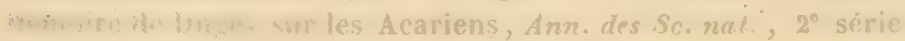




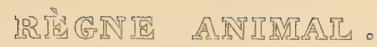

CAracbuides. Pl.26.

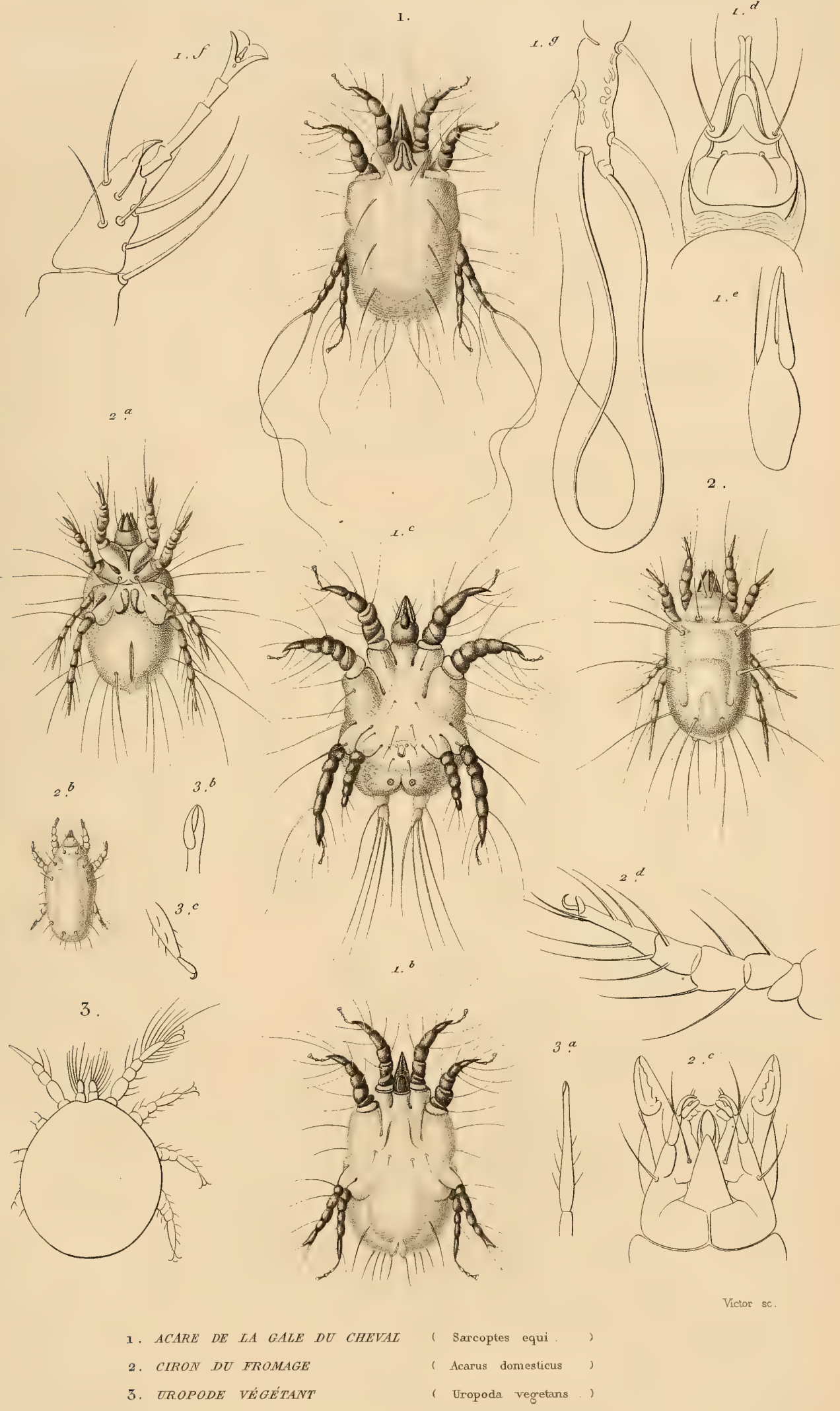






\section{TRACHÉENNES.}

HOLĖTRES.

Genne ACARIDES. Acarides. Latreille.

Genre IXODE. Ixodes. Latr.; Cynorcestus. Herm.

Fig. 1. Ixode DE GervaIs. Ixade Gervaisii. Lucas. Individu male, grossi et vu en dessus.

Fig. 1 a. Le même, de grandeur naturelle.

Fiy. 1 b. Le même, vu en dessous.

Fig. 1 ø. Appareil buccal du même.

Fig. 1 d. Une patte antérieure du mème.

Fiy. 1 e. Jeune femelle.

Fig. 1 f. La même, de grandeur naturelle.

Fig. $1 \mathrm{~g}$. Femelle adulte.

Fig. $1 \mathrm{~h}$. La même, de grandeur naturelle.

Fig. 1 . La même, vue en dessous.

Genre ARGaS. Argas. Latreille.

Fig. 2. Argas réfléGHi. Argas reflexus. Latr. Grossi et vu en dessus.

Fig. 3. Argas DE LA PIPISTRElle. Argas pipistrelle. Audouin. Individu jeune (Caris Vespertilionis? Latr.).

Fig. 3 a. Téte.

Fig. 3 b. Patte.

Genre ATACE. Atax. Fabricius ; Hydrachna. Müller.

Fig. 4. Atace tronqué. Atax truncatus. Koch.

Fig. $4 a$. Le mème, de grandeur naturelle.

Fig. 4 b. Région frontale et yeux.

Fig. $4 c$. Extrẻmité de l'abdomen vu en dessus.

Fig. 4d. Mème partie vue en dessous.

Fig. $4 \boldsymbol{e}$. Un des stigmates.

Genre LEPTE. Leptus. Latr.

Fig. 5. LePte AUtonnaL. Leptus automnalis, Latr. Grossi.

Fig. 5 a. Le même, de grandeur naturelle.

Fig. $5 \not b$. Appareil buccal vu en dessus.

Fig. 5 c. Le même, vu en dessous.

Fig. 5 d. Céphalo-thorax et yeux.

Fig. 5 e. Tarse.

(D'aprés nature.) 


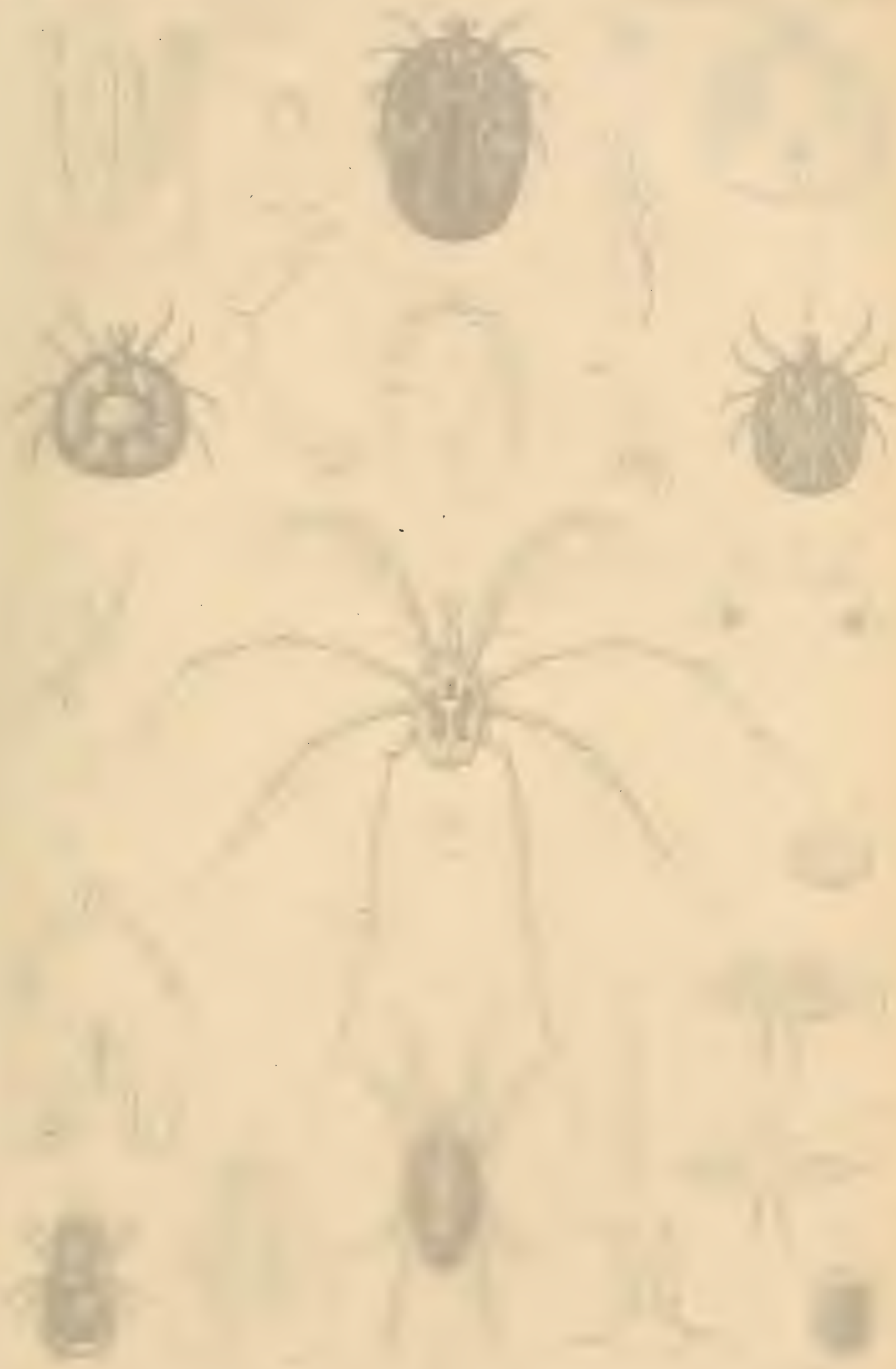

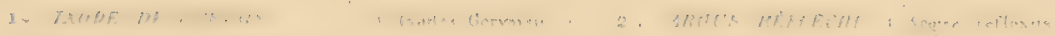

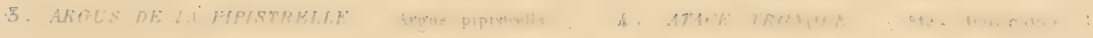




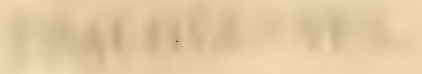

\section{a.........111.}

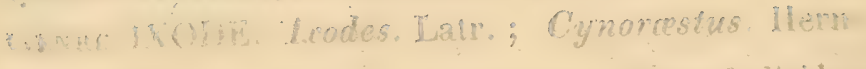

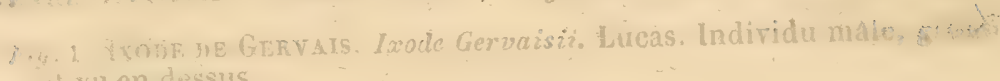

t vu en dessus.

Fiv. 1 a. Le metme, de grandeur naturelle.

Fin. 1b. Le meme, va en dessons.

Fio. 1 c. Apparail buccal du méne.

Fig. ¿ đa. Une valte antérieure du mème.

- Fin. 1e Jeune lemelle.

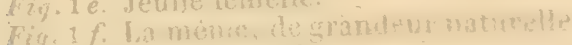

tig ig. Femelle adelte

Pig. 1h. Wa beme di

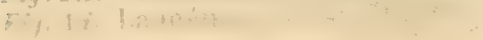

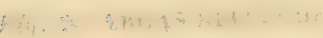

$\therefore$

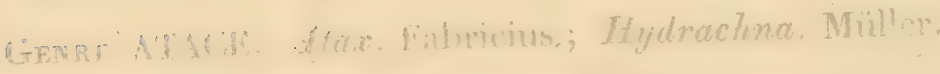

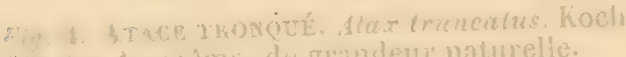

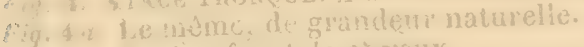

Fin. 3. Hétion fiontale et ymix.

Fir. ar. Extrénité ate l'abdumen vu en dessus,

Fin fat. Menze parlic vue en dessous.

Fiq. ie rin les stignates.

1.FPR: 1.HITL, Lentus. ILatr.

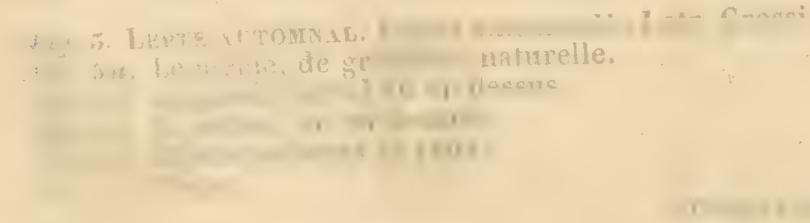


RTIG

Aracbuider. P1. 27.

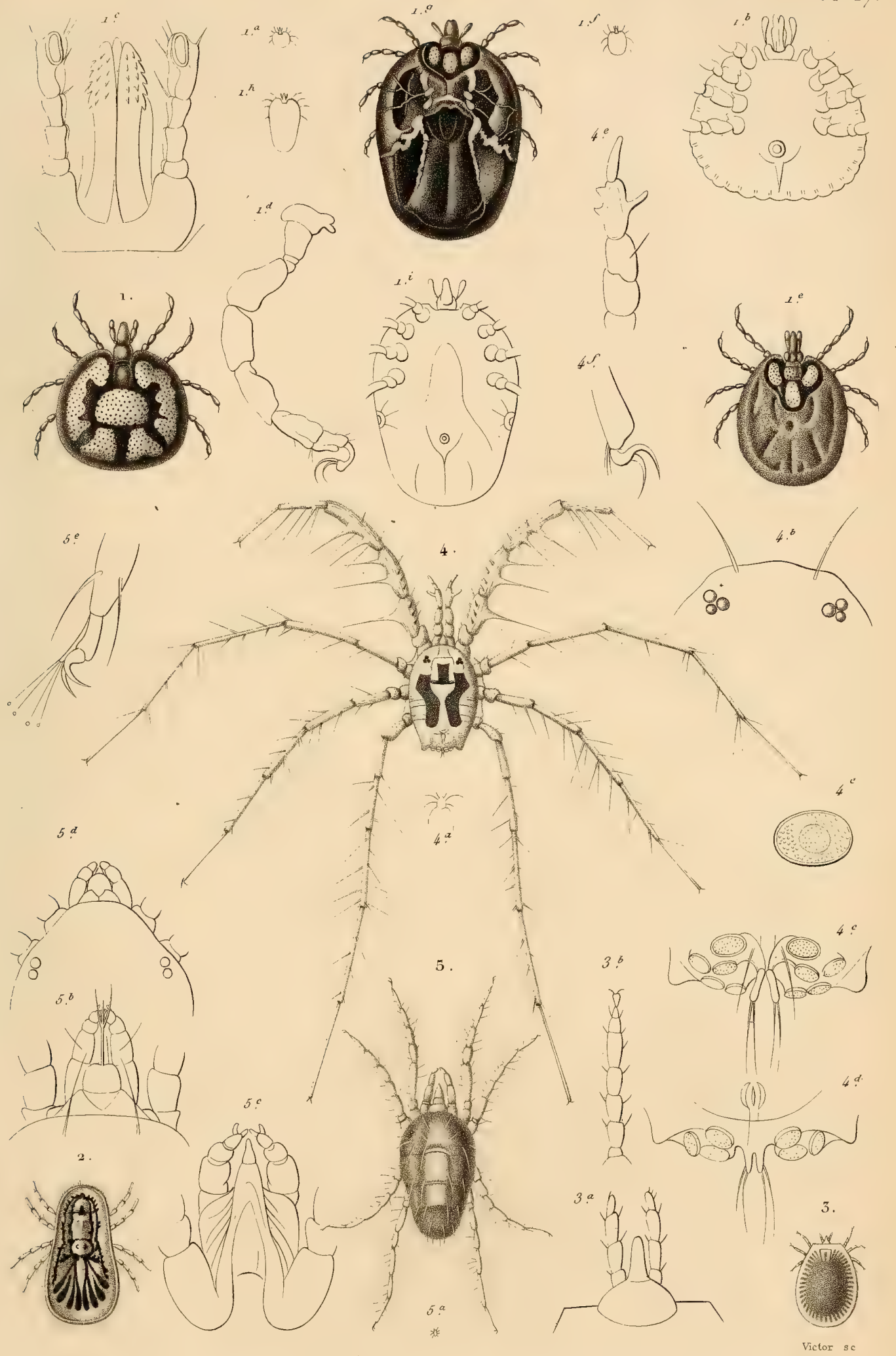

1. IXODE DE GERVAIS (Ixodes Gervaisii) 2. ARGUS RÉELÉCHI (Ágas reflexus.)

3. ARGUS DE LA PIPISTRELIE (Argas pipistrelle ) 4. ATACE TRONQUÉ (Atax truncatus.)

5. LEPTE AUTOMNAL ( Leptus autumnalis) 




\section{ARACHNIDES TRACHÉENNES.}

\section{HOLËTRES.}

Gen me MITE. Acarus. Lin.

Section des HYDRACHNELLES. Hydrachnelloe. Latr.

S.-GenRe HYDRACHNE, Hydrachna. Latr.

Fig. 1. HYDRACHNE GÉOGRAPHIQUE. Hydrachna geographica. Muller. Grossi (d'après Hahn).

Fig. 1 a. Croquis du méme, de grandeur naturelle.

Fig. 2. Hydrachne Globule. Hydrachna glohulus. Hermann. Grossie et vue en dessous (d'après Dugès).

Fig. 2 u. Bec de la même, représenté de profil et garni d'un de ses palpes.

Fig. $2 b$. Mandibules, très grossies.

Fig. $2 c$. Extrémité d'un des pieds.

Fig. $2 d$. Larve de la même récemment éclose et beaucoup grossie.

Fig. 2 e. Un de ses palpes.

Fig. $2 f$. Partie postérieure du corps d'une Nèpe cendrée grossie et portant un grand nombre de nymphes d'hydrachnes à différens degrés d'accroissement (ce sont ces parasites qui ont été décrits par Audouin sous le nom d'Aclysies; voyez le texte de Latreille.

Fig. $2 \mathrm{~g}$. Trois de ces nymphes détachées.

Fiy. $2 h$. Une de ces nymphes dont le développement est plus avancé ; on a indiqué au trait l'accroissement qu'elle aurait prise en avançant en âge.

Fig. $2 i$. Une nymphe de l'àge de celles représentées fig. $2 g$, grossie davantage et vue en dessous pour montrer :

$a$. Le suçoir en forme de bec ou de tête, et portant deux palpes. - b. Deux des anciennes pattes de la larve, réduites à leur portion épidermique; les quatre autres sont tombées. $-c$. Les moignons qui constituent les rudimens des pattes et des palpes de l'animal parfait. $-d$. Une tache médiane qui se rapporte aux organes de la génération.

Fig. $2 k$. Une nymphe à son dernier degré d'accroissement et vue de profil; on a rendu les tégumens transparens pour faire voir la jeune Hydrachne près d'éclore.

Fig. 3. HXDRACHNE ARLEQUIN. Hydrachna histrionica. Atace arlequin. Dugès. De grandeur naturelle.

Fig. $3 a$. La même, grossie.

Fig. 3 b. Portion antérieure du corps, vue en dessous pour montrer la disposition de la lèvre, les hanches et l'ouverture des organes génitaux.

Fig. 3 c. Un des palpes.

Fig. 3 d. Une des mandibules.

S.-Genre EYLAIDE. Eylais. Latreille.

Fig. 4. Eylaïde Étendeuse. Eylais extendens. De grandeur naturelle.

Fig. 4 a. La mème, grossie.

Fig. 4 b. Le corps de la mème, vu en dessous, montrant la bouche et les palpes $(a)$; les hanches réunies en deux groupes de chaque côlé; l'ouverture génitale $(b)$; l'anus $(c)$ et les quatre stigmates.

Fig. $4 \mathrm{c}$. La bouche, très grossie, avec son capuchon à la base des palpes.

Fig. $4 d$. Un des palpes, très grossi.

Fig. 4 e. Dernier article, plus grossi.

Fig. $4 \mathrm{f}$. Mandibule dont le crochet semble replié.

Fig. $4 \mathrm{~g}$. Le bout de la mandibule avec le crochet redressé.

Fig. $4 h$. Larve de l'Eylaïde élendeuse nouvellement éclose.

(D'après les dessins faits par Dugès pour accompagner son mémoire sur les Acariens, publié dans les Annales des seiences naturelles, $2^{\mathrm{e}}$ série, $\mathrm{t}$. I). 


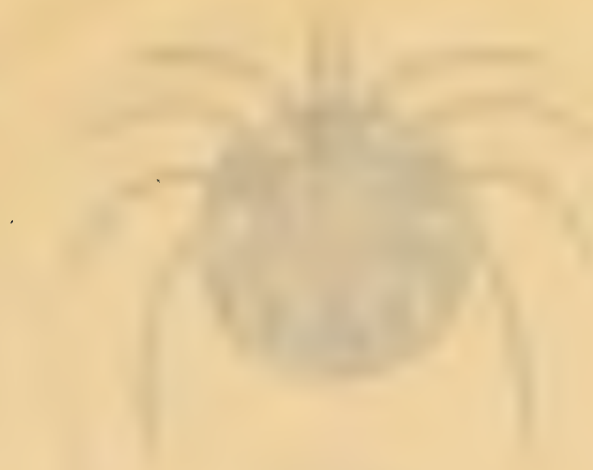

. 



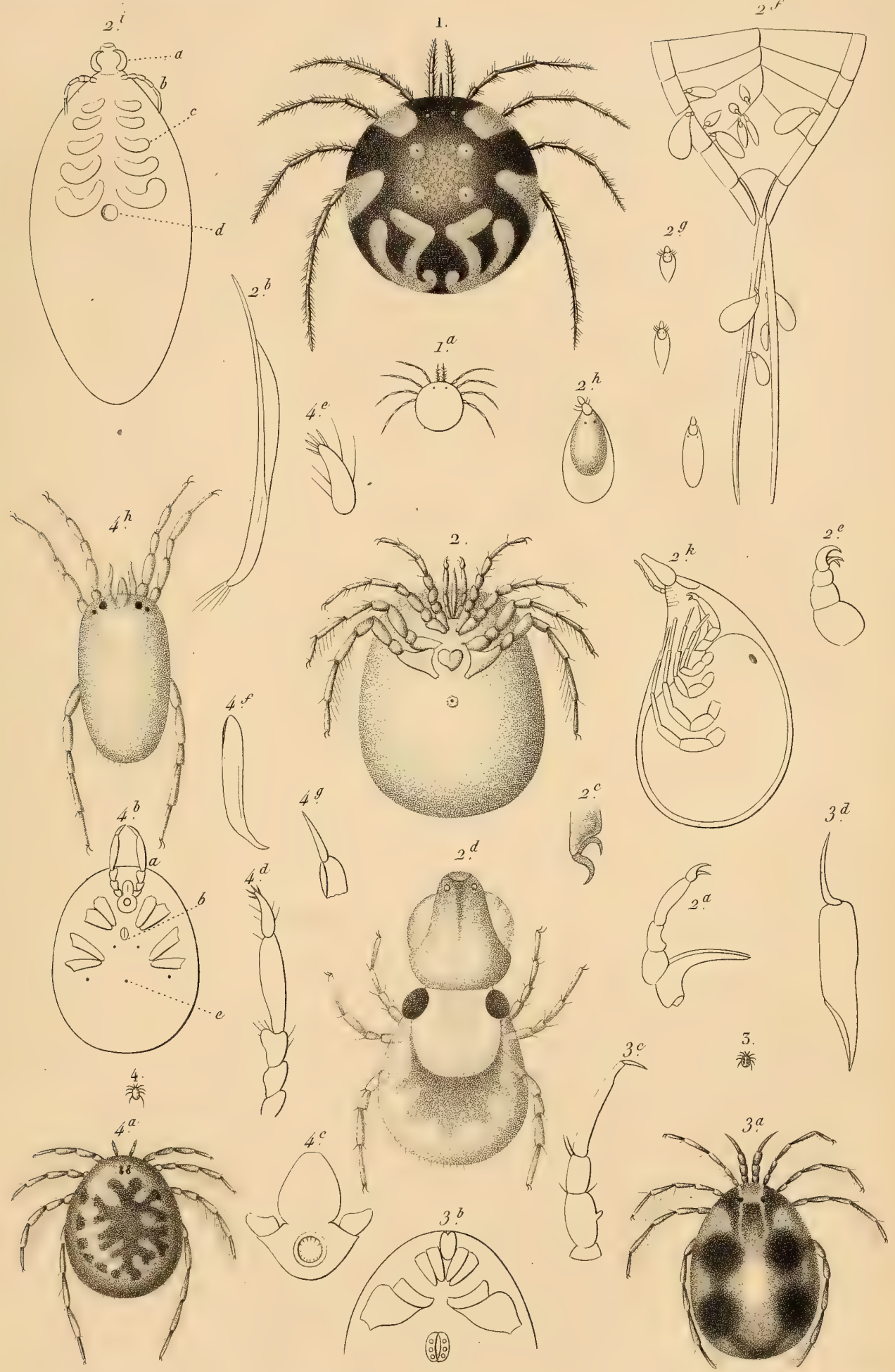

Victor sc.

1. HIDIRACHNE GEOOGRAPHIQUE. (Hydrachna greographica.) 


1 

